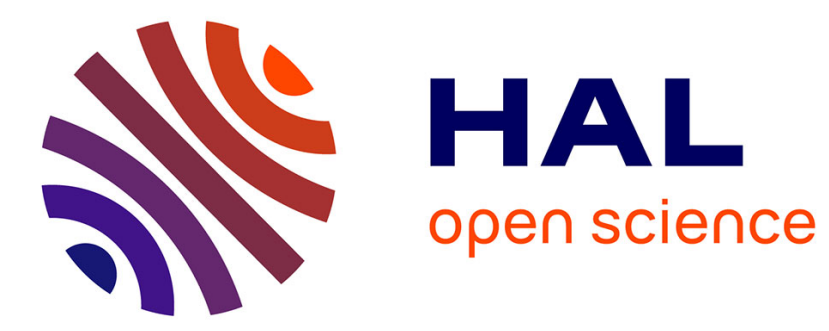

\title{
Le site de La Roquette à Tresques (Gard) et le Néolithique final du bassin bas rhodanien
}

\author{
Catherine Georjon, Vianney Forest, André Raux
}

\section{To cite this version:}

Catherine Georjon, Vianney Forest, André Raux. Le site de La Roquette à Tresques (Gard) et le Néolithique final du bassin bas rhodanien. Gallia Préhistoire - Archéologie de la France préhistorique, 1999, 41, pp.253-297. 10.3406/galip.1999.2166 . hal-01957002

\section{HAL Id: hal-01957002 \\ https://hal.science/hal-01957002}

Submitted on 17 Dec 2018

HAL is a multi-disciplinary open access archive for the deposit and dissemination of scientific research documents, whether they are published or not. The documents may come from teaching and research institutions in France or abroad, or from public or private research centers.
L'archive ouverte pluridisciplinaire HAL, est destinée au dépôt et à la diffusion de documents scientifiques de niveau recherche, publiés ou non, émanant des établissements d'enseignement et de recherche français ou étrangers, des laboratoires publics ou privés.

\section{(ㅇ)(1) $\$$}

Distributed under a Creative Commons Attribution - NonCommercial - NoDerivatives $\mid 4.0$ 


\section{Le site de La Roquette à Tresques (Gard) et le Néolithique final} du bassin bas rhodanien

Cathy Georjon, Vianney Forest, André Raux

\section{Citer ce document / Cite this document :}

Georjon Cathy, Forest Vianney, Raux André. Le site de La Roquette à Tresques (Gard) et le Néolithique final du bassin bas rhodanien. In: Gallia préhistoire, tome 41, 1999. pp. 253-297;

doi : https://doi.org/10.3406/galip.1999.2166

https://www.persee.fr/doc/galip_0016-4127_1999_num_41_1_2166

Fichier pdf généré le 19/07/2018 


\title{
Résumé
}

Le gisement de La Roquette, à Tresques, est situé dans la vallée de la Tave, petit affluent du Rhône au nord du département du Gard. Trois occupations principales ont été identifiées : un dépôt du Néolithique ancien final ou du Chasséen ancien, un probable four attribuable au Chasséen classique et un habitat du Néolithique final. Cette dernière période est la mieux documentée : un sol induré, des fosses et des calages ont été mis en évidence. Le mobilier, abondant, pose des problèmes d'attribution chronoculturelle. Trois hypothèses sont examinées ici. La première $s$ 'appuie sur des comparaisons avec les séries de transition Néolithique moyen-final. La deuxième permet de réexaminer les arguments retenus pour identifier le passage du groupe de Ferrières au groupe de Fontbouisse. Enfin, la dernière proposition prend en compte des rapprochements typologiques avec les faciès rhodaniens et provençaux de la fin du Néolithique.

\begin{abstract}
The Neolithic site la Roquette (Tresques) is located in the Tave valley, minor tributary of the Rhône, north of the Gard. Three main occupations have been identified : an Early Neolithic or Early Chassean sacred trust, a likely classical Chassean baker's oven and a Later Neolithic dwelling. This period turned out to be the most obvious one : hard earth, pits and wedgings have been unveiled. The plentiful archaeological stock makes the chronocultural attribution difficult. Three hypothesis are here developed. The first one, the chronological side, proposes an early dating in the Later Neolithic Age, in comparison with the assemblages of the Middle and Later Neolithic Age transitional period. The second one deals with the arguments considered to identify the passage of the Ferrières group to the group of Fontbouisse. The last one is based on typological comparisons with the rhodanian and Provence fades at the end of the Neolithic.
\end{abstract}




\title{
LE SITE DE LA RoQUeTTE À TRESQUES (GARD) ET LE NÉOLITHIQUE FINAL DU BASSIN BAS RHODANIEN
}

\author{
Cathy GEORJON* avec la collaboration de Vianney FOREST ${ }^{* *}$ et André RALX****
}

Mots-clés. Gard, Tresques, La Roquetté, Lamguedor, Provence, Néolithique ancien, Néolithique moyen, Néolithique final, habitat, dépôt
céramique, structures de combustion, sol aménagé, fosses, calages, chronotypologié.

Key-words. Gard, Tresques, La Roquette, Languedor, Provence, Early Neolithic, Middle Neolithic, Late Neolithic, duelling, sacred trust, hearthes, hard earth, pits, post hole stones, chronotypology.

Résumé. Le gisement de I.a Roquette, à Tresques, est situé dans la vallée de la Tatue, petit affluent du Rhône au nord du département du Gard. Trois occupations principales ont été identifiées : un dépôt du Néolithique ancien final ou du Chasséen ancien, un probable four attribuable au Chasséen classique et un habitat du. Néolithique final. Celle dernière période est la mieux documentée : un sol induré, des fosses et des calages ont été mis en ínidence. Ie mobilier; abondant, pose des problèmes d'attribution chronoculturelle. Trois hypothèses sont examinées ici. La première s'appuie sur des comparaisons aver les séries de transition Néolithique moyen-final. La deuxième permet de réexaminer les arguments retenus pour identifier le passage du groupe de Ferrières au groupe de Fontbouisse. Linfin, la dernière proposition prend en compte des rapprochements lypologiques aver les faciès rhodaniens et provensaux de la fin du. Véolithique.

\begin{abstract}
The Neolithic site la Roquette (Tiesques) is located in the Tave valley, minor tributary of the Rhone, north of the (iard. Three main occupations haze been identified : an Early Neolithic or Early Chassean sacred trust, a likely classical chassean baker's oven and a Later Neolithic duelling. This period turned out to be the most obvious one : hard earth, pits and wedgings have been unveiled. The plentiful archaeological stock makes the chromocultural attribution difficult. Three hypothesis are here developed. The first one, the chronological side, proposes an earty dating in the Later Neolithic Age, in comparison with the assemblages of the Middle and Later Neolithic Age transitional period. The second one deals with the arguments considered to identify the passage of the Ferrières group to the group of Fontbouisse. The last one is based on typological comparisons with the rhodanian and Provence facies at the end of the veolithic.
\end{abstract}

L.e gisement de La Roquette a fait l'objet d'une fouille de sauretage urgent en 1994, sous la responsabilité d'André Raux avec le groupe archéologique de la vallée de la Tave'. L'occupation principale est attribuable au Néolithique final. Outre les nombreux aménagements observés sur $100 \mathrm{~m}^{2}$ seulement, l'intérêt de cette occupation est

\footnotetext{
* Doctorante, LMR 6636 du CNRS : "Économies. sociétés et enviromnement préhistoriques ". Aix-en-Provence, La Valsière, 8 place Beau-de-Rochas, F-3.1790 (irabels.

s: Docteur vétérinaire, archéozoologue, 196 allée des Colverts. F-34280 lat Grande--Motte.

*** I.c Colin, F-30330 Tresques.

1. Le présent article fait référence à un DEA soutenu à l'université de Provence Aix-Marseille 1 (Ceorjon, 1997). Nous remercions . Vichel Olive pour le traitement infographique des figures (laboratoire des arts graphiques du Service régional de l'archéologie de Provence).
} 
lié aux caractéristiques de l'abondant mobilier recueilli, céramique surtout. Celui-ci s'intègre difficilement dans le cadre chronoculturel postchasséen du Languedoc oriental. La situation du gisement, à la lisière rhodanienne de cette région, impose d'étendre le cadre des comparaisons à la Provence occidentale et au sud de la Drôme.

Par rapport au groupe de Ferrières, Ia Roquette se distingue essentiellement par l'indigence des décors céramiques. Cette divergence, associée à d'autres indices, pourrait s'expliquer par une position chronologique en amont du Ferrières constitué, ou bien, au contraire, dans une phase finale de ce groupe, mais encore, par la situation marginale du gisement par rapport à l'aire d'extension du Ferrières.

La plupart des ensembles attribućs au "Nćolithique récent " dans le Languedoc oriental sont situés à l'ouest de cette région, dans les garrigues montpelliéraines et aux abords des Causses. Ces ensembles, essentiellement caractérisés par le couple écuelles carénées de tradition chasséenne/jarres à cordons annonçant le Néolithique final, sont, pour la plupart, en marge de l'aire d'extension du Saint-Pono-Vérazien et des Treilles, groupes qui intègrent les formes carénées et les adaptent, tandis que le Ferrières les exclut totalement. Dans les garrigues gardoises et ardéchoises comme en Provence, quelques éléments dont la plupart ont été récemment découverts sur le tracé du TGV Méditerranée pourraient se rapporter à l'extrême fin du Chasséen ou au tout début du Néolithique final. Ils permettent une analyse du matériel de La Roquette par rapport à cet horizon chronologique.

Par ailleurs, quelques éléments ont récemment été analysés dans la perspective d'une transition entre le Ferrières et le Fontbouisse (Gutherz, Jallot, 1995). Là encore, l'indigence des décors nous incite à des rapprochements avec La Roquette.

Enfin, si les faciès du Ferrières et du Fontbouisse sont bicn définis (Guther\%, 1975, 1984 ; Guther», Jallot, 1995), il reste des zones d'ombre aux marges de l'aire d'extension de ces cultures. À l'est, dans le bassin du bas Rhône, des phénomènes de métissage culturel sont connus depuis longtemps en rive gauche du fleuve, notamment près de sa confluence avec la Durance (sites de La Balance, de la Banque de France, du Conservatoire, de la Place du Palais et de La Fontaine à Avignon, cf. Gagnière, Granier, 1972; Sauzade, 1983) et dans les Alpilles (Escanin 2 aux Baux-deProvence, $(f$. Montjardin, 1970). Tous les types de contacts sont envisageables entre deux contrées mitoyennes : cir- culation des idées, des objets ou des hommes, et peut-être même les trois en alternance sur la durée, ce qui complique l'analyse culturelle des groupes humains récepteurs. Les groupes de Fraischamp ct dc Rhônc-Ouvèze, qui se succèdent dans la Provence rhodanienne, montrent bien le caractère durable et renouvelé de ces contacts (D'Anna, 1995a et b). Ces groupes, qui s'inspirent très nettement du Ferrières et du Fontbouisse, adaptent les thèmes décoratifs de ces deux cultures en des compositions parfois spécifiques (Muller, D’Anna, 1986; Sauzade et al., 1990). Ces manifestations florissantes n'ont pour l'instant pas de relais satisfaisant de l'autre côté du Rhône où les sites ferrériens et fontbuxiens sont rares dans la plaine littorale du bas Rhône et les costières du Gard (Gutherz, 1984). La petite série du Mas de Miraman à Nîmes s'inscrit même à contre-courant de cette expansion des décors languedociens vers l'est (Roger, Gutherz, 1986). Elle est à l'origine de l'identification d'un faciès rhodanien périphérique dont la rareté des décors peut être due à des contacts avec les groupes provençaux (Gutherz, 1984; Gutherz, Jallot, 1995). Cette caractéristique (le manque de décors) est commune à plusieurs ensembles du Néolithique final-Chalcolithique situés dans la basse et moyenne vallée de l'Ardèche et dans le sud de la Drôme (style des Bruyères et style d'Allan). Le site de La Roquette peut être ajouté à la liste et permet de considérer quelques indices des relations avec la Provence un peu plus démonstratifs que l'indigence des décors céramiques.

\section{PRÉSENTATION DU SITE}

\section{LE CADRE GÉOGRAPHIQUE}

Ia commune de Tresques se situe dans la partie septentrionale du département du Gard. Elle est baignée par la Tave, petite rivière (environ $30 \mathrm{~km}$ de long) qui coule d'ouest en est et se jette dans la Cèze, près de la confluence de cette dernière avec le Rhône (figg. 1). Le gisement est situé à $500 \mathrm{~m}$ au nord du village, au pied d'une falaise du Turonien supérieur, ancienne ligne de rivage de la mer pliocène. Cette barre rocheuse abrite du mistral un versant en légère pente nord-sud, aménagé en terrasses et planté de vignes. Ce coteau est constitué par des dépôts d'origine éolienne dont la rapidité d'accumulation explique la bonne conservation des vestiges 


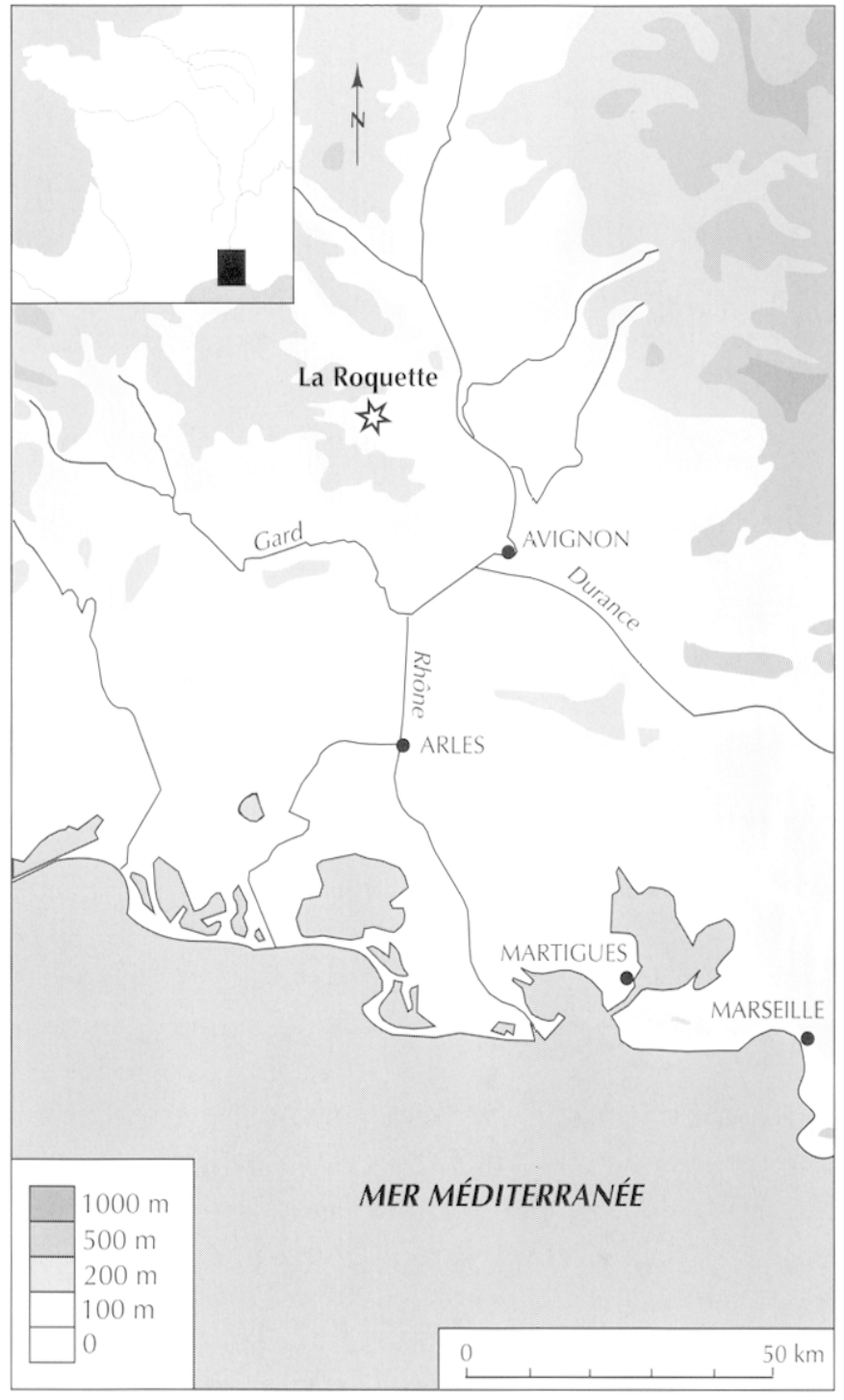

Fig. 1 - Localisation du site de La Roquette dans la basse vallée du Rhône (fonds de carte M. PY, CNRS, 1993).

(R. Arthuis, in verbis). L'opération archéologique est intervenue après des travaux d'arrachage de vignes dans deux parcelles voisines dénivelées de $1 \mathrm{~m}$ environ l'une par rapport à l'autre, et situées en amont de la pente à une cinquantaine de mètres de la falaise.

La vallée de la Tave s’intègre dans le pays des garrigues nord-gardoises, délimité à l'est par le Rhône, au nord par le canyon de la rivière Ardèche, à l'ouest par les premiers contreforts cévenols, au sud par le cours du Gardon et au sud-est par la plaine littorale du bas Rhône avec laquelle la basse vallée de la Tave est en contact.

\section{L'OCCUPATION DE LA VALLÉE DE LA TAVE AU NÉOLITHIQUE}

Au Néolithique final, la zone des garrigues nord-gardoises est densément occupée par le Ferrières. Elle se situe à cheval sur deux faciès de cette culture. Au sud, le faciès central occupe le pays de l'Uzège. Il est abondamment illustré dans les grottes du Gardon et présent dans la dépression d'Alès. Le faciès Cèze-Ardèche s'étend depuis la vallée de la Cèze et se poursuit au nord (Gutherz, 1984 ; Gutherz, Jallot, 1995). On peut approximativement délimiter une bande ouest-est de transition où sont mêlés les deux faciès. À l'est de cette zone, l'hypogée du Serre de Bernon à Laudun (Colomer, 1979; Gutherz, 1984) est pour l'instant le témoin le plus méridional du faciès Cèze-Ardèche, tandis qu'à l'ouest, ce stvle a été récemment retrouvé nettement au sud d'Alès, jusqu’à Boucoiran-et-Nozières (station de La Rouveyrasse haute, if. Bordreuil, 1998) et Saint-Maurice-deCazevieille (station du Montvièle, $c f$. id. ibid.). La vallée de la Tave se situe donc en limite des deux faciès. Elle fait l'objet de prospections depuis plusieurs années ; un réexamen des collections nous a permis de préciser l'ampleur de l'occupation de la vallée au Néolithique, et plus précisément au Néolithique final-Chalcolithique (fig. 2). La plupart des sites ainsi découverts sont inédits. Leur localisation est consignée dans les divers rapports de prospection (Petitot, 1992, 1993, 1994) et dans un mémoire de DEA (Petitot, 1991).

Le coteau de La Roquette, connu de longue date pour sa richesse archéologique, illustre la densité des occupations de la vallée depuis la Préhistoire. Des ramassages de surface y sont effectués depuis une vingtaine d'années ; ils montrent une fréquentation depuis le Paléolithique moyen jusqu'à l'époque romaine. Le Cardial lato sensu y est attesté par des tessons décorés de cordons verticaux dépassant du bord et par des anses en boudin. Du mobilier chasséen a été récolté en plusieurs points du lieu-dit. Dans une parcelle à environ $200 \mathrm{~m}$ du secteur fouillé, des fragments de céramique de diverses époques ont été recueillis; l'un d'eux, au moins, rappelle parfaitement certaines formes du Néolithique final découvertes lors de la fouille ; il s'agit d'un morceau de jarre profonde à lèrre impressionnée et languette de préhension. Dans une autre parcelle, située à environ $100 \mathrm{~m}$ de celles concernées par les excavations, ont été récoltés un grand fragment de coupe décoré de cannelures profondes en por- 


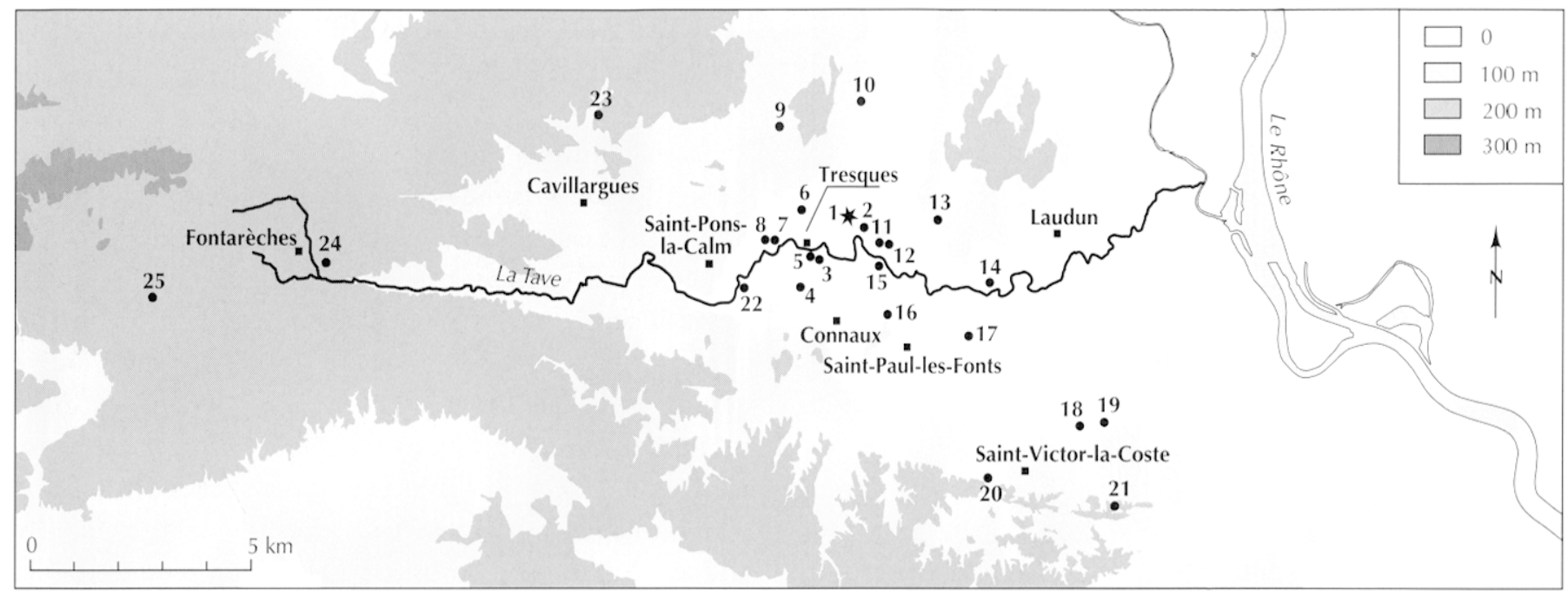

Fig. 2 - Emplacement des siles néolithiques de la vallée de la Tave (fonds de carte H. Petitot, 1997).

1-10, Tresques: 1, La Roquette; 2, Penchant sud de Bernon ; 3, Grange Neuve; 4, I.es Sablons; 5, La Berarde; 6, Saint-Martin ; 7, station de Pujols; 8, hypogée de Pujols ; 9, Roujaud ; 10, hypogée du Devois de l'Étang.

11-14, Laudun : 11, Serre de Bernon ; 12, Les Quatre Chemins ; 13, Plousole ; 14, Cranges de Nogier:

15, 16, Connaux : 15, Beaurert; 16, Fontaine du Crapaud.

17, Saint-Paul-les-Fonts, Cladier:

18-21, Saint-Victor-la-Coste : 18, Palus; 19, Marran; 20, grotte Canabif ; 21, aten Clauzel.

22, Saint-Pons-la-Calm, Dominargur.

23, Sabran, grotte de Brugiérolles.

24 , Fontariches, La Roque Viniere.

25, La Bruguiere, auen du Bois d'in Bas.

tée horizontale, un tesson orné d'une guirlande sur trois lignes traitées avec la même technique, une prise pseudofuniculaire, deux tessons à cordon impressionné et une préhension nasiforme. En l'absence de carène et de tout autre indice d'allure fontbuxienne, nous rattachons ces éléments au Ferrières. Enfin, à l'endroit où a été réalisée l'opération archéologique, un tesson caréné à bouton prismatique perforé situé sur la rupure de pente a été ramassé en surface. Il est attribuable au Fontbouisse.

Ailleurs, sur la commune de Tresques, les occupations antérieures au Néolithique final sont rares pour l'instant; seule une préhension multiforée chasséenne a été recueillie à Saint-Martin (A. Raux, in verbis). Sur la station du Penchant sud de Bernon, un vase sphéroïdal à décor de pastilles en portées longues horizontales se rapporte au Ferrières. La présence de cette culture est possible à la station de Pujols qui a livré un abondant matériel. Le Fontbouisse est présent sur de nombreux sites de la commune, en dehors de La Roquette : Les Sablons, La Berarde, Grange Neuve, Roujaud, I'hypogée et la station de Pujols (Raymond, 1900). L'hypogée du Devois de
l'Étang (Colomer, 1979) montre une production céramique particulière, presque inornée, dont les formes rappellent le Fontbouisse.

Dans les communes alentours, le Cardial lato sensu n'est représenté que par un tesson décoré d'impressions à la coquille découvert à Mayran, Saint-Victor-la-Coste (inédit) et par quelques tessons à cordons verticaux récoltés à I a Fontaine du Crapaud, Connaux (inédit). Une sépulure double fouillée par Henri Duday à Dominargue, Saint-Pons-la-Calm (fouille inédite) est attribuable au Chasséen, de même que le mobilier de la station de Cladier à Saint-Paul-les-Fonts. La densité des sites du Néolithique final-Chalcolithique est sans doute aussi importante qu'à Tresques mais les prospections dans ces communes ont été beaucoup moins soutenues. Le site le plus connu est l'hypogée ferrérien du Serre de Bernon à Laudun qui a fait l'objet d'une fouille (Colomer, 1979; Guther\%, 1984). Voisine de celui-ci, la station des Quatre Chemins (Laudun) a livré un matériel ténu, attribuable au Ferrières. Les sites de Plousole et des Granges de Nogier, sur la même commune, se rapportent à la culture 
de Fontbouisse. Dernièrement mis au jour, le site de Beauvert à Connaux a livré les vestiges d'un habitat fontbuxien (inédit). À Saint-Iictor-la-Coste, deux grottes sépulcrales fouillées anciennement (Gilles, 1962 ; Petitot, à paraitre), l'aven Clauzel et la grotte Canabié, ainsi que la station de Palus récemment découverte ont été occupées durant le Néolithique final et le Chalcolithique. La station de Cladier à Saint-Paul-les-Fonts a livré quelques tessons qui semblent se rapporter au Néolithique final ou au Chalcolithique. À Sabran, la grotte de Brugiérolles, récemment répertoriée (Petitot, à paraître), abrite probablement une sépulture collective. Un vase de forme simple à décor incisé de chevrons irréguliers en provient. Des prospections menées sur la commune roisine de Cavillargues ont livré un tesson présentant le même type de décor (H. Petitot, in verbis). À Fontarèches, une sépulture en coffre attribuable au Néolithique final ou au Chalcolithique a été fouillée par Jacques Vaton au lieu-dit La Roque Vinière (inédit). Pour finir, aux confins de la vallée de la Tave, dans l'aven du Bois d'En Bas (La Bruguière), a été récolté un petit lot de céramique non décorée (Bourgeois, Bourgeois, 1989; céramique inédite) très comparable à certains vases du Néolithique final de La Roquette.

Ce rapide aperçu de la vallée de la Tave permet de constater que la région est bien intégrée dans lc Languedoc oriental en matière d'occupation néolithique. Comme ailleurs dans le Midi, la présence humaine semble s'intensifier de manière significative à la fin de la période.

\section{ENVIRONNEMENT GÉOMORPHOLOGIQUE}

De récents travaux ont mis en évidence les traces d'un milieu marécageux holocène dans la basse vallée de la Tave (Ambert, 1994 ; Drapier, 1995). Sa misc cn place date du Boréal et résulte d'un engorgement de la rivière, depuis sa confluence avec la Cèze jusqu'en amont de Tresques, et sur 2 à $4 \mathrm{~km}$ de large. Les fluctuations qui ont sans doute affecté, au cours de son existence, le niveau et l'extension de la zone palustre ne sont pas connues. L'accélération de l'anthropisation, que l'on peut situer entre la fin du Néolithique et l'époque romaine, semble jouer un rôle décisif dans la résorption du marécage. La présence de l'homme contribue en effet à enrayer le processus de formation des travertins, en partie responsable de l'engorgement du réseau (P. Ambert et R. Arthuis, in verbis). Il faut donc peut-ĉtre envisager, pour le site de $\mathrm{La}$ Roquette, un environnement humide, végétal et faunistique différent de l'actuel, sanıs qu'il soit possible de préciser ses caractéristiques et les répercussions éventuelles sur le mode de vie des habitants.

Bien que l'on ne soit pas encore en mesure d'attester l'existence de ce milieu durant le Néolithique, on observe que La Roquette, tout comme la grande majorité des gisements des périodes pré- et protohistoriques de ce secteur, est située sur un niveau de terrasse surplombant le lit majeur de la Tave d'une vingtaine de mètres.

\section{DÉROULEMENT ET CONDITIONS DE LA FOUILLE}

Dans une zone de sédiment riche en matière organique, sept sondages de 2 et $4 \mathrm{~m}^{2}$ chacun ont d'abord été ouverts; six d'entre eux se sont révélés positifs. Dans un deuxième temps, une surface de $95 \mathrm{~m}^{2}$, englobant la plupart des sondages, a été décapée. Enfin, une tranchée ouverte au sud de la surface fouillée s'est elle-même révélée positive (fig. 3).

La fouille a été réalisée en quatre mois. Les observations planimétriques n'ont pas été consignées. Le niveau archéologique ainsi que les fosses ont été fouillés "d'un bloc ", sans l'aide de décapages artificiels. L'enregistrement des données a été sommaire. Dans les niveaux archéologiques, seuls des amas de tessons ont été localisés en plan. Le matériel n'a pas été coté en profondeur. Les relevés ne concernent que les aménagements en creux (plans et sections).

\section{INDICATIONS STRATIGRAPHIQUES}

La stratigraphie est la suivante (fig. 4) :

- couche 1 : labours ; cette couche remaniée est d'épaisseur constante $(0,15 \mathrm{~m}$ environ) ;

- couche 2a : limon sableux brun foncé, organique ; elle est en contact avec la terre arable; elle est remaniée dans sa partie supérieure comme le montrent deux structures de combustion démantelées. Son épaisseur varie de 0,5 à $0,25 \mathrm{~m}$; elle renferme de nombreux vestiges;

- couche $2 b$ : cailloutis jaune induré; elle est épaisse d'une dizaine de centimètres et s'amenuise au sud de la 


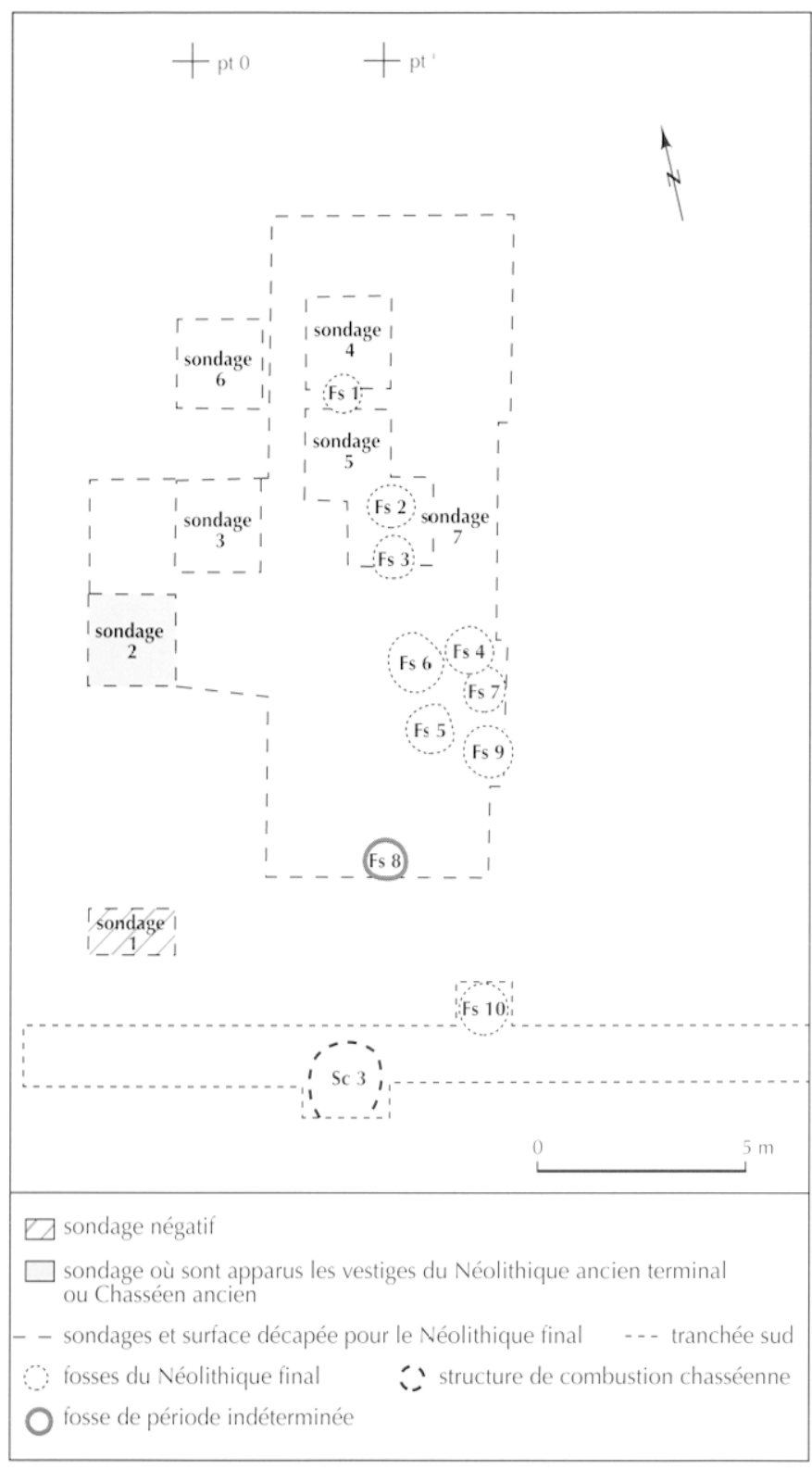

Fig. 3 - Plan général de la zone fouillée aver la disposition des principales structures: Is, fosse; $S$, structure de combustion.

surface décapée jusqu'à disparaitre au niveau de la tranchée sud ; elle est formée d'un apport de graviers anguleux et de sable grossier provenant des abords du site; elle est soigneusement égalisée et épouse la déclivité naturelle des couches sous-jacentes; son origine anthropique et sa fonction de sol aménagé ne font pas de doute ;

- couche 3 : limon sableux brun clair, stérile; elle est épaisse de $0,30 \mathrm{~m}$;

- couche 4 : sable argileux brun-jaune (substrat).

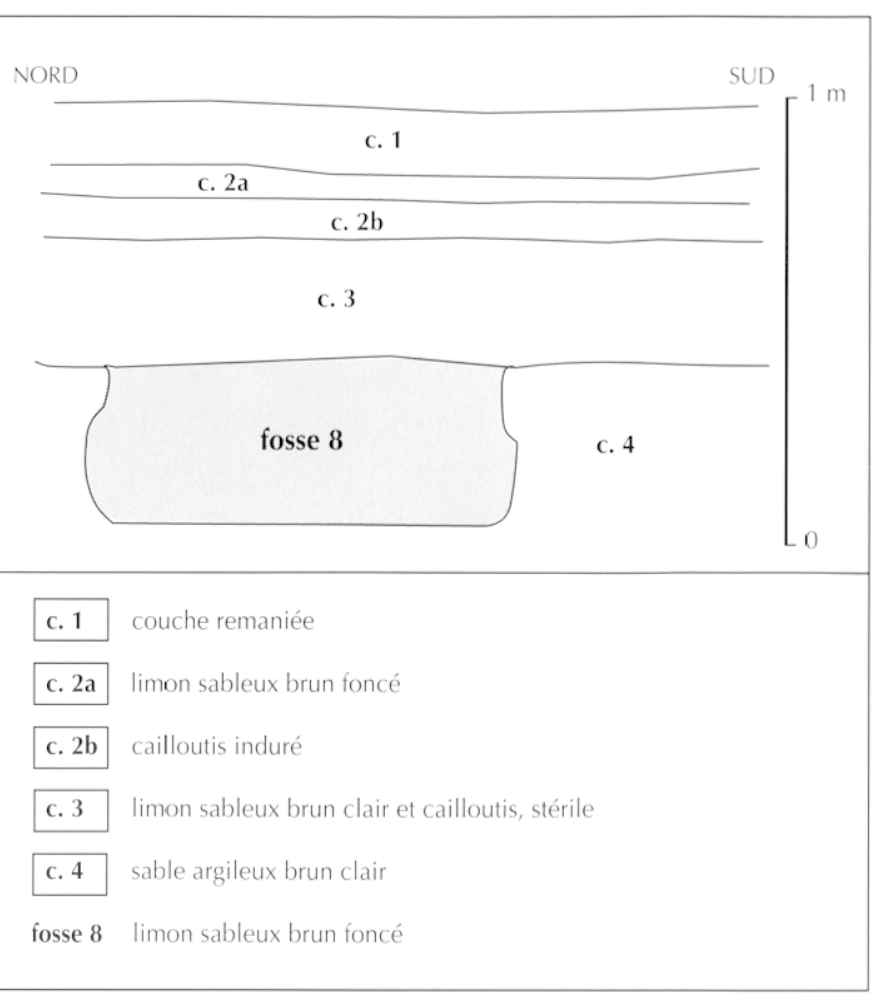

Fig. 4 - Straligraphie observée au nivean de la fosse 8 de La Roquette.

\section{LES OCCUPATIONS ANTÉRIEURES AU NÉOLITHIQUE FINAL}

\section{LE NÉOLITHIQUE ANCIEN TERMINAL OU CHASSÉEN ANCIEN}

Une nappe de sédiment riche en matière organique de $2 \mathrm{~m}^{2}$, repérée dans le sondage 2 , a livré des tessons, quelques ossements animaux et une lamelle de silex beige. Le lien stratigraphique de ces vestiges avec le reste du gisement n'a pas pu être établi.

Deux formes céramiques sont reconstituables (fig. 5). La première est une bouteille à col rétréci et éversé possédant trois anses en ruban; la seconde est une petite coupe hémisphérique à anse en ruban. Les autres tessons sont peu caractérisés, à l'exception de plusieurs anses en ruban qui sont les seuls éléments de préhension.

La disposition de la bouteille semble intentionnelle. Elle reposait à l'envers, sur son col, solidement calée par des pierres dont deux broyons de grès. Le fond du vase avait été découpé (traces visibles) et déposé dans le gou- 
lot sur sa convexité. L'ensemble s’assimile manifestement à un dépôt d'objets.

Les pâtes de ces tessons paraissent fragilisées par la présence d'un grand nombre de grains de dégraissant calcaire grossier (entre 2 et $7 \mathrm{~mm}$ ), parfois brûlés, mal répartis dans la pâte. La couleur des surfaces est plus ou moins homogène; elle varie du beige foncé au gris. Les parois sont lissées.

Les formes reconstituées sont ubiquistes dans le Néolithique, mais la présence d'anses en ruban, à l'exclusion de tout autre élément de préhension, évoque le Néolithique ancien et le Chasséen ancien où les bouteilles à col rétréci sont bien représentées. I a disposition ternaire des anses évoque davantage le Néolithique ancien cardial ou épicardial que le Chassécn où elle est rarissime.
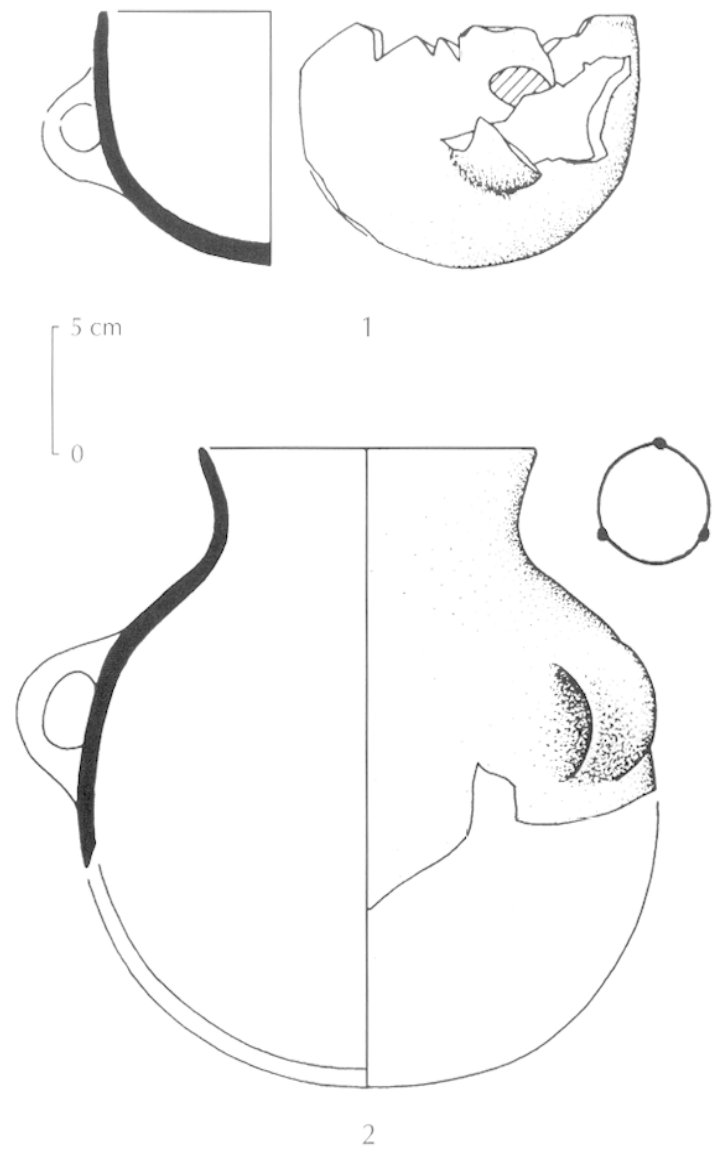

Fig. 5 - Céramique du sondagr 2 (dépôt du . Nöolithique ancien final ou du (hassépn ancien): 1, bouteille à trois anses en mban; 2, coupe hémisphérique à anse en ruban.

\section{LE CHASSÉEN}

Dans la tranchée ouverte au sud de la fouille est apparue une strucure de combustion (Sc 3) à laquelle était associé un abondant matériel. Ayant été découverte durant les derniers jours de la fouille, cette structure n'a fait l'objet d'aucun relevé. Le lien stratigraphique de ces restiges avec le reste du gisement n'a pas pu être établi.

Cet aménagement se situe dans une couche de sable grossier et repose sur une lentille argileuse fine. Il est constitué de plaques de torchis formant un amas de $1 \mathrm{~m}$ de diamètre sur $0,40 \mathrm{~m}$ d'épaisseur. Ces plaques étaient à l'origine assemblées comme des colombins et formaient une structure en élévation dont une petite partic était en place lors de la fouille. Le diamètre à la base était de $0,60 \mathrm{~m}$. La rubéfaction du sédiment au fond de la structure et des plaques elles-mêmes, ainsi que l'abondance des fragments de céramique, dont certains ont recuit au pied de la structure, ont conduit le fouilleur à formuler l'hypothèse d'un four. Bien qu'à notre connaissance aucun four en élération n'ait jamais été signalé avec assurance dans le Néolithique méridional, d'autres sites montrent des indices dans cette voie. C'est le cas de Roquemengarde (Saint-Pons-de-Mauchiens, Hérault) où, parmi les aménagements soigneusement étudiés, figurait " un anlas de plaques de torchis aux formes souvent courbes " en association probable avec un muret de pierres que les fouilleurs avouent ne pouroir "guère interpréter que comme un four " (Guilaine, Coularou, 1986, p. 4).

La céramique se compose de formes typiques du Chasséen (fig. 6) : une écuelle à carène vive et paroi courte très éversée, le fond d'une coupe à décor arciforme s'organisant autour d'un bouton perforé, un grand rase à col, un rase à épaulement portant un bandeau multiforé, un gobelet tulipiforme à lèvre crénelée, un pot ovoïde, une bouteille globuleuse à col court et un vase sphéroïdal à ouverture étroite possédant tous deux des boutons à perforation sous-cutanée.

L'industrie lithique se compose presque exclusivement de lamelles fines et régulières en silex «blond " à grain fin, translucide (fig. 6). Ces lamelles sont très peu retouchées. On note également la présence d'une fusaiole en pierre.

L'industrie osseuse est représentée par la partie distale brûlée d'une pointe fine sur os scié longitudinalement et poli. 

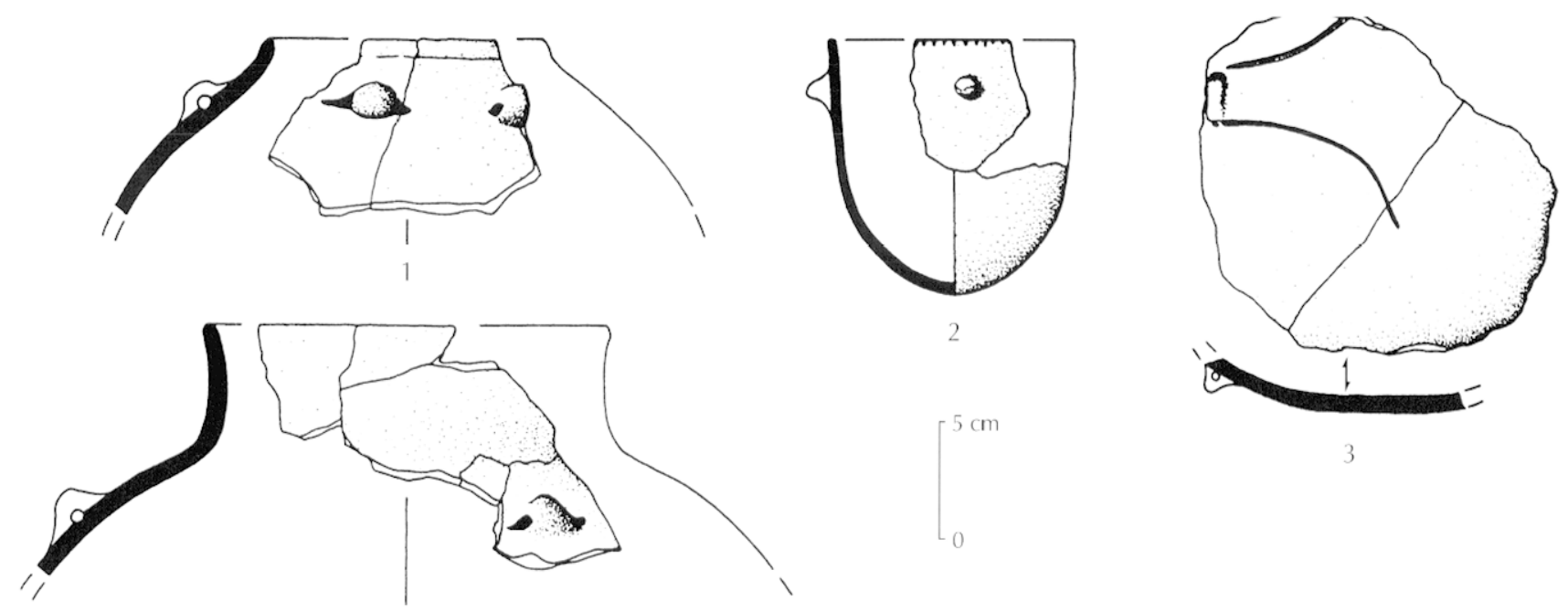

4

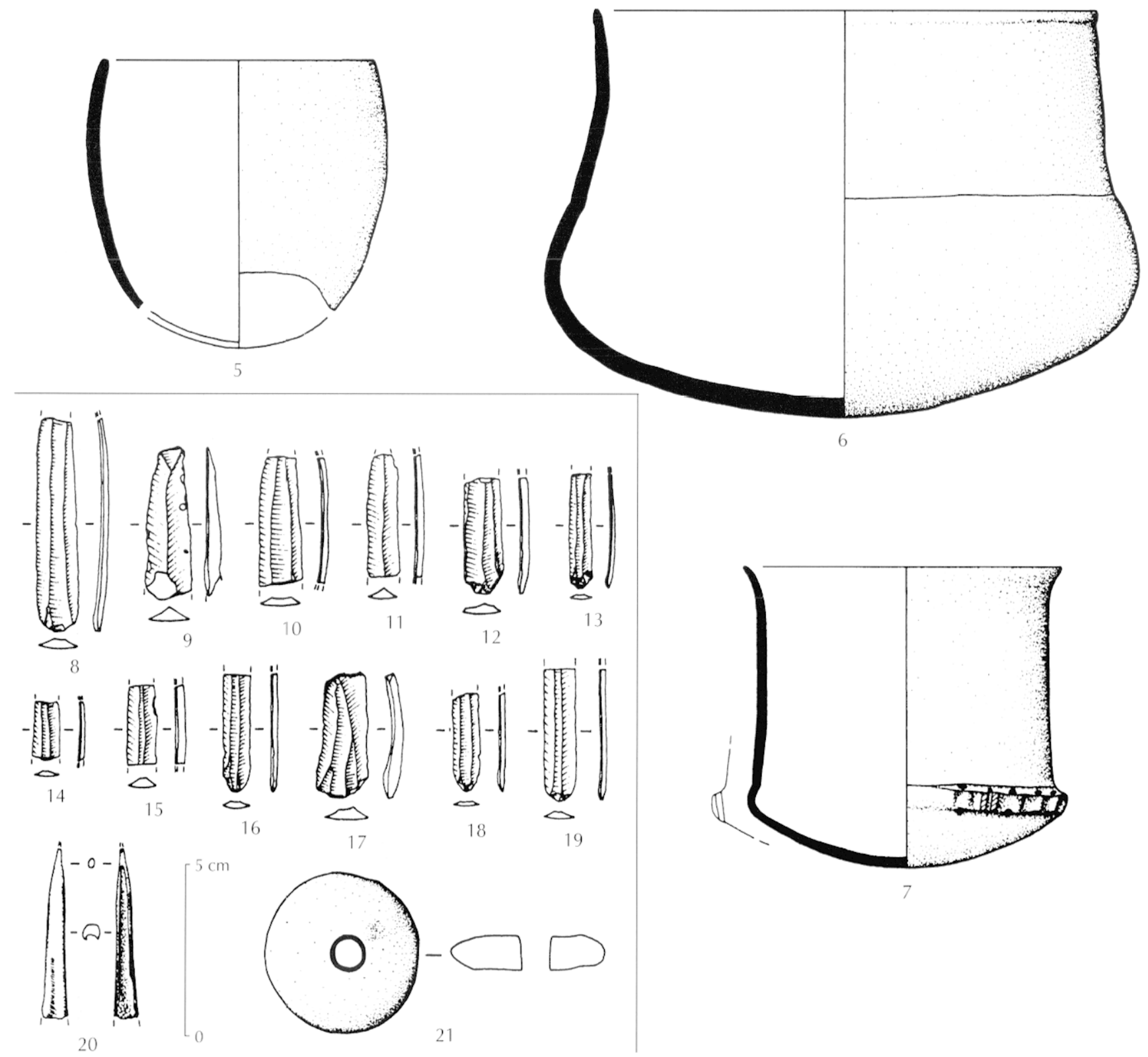

Fig. 6 - Mobilier chasséen de la structure de combustion Sc 3:1, vase globuleux à ouverture étroite; 2, vase tulipiforme à lève crénelée ; 3, fragment de coupe à décor en moustache; 4, vase globuleux à col court ; 5, vase ovö̈de; 6, grand vase à épaulement ; 7, vase à épaulement et cordon multiforé; 8-19, lamelles non retouchées en silex "blond";20, pointe sur os scié d Ovis-Capra ; 21, fusaïole en pierre. 
Le matériel chasséen associé à cette structure se classe sans doute dans une phase récente selon la chronotypologie élaborée par Alain Beeching dans la zone Cèze-Ardèche (Beeching, 1995). Plusieurs éléments sont à rapprocher du groupe $C$ : le décor en moustache, le vase à épaulement et bandeau multiforé, la coupe à carène vive, ainsi que du groupe $\mathrm{E}$ : le vase cylindrique à bandeau multiforé et le vase ovoïde. Les datations de ces deux groupes convergent vers la fin du $V^{c}$ millénaire et le début du $I^{c}$ avant notre ère. Cet ensemble est également proche du style d'Auriac, identifié par Jean Vaquer (1990, 1991) dans le bassin de l'Aude et attribuable au premier quart du $\mathrm{IV}^{\mathrm{rc}}$ millénaire avant J.-C.

\section{LE NÉOLITHIQUE INDÉTERMINÉ}

Une fosse (Fs 8), située dans l'aire décapée et s'ouvrant au sommet de la couche 4 , a été rattachée à l'occupation chasséenne par le fouilleur (fig. 4). Son ouverture est rétrécie, elle est peu profonde $(0,35 \mathrm{~m})$ et son fond est plat. Elle a livré trois lamelles en silex blond et quelques menus tessons, dont plusieurs profils sinueux et un col fermé (fig. 7). Aucune carène n'est présente. Un grand fragment de jarre montre, sur le haut de la panse, une rangée d'impressions oralaires fortement imprimées dans la pâte. Ce tesson ne s'apparente guère au Chasséen, et aucun des décors imprimés du Néolithique final sus-jacent ne l'est aussi profondément. D'autre part, une perturbation de la fosse par la couche du Néolithique final sus-jacent paraît difficilement envisageable dans la mesure où elle en est séparée par une couche stérile de $0,30 \mathrm{~m}$ d'épaisseur. Les décors imprimés étant présents au Néolithique ancien, une corrélation avec le matériel du sondage 2 peut être envisagée, mais les pâtes sont ici très différentes de celles, bien typées, décrites précédemment pour la bouteille à trois anses et la coupe hémisphérique ( $c f$. supra, p. 259). Enfin, il serait tentant d'imaginer que cette fosse s'insère chronologiquement entre l'occupation chasséenne et celle du Néolithique final, mais ce que l'indigence du matériel recueilli ne peut nous assurer, les indications stratigraphiques ne permettent pas darantage de le démontrer. L'attribution chronoculturelle de cette fosse reste donc problématique.
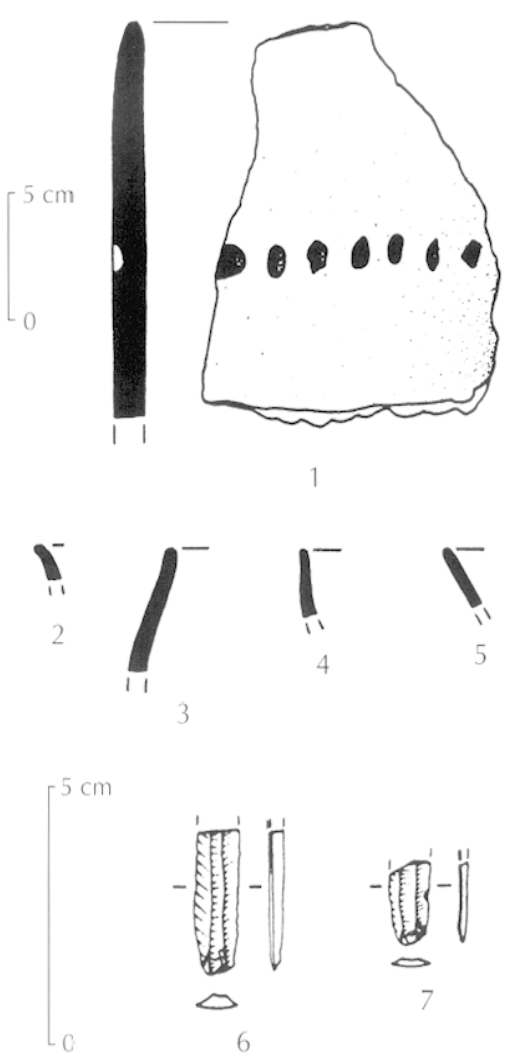

Fig. 7 - Mobilier de la fosse 8 (Néolithique indéterminé) 1, fragment de jarre décoré d'impressions ovalaires ; 2-5, bords divers ; 6, 7, lamelles en silex blond.

\section{L'OCCUPATION DU NÉOLITHIQUE FINAL}

Cette occupation a été mise au jour sur une surface de $93 \mathrm{~m}^{2}$ environ.

\section{LES AMÉNAGEMENTS}

Huit fosses s'ourrent à la surface du sol induré (c. 2b), ainsi que d'autres creusements peu profonds, circulaires ou semi-circulaires en plan. Deux trous de poteau, cerclés par de gros blocs de calage, s'ouvrent également à ce niveau. Plusieurs amas de pierres parfois brûlées apparaissent dans la couche $2 \mathrm{a}$, mais leur organisation n'a pas pu être perçue. Au sommet de la couche 2a ont été obserrées deux structures de combustion démantelées par les travaux agricoles dont il ne restait que les taches circulaires de charbon (fig. 8). Dans l'une des deux a été découvert un fragment de mâchoire de chien. Enfin, la 


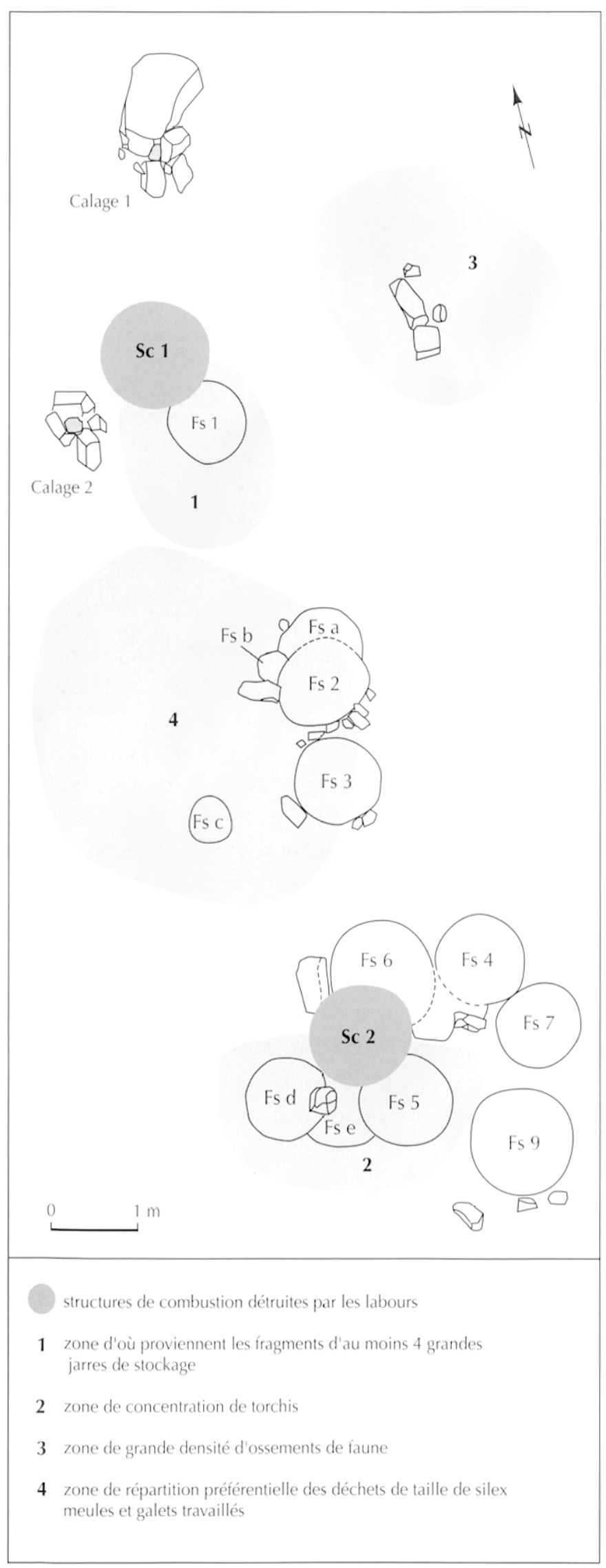

tranchée effectuée au sud de la fouille a montré l'existence d'une fosse de cette période mais le sol induré est absent à cet endroit et cette structure est largement tronquée par l'érosion.

Les fosses sont approximativement réparties selon un axe nord-sud dans le périmètre décapé (fig. 9). Certaines d'entre elles sont très proches les unes des autres et le sol induré n'existe pas entre elles (Fs 5, 6 et 7). Deux fosses également voisines sont séparées par un muret de trois rangs de pierres hétérogènes (Fs 2 et Fs 3). Les dimensions de ces fosses varient de 1,10 à $1,50 \mathrm{~m}$ de diamètre à l'ouverture et de 0,40 à $1,20 \mathrm{~m}$ de profondeur. Leur ouverture est soit rétrécie, déterminant une forme "en sac" (Fs 1, 4 et 5), soit évasée (Fs 3, 6, 7), soit droite (Fs 9). Leur fond est presque toujours aplati. Leur comblement est généralement homogène; il est constitué d'un limon sableux brun foncé organique de même nature que celui de la couche $2 a$. Le comblement de la fosse 5 , de même que celui de deux petites dépressions situées aux alentours de cette fosse, est presque exclusivement constitué de torchis. La fosse 7 , la moins profonde et la plus large, possède un comblement très cendreux. Elle contenait de nombreuses esquilles d'os brûlées. Enfin, deux fosses (Fs 1 et Fs 4) sont scellées par des blocs hétérométriques au niveau de leur ouverture.

Outre la présence de céramique et d'industrie lithique et osseuse dans le remplissage des fosses, on note celle d'un squelette de chien en connexion dans la fosse 9 .

Les creusements peu profonds (fig. 8, Fs a à e) ont des profils divers. Leur profondeur est comprise entre 20 et $35 \mathrm{~cm}$. Leur paroi est verticale, leur fond est plat ou en cuvette ( $F s$ d). Leur comblement n'est pas de même nature que celui des grandes fosses par lesquelles ils sont recoupés. Il s'agit d'un sédiment non organique contenant un très rare mobilier à l'exception du torchis. Fs a, qui se développe en demi-lune autour de la fosse 2, ne peut, sclon le fouilleur, être assimilée à un effondrement de paroi de cette dernière. Le comblement de Fs a est constitué par un cailloutis qui pourrait avoir servi à étayer l'ouverture de la fosse 2 , de la même manière que le "muret " situé entre Fs 2 et Fs 3 a pu renforcer la paroi

Fig. 8 - Emplacement des diverses structures du Néolithique final et zomes de répartition préférentielle de certains types de vestiges : Fs, fosse; $\mathrm{S}$, strurture de combustion. 


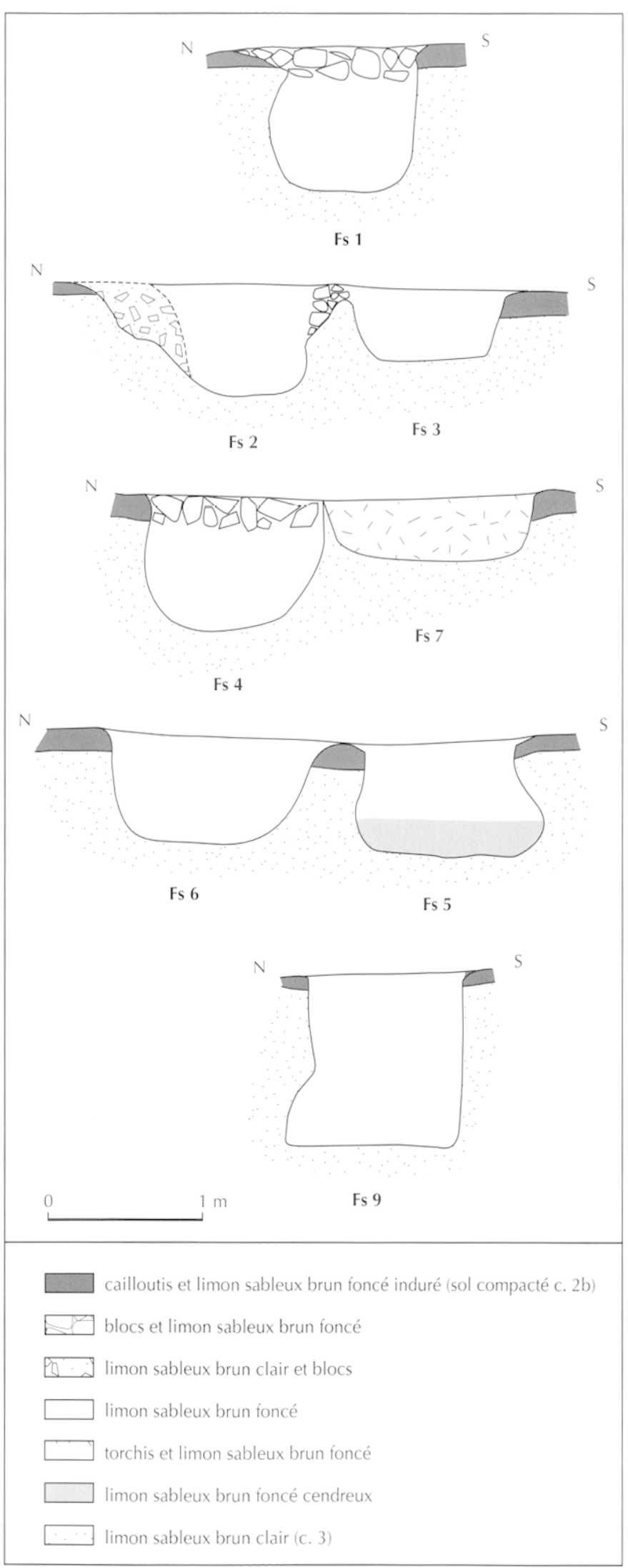

Fig. 9 - Coupes des fosses du Néolithique final de La Roquette. mitovenne de ces deux structures. La fonction des autres creusements est difficile à déterminer.

Le mobilier est abondant dans toute la couche archéologique et dans les strucures. La céramique connporte 3234 tessons. L'industrie lithique est beaucoup plus restreinte : 235 pièces débitées ont été dénombrées, en majorité des éclats dont une quarantaine seulement portent des retouches. Les lamelles sont plus nombreuses que les lames. L'outillage poli compte cinq lames entières ou fragmentées. L'industrie osseuse est représentée par 25 pièces, en majorité des outils perforants. Cinq éléments de parure sont présents. On note également de nombreux fragments épars de torchis dont certains portent des empreintes de brindilles.

\section{RÉPARTITION DU MOBILIER ET ÉVOLUTION FONCTIONNELLE DU SITE}

La concentration de certains types de vestiges montre une répartition des activités domestiques sur la surface décapée (fig. 8).

Un ensemble important d'ossements animaux est situé au nord-est de la fouille ( $c$. infra, annexe, p. 287291). La majorité des récipients de stockage (quatre au moins) ont été décuuverts aux alentours de la fosse 1, au nord du décapage. Au centre, les fosses 2 et 3 sont environnées de déchets de taille de silex (éclats et blocs), alors que les produits du débitage lamellaire et la majorité des outils ont été retrouvés dans les fosses. C'est également aux alentours des fosses 2 et 3 que sont situés, dans leur majorité, les meules et les galets qui ont pu être utilisés. Enfin, dans la partie sud où sont groupées cinq fosses, les fragments de torchis sont nombreux, y compris dans certaines de ces structures. Aucune graine n'a été retrouvée à la fouille.

Quelle qu'ait été la fonction originelle des fosses principales (probablement des silos pour la plupart), elles ont toutes été réutilisées. L'abondance du mobilier recueilli dans ces structures et la nature du sédiment de remplissage, impossible à différencier de celui de la couche $2 \mathrm{a}$ d'après le fouilleur, plaident pour une fonction ultime de type dépotoir.

Le rapport entre les fosses principales et les creusements peu profonds est difficile à appréhender. I.es recoupements de ces structures entre elles semblent dus à des réaménagements dont on ne peut préciser la 
nature, excepté, peut-être, dans le cas de Fs a (cf. supra, p. 262).

Vers la fin de l'occupation, la fonction du site semble avoir totalement changé puisquc lcs aménagements se réduisent alors à deux foyers, très mal conservés. Les modifications qui s'opèrent peu à peu au niveau des activités pratiquées sur le site ne peuvent pas être davantage précisées, faute de fouille fine. On peut seulement suggérer l'existence d'une aire de rejet ou de travail de boucherie (concentration d'ossements), d'une aire de rangement des jarres de stockage et d'un atelier de taille de silex. On peut admettre également l'existence d'une structure en élévation sur ou aux abords de l'aire fouillée d'après l'abondance de torchis et la présence des calages. Tous ces éléments évoquent un habilat stricto sensu.

\section{LE MOBILIER}

\section{LES INDUSTRIES LITHIQUES}

\section{L'outillage taillé}

Les produits lamellaires sont représentés par une trentaine d'individus, en majorité des lamelles souvent très fragmentées (fig. 10). Ces pièces portent généralement des retouches continues ou discontinues sur un ou deux bords, plates ou semi-abruptes, marginales, le plus souvent directes. On dénombre, en outre, des grattoirs sur distal ou sur proximal ( $\mathrm{n}^{\text {os }} 3,6$ et 11$)$, un burin d'angle $\left(n^{\circ} 10\right)$, un fragment distal appointi par retouche abrupte $\left(n^{\circ} 12\right)$, une lamelle à bords abattus $\left(n^{\circ} 15\right)$ et un fragment de lame épaisse et étroite vraisemblablement foliacée et calcinée.

Les talons sont lisses ou facettés, rarement punctiformes. La percussion directe est attestée par un ou deux enlèvements perpendiculaires au talon, présents sur plusieurs fragments de proximal $\left(\mathrm{n}^{\prime \prime} 3\right.$ et 7$)$. Les nervures peuvent être très régulières sur de petits fragments de lamelles et sur certaines lames. Plusieurs exemplaires ont une partie corticale.

Une seule lamelle est entière; elle est courte et trapue (longueur : $3,9 \mathrm{~cm}$; largeur : $1,1 \mathrm{~cm}$ ).

Hormis deux ou trois fragments qui montrent une finesse et une régularité dignes des lamelles chasséennes - deux d'entre eux sont d'ailleurs réalisés dans le même matériau que les exemplaires provenant de la structure de combustion chasséenne (Sc 3) - l'épaisseur des lames et lamelles est supérieure à celle des pièces chasséennes. Leur rapport largeur/épaisseur donne 3,93; il est de 4,85 pour les lamelles chasséennes de la structure de combustion.

De nombreuses pièces ont subi une chauffe plus ou moins intense postérieurement à leur façonnage.

L'industrie sur éclat (fig. 10) compte 29 pièces retouchées dont un perçoir $\left(n^{\circ} 23\right)$, un grattoir à épaulement $\left(n^{\circ} 13\right)$, deux grattoirs carénés ( $n^{\circ *} 9$ et 14$)$, un grattoir cortical à proximal aménagé $\left(n^{\circ} 20\right)$, un grattoir simple cortical $\left(n^{\circ} 5\right)$, un racloir convexe $\left(n^{\circ} 21\right)$, une curieuse pièce allongée $\left(n^{\circ} 22\right)$, corticale, dont la partie active, fine, bien dégagée et très irrégulière montre une fine retouche continue (couteau à dos cortical ?), une pointe losangique $\left(\mathrm{n}^{\circ} 26\right)$ à retouche bifaciale envahissante et partie proximale légèrement dégagée par retouche abrupte. On trouve également de nombreuses pièces présentant des retouches minces localisées qui s'assimilent souvent plus à l'utilisation qu'à un réel façonnage. Les pièces possédant une partie corticale sont nombreuses, ce qui montre le faible indice de transformation des nucléus. Plusieurs autres portent des traces de chauffe postérieures au débitage.

Les éclats non retouchés sont prépondérants; nous en avons compté 125. Ils sont souvent épais et larges. Nombre d'entre eux ont, encore plus que les éclats retouchés, une partie corticale importante. Les stigmates de chauffe sont également souvent présents.

Les matériaux utilisés semblent avoir une origine locale, excepté peut-être le rare silex "blond "; il s'agit d'un silex miel ou brun moyen fréquent dans la région, notamment au nord d'Uzès (silex du Rouziganet; Gutherz, in verbis), ou foncé à cortex blanchâtre moucheté de quartzites et de matériaux gréseux divers que l'on trouve aux abords du site ou dans la rivière Tave.

Une soixantaine de blocs de formes diverses, souvent corticaux, mesurant entre 3 et $9 \mathrm{~cm}$ sont mêlés aux déchets de taille. Il est souvent très difficile d'identifier des négatifs d'enlèvement en raison de la mauvaise qualité des matériaux qui sont presque exclusivement de type gréseux. Certains de ces blocs portent des retouches localisées. Les supports de débitage identifiés comme tels sont peu nombreux : deux nucléus en quartzite et un en silex. Le premier se présente 
comme une petite pièce conique polyédrique; le deuxième, plus gros et grossièrement sphérique, montre des enlèvements en tous sens sur toute sa surface; le dernier, en silex, de petite taille (diamètre : $3,5 \mathrm{~cm}$ ), est tiré d'un petit galet dont il a gardé l'enveloppe corticale sur plus d'un tiers de sa surface. Les enlèvements ont là encore été effectués à partir de multiples plans de frappe.

La répartition de l'industrie lithique dans la fouille fait ressortir une aire de débitage au centre de la surface décapée, dans laquelle se trouvent essentiellement les blocs, les nucléus et les gros éclats. Les lames et lamelles, les outils sur éclat ainsi que les esquilles (moins de $2 \mathrm{~cm}$ ) ont été récoltés dans les fosses. Le débitage concerne essentiellement le quartzite et le grès. L'exploitation du silex, dont témoignent les lames et lamelles et quelques outils sur éclat, ne s'effectuait sans doute pas sur place, du moins pas dans l'aire fouillée.

\section{L'outillage poli}

L'outillage poli est représenté par deux haches entières de petite taille (moins de $4 \mathrm{~cm}$ ). L'une des deux, de forme quadrangulaire allongée, possède un tranchant très acéré ; elle est polie sur toute la surface (fig. 10, $\mathrm{n}^{\circ} 28$ ). L'autre est plus large et plus plate; son polissage est moins intense et laisse apparaitre le bouchardagc (fig. 10, $n^{\circ}$ 29). Le tranchant porte, sur une face, de petites stries perpendiculaires d'usure. Un fragment distal semble provenir d'une hache de même module. Ces trois exemplaires sont en roche verte.

Un autre distal, appartenant à un outil de module nettement supérieur, possède un tranchant légèrement émoussé. De longues stries obliques et régulières, probablement dues au façonnage, affectent ses deux faces. Il est également en roche verte.

Un outil poli de grandes dimensions présente de multiples fractures dans le sens de la longucur qui ont sectionné l'objet en deux et dégagé des esquilles plates. Cette fragilité est peut-être due à la nature du matériau (roche grise schisteuse). La partie distale et le talon ont été tronqués. Les parties intactes montrent un polissage uniforme. La longueur conservée de cet outil est de $7 \mathrm{~cm}$.

\section{Le matériel de broyage}

Il est représenté par huit fragments de meules ou broyeurs en grès rose d'environ $0,20 \mathrm{~m}$ de long, 0,14 à
$0,18 \mathrm{~m}$ de large et 0,6 à $0,8 \mathrm{~m}$ d'épaisseur. La face active est plane ou légèrement bombée.

\section{L'OUTILLAGE EN MATIÈRE DURE ANIMALE}

L'outillage osseux est représenté par 23 pièces très bien conservées, qui se prêtent à une observation visuelle des traces de façonnage (fig. 11). La détermination des espèces a été faite par Evelyne Crégut et Vianney Forest.

\section{Les pointes}

Les pointes sont les plus nombreuses (18). Neuf pièces sont façonnées sur os longs de petits ruminants parmi lcsquels les caprinés concernent au moins sept outils. Un exemplaire, dont il ne reste que la partie proximale, est pris sur un tibia de lapin et un autre sur un humérus de chien. Une pointe est tirée d'une incisive de porc ou de capriné. Enfin pour six pointes dont il ne reste que la partie active, leur identification n'est pas possible.

Trois pointes ont été obtenues à partir d'os fendus (fig. 11) : une pointe à épiphyse en poulie sur un métapode de capriné $\left(\mathrm{n}^{\circ} 2\right)$, une pointe sur un tibia de capriné $\left(n^{\circ} 1\right)$ et une pointe provenant probablement du même type d'os que la précédente $\left(n^{\circ} 8\right)$. Les méthodes de fabrication de ces objets sont très difficiles à distinguer à l'œil nu, car elles sont totalement masquées par les stigmates de façonnage. Ces derniers se présentent sous la forme de stries obliques visibles sur toutes les faces ou sur la face inférieure seulement. Ces stries résultent d'une abrasion réalisée soit au polissoir dur, soit sur meule dormante.

Une pièce technique sur un métapode de capriné $\left(n^{\circ} 13\right)$ a conservé, sur sa face inférieure, les longues rainures très nettes du sciage longitudinal. Celles-ci ne parviennent pas tout à fait jusqu'au c'an'al médullaire, en bordure duquel un léger ressaut visible de chaque côté atteste l'utilisation de la percussion pour détacher les deux parties de l'os. Des stries de mise en forme, présentes sur toute la face supérieure de l'objet, s'arrêtent net là où commencent les rainures du sciage, montrant vraisemblablement l'antériorité d'une étape du façonnage par rapport au débitage.

Quatre pointes sont façonnées sur des os entiers : l'une est tirée d'un tibia de capriné (mouton, $\mathrm{n}^{\circ} 3$ ) ; une autre, dont il ne reste que la partie proximale, est prise 

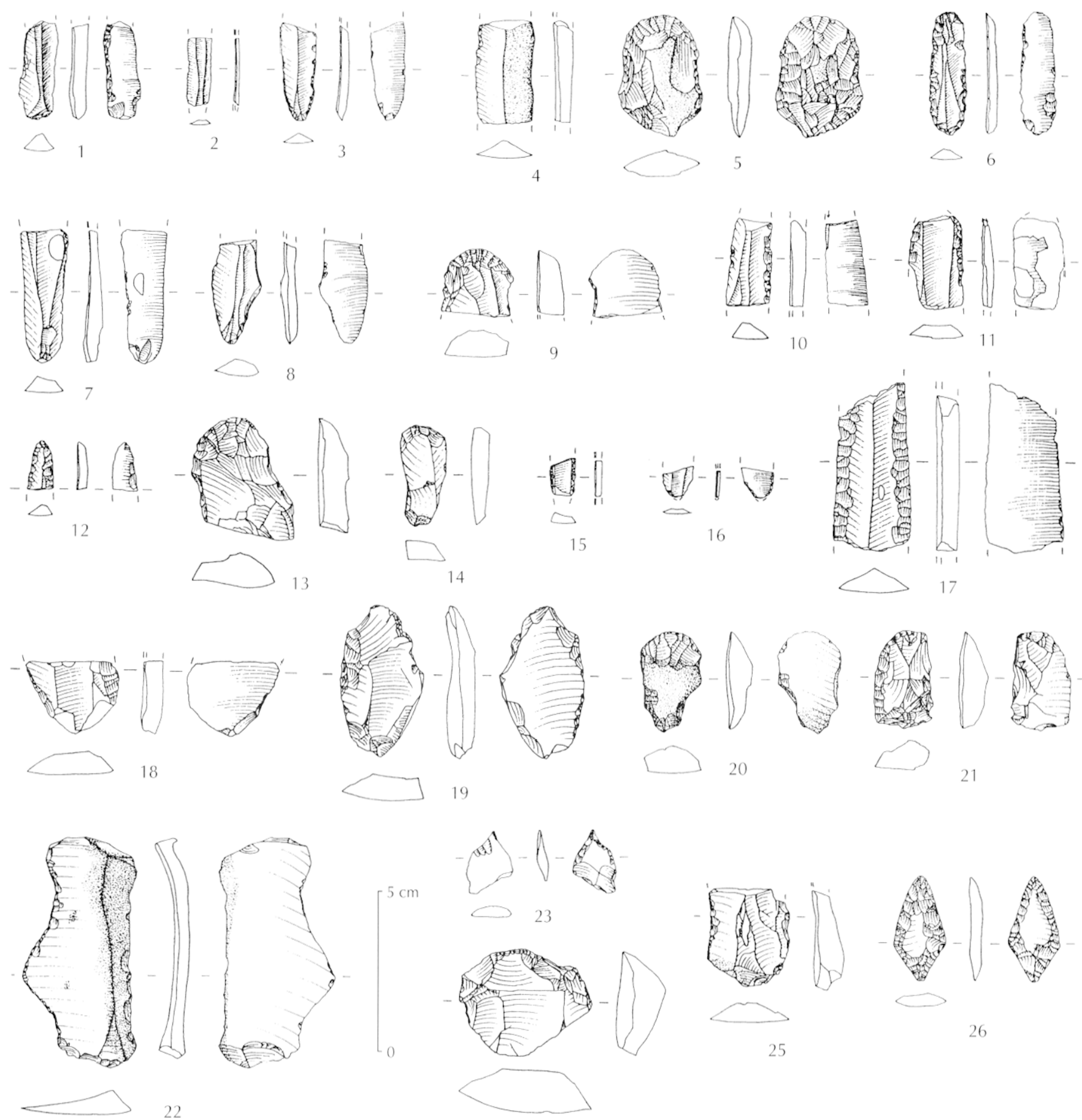

24
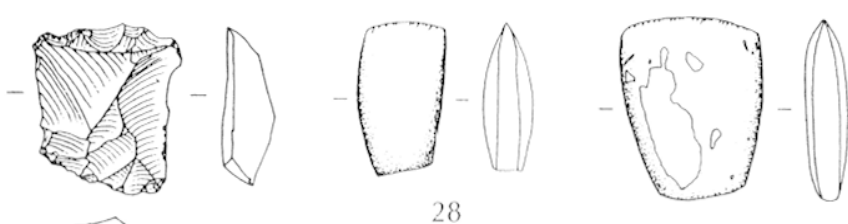

Fig. 10 - Industrie lithique du Néolithique final (fosses, couches 2 at et 2b) : 1, 10, 15, 17, 18, 25, lames et lamelles retouchés; 2, 4, 7, 8, 16, lames el lamelles non retouchées ou à retouche fine (d'usure?) ; 3, 6, 11, grattoirs sur produits lamellaires; 5, 9, 13, 14, 20, graltoirs sur éclat ; 10, burin d'angle; 12, fragment distal appointi par retouche abrupte; 15, lamelle à bords abattus; 19 , éclat à retouche marginale bifaciale; 21 , racloir convexe; 22 , pièce allongée à fine retouche contimue; 23 , pergoir ; 24, 27, éclats divers retouchés; 26 , pointe losangique à retouche envahissante; 28,29 , lames de hache en roche verte. 

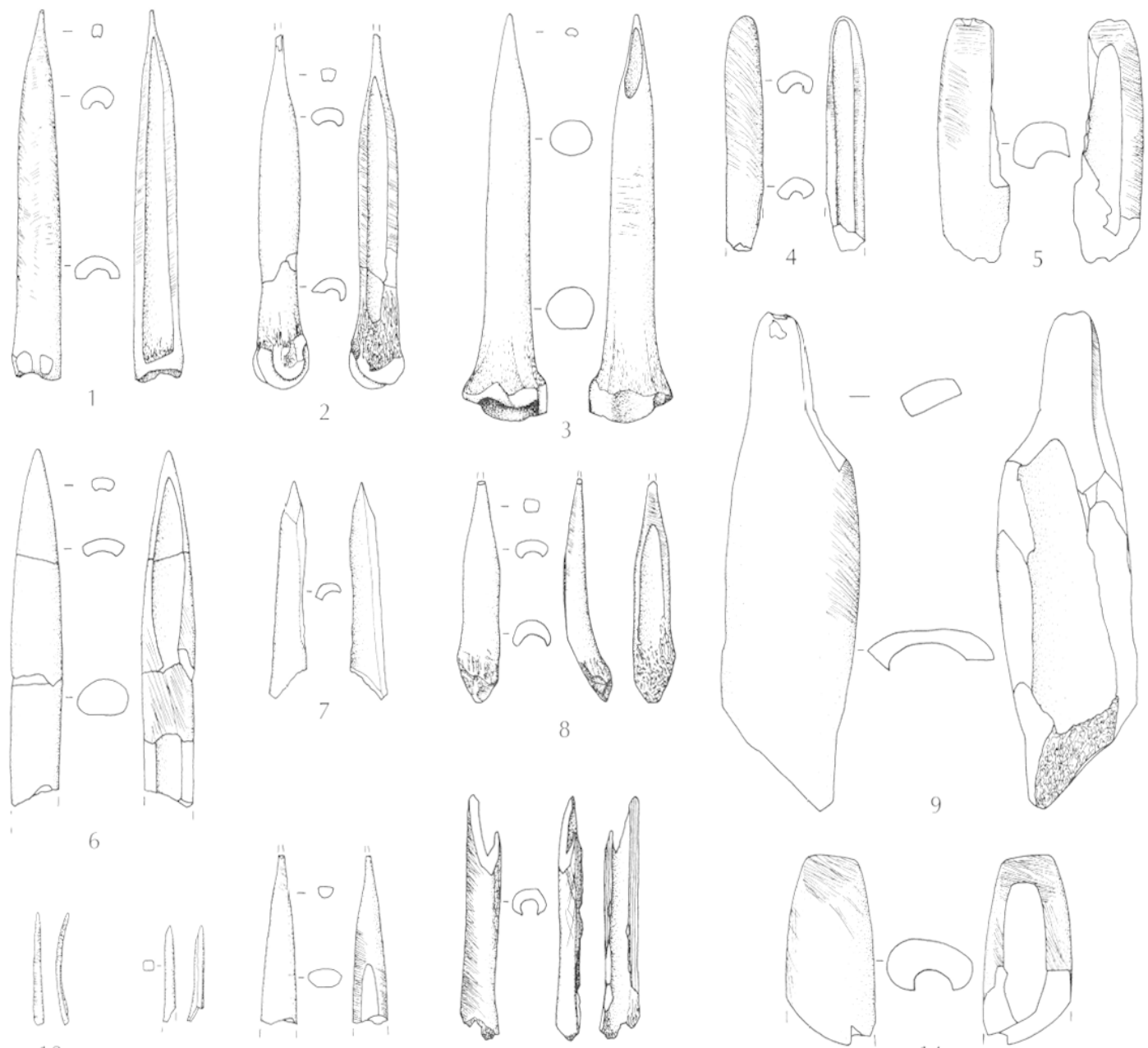

10

11

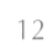

13

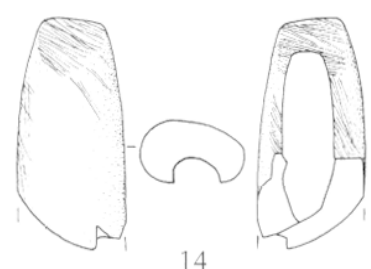

14
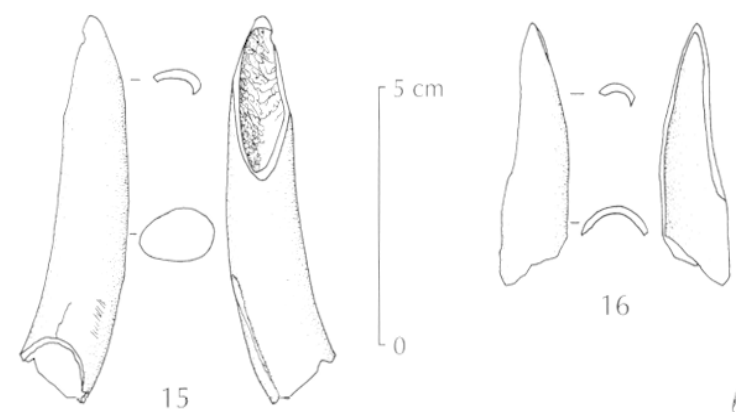

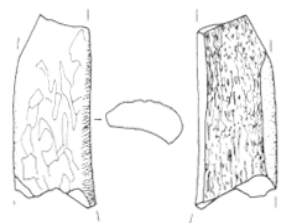

17

16

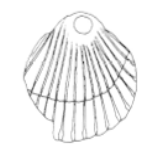

19

Fig. 11 - Industrie en matière dure animale et parure du Néolithique final (fosses, couches 2a et 2b) : 1-3, 6-12, 15, 16, pointes sur os entier ou fendu et sur esquille; 4, outil mousse; 5, 9, 14, biseau sur os fendu ou esquille; 13, pièce techrique ; 17, fragment d'outil en bois de cerf; 18 , tête d'épingle (?); 19, Cardium percé; 20, pendeloque sur canine percée; 21 , perle ovoüle épaisse en roche vert pâle; 22, perle discoïde en stéatite.

sur un tibia de lapin ; la troisième semble provenir d'un radius de capriné $\left(n^{\circ} 6\right)$, et la dernière est façonnée sur un humérus de chien $\left(\mathrm{n}^{\circ} 15\right)$. Seule la partie active de cette dernière a fait l'objet d'un aménagement particulier ; elle a été obtenue par percussion. La pointe sur un radius probable de capriné est brûlée et entièrement lus- 
trée, sa face inférieure a été aplanie par raclage. Celle prise sur un tibia présente des stries d'abrasion sur la même partie.

Cinq pointes sont tirées d'esquilles, une sur un tibia de capriné $\left(n^{\circ} 7\right)$, une autre peut-être sur un fémur de chien $\left(n^{\circ} 16\right)$, les trois autres sont indéterminées. La pointe de ces outils présente un émoussé et un lustré d'usure, le corps de ces objets n'a pas été travaillé. Une autre pointe sur un os indéterminé ( $\left.n^{\circ} 12\right)$, dont l'allure générale ressemble à celle d'une pointe fendue, pourrait en fait provenir d'une esquille entièrement façonnée.

Un fragment d'objet fin en os $\left(n^{\circ} 11\right)$, se terminant en pointe mousse, a une section quadrangulaire.

Une petite pointe très fine a été façonnée à partir d'une incisive de capriné ou de suidé $\left(\mathrm{n}^{\circ} 10\right)$. Elle présente des stries horizontales d'abrasion.

Enfin, cinq pointes ne sont représentées que par leur partie active; leur technique de débitage est indéterminable.

\section{Les outils mousses}

Un seul outil mousse est présent (fig. $11, n^{\circ} 4$ ). Il est pris sur un métapode de capriné, fendu probablement par percussion, et a perdu sa partie proximale. Sa partie active est arrondie et il est recouvert de stries obliques de façonnage.

\section{Les outils tranchants}

Les outils tranchants sont au nombre de quatre. Ils sont tous tirés d'os longs de bovinés (fig. 11).

Trois biseaux doubles sont pris sur des diaphyses d'os longs : l'un sur un fragment de tibia $\left(\mathrm{n}^{\circ} 9\right)$, le deuxième sur une esquille de tibia ou de métapode $\left(n^{\circ} 5\right)$ et le troisième sur un métapode fendu ( $\left.{ }^{\circ} 14\right)$. Le quatrième outil tranchant a perdu son extrémité distale, mais il a conservé l'amorce de sa partie active qui ressemble à celle d'un biseau. Il est tiré d'un métapode entier avec l'épiphyse. Cet outil est brûlé et très fragmenté.

Une pièce ayant perdu son extrémité distale a conservé l'amorce de sa partie active et semble également rentrer dans cette catégorie. Trois autres sont prises sur des os longs de bovinés, une sur un os long de chien, enfin la dernière n'a pas pu être déterminée.

\section{Les divers}

Un fragment mésial d'objet en bois de cerf brûlé a été fendu longitudinalement. Il montre des stries obliques de façonnage sur les deux bords (fig. 11, $\mathrm{n}^{\circ} 17$ ).

\section{LA PARURE}

La parure n'est attestée que par quatre éléments certains (fig. 11). Les perles sont représentées par un exemplaire de type cylindrique mince ou discoïde ( $\mathrm{T} 1 \stackrel{2}{2}$ of. fig. $11, n^{\circ} 22$ ), vraisemblablement en stéatite, et un autre de type ovoïde épais en roche vert pâle (T 2,cf. $\left.\mathrm{n}^{\circ} 21\right)$. La catégorie des pendeloques comporte deux éléments : une canine abrasée $\left(n^{\circ} 20\right)$, polie et percée, et un Cardium percé par abrasion $\left(\mathrm{n}^{\circ} 19\right)$.

Par ailleurs, un objet en os pourrait être une tête d'épingle $\left(n^{\circ} 18\right)$. Il est pris dans un métapode de capriné.

\section{LA CÉraMiQue}

\section{Remarques préliminaires sur le corpus}

Il n'a été remarqué, par le fouilleur, aucune évolution dans le matériel provenant de la couche archéologique, depuis le sol compacté jusqu'au sommet. Le matériel recueilli dans les fosses montre les mêmes caractéristiques que celui de la couche archéologique, ce qui est à mettre en rapport avec le fait que les fosses sont comblées par le sédiment de cette couche et ont donc vraisemblablement été réutilisées en dépotoirs. L'observation du matériel ne contredit pas cette opinion. Quelques vases provenant des prospections ou découverts hors stratigraphie ont été insérés dans le corpus lorsque ceux-ci ne laissaient aucun doute sur leur identité. L'homogénéité chronoculturelle de l'ensemble de ce matériel a donc été considérée comme le postulat de notre étude. Cependant, les intrusions ne sont pas absentes. Leur existence n'est pas très surprenante si l'on considère la fréquentation récurrente du lieu. Les plus évidentes concernent l'Âge du Bronze. Elles se présentent sous la forme de petits tessons noirs lustrés qui se situaient au sommet de la couche archéologique et ne posent

2. Dénomination d'après Hélìne Barge (1982). 
donc pas de problème particulier. Par ailleurs, nous arons été amenée à mettre en doute la datation d'une très grande coupe à bord renforcé et anse en ruban découverte au sein de la couche archéologique, au milieu d'autres fragments de grands vases (fig. 12, $n^{\circ} 1$ ). Sa pâte aux dégraissants de calcite et de calcaire très nombreux et grossiers, ses surfaces orange et granuleuses à l'extérieur, beiges à l'intérieur sont des caractéristiques très différentes de celles que nous avons décrites pour toutes les occupations mises en évidence lors de la fouille. Elles doivent être comparées aux éléments attribuables au Cardial lato sensu et récoltés en prospection à une centaine de mètres à l'ouest de La Roquette. De même, la pâte d'un tesson portant une anse en ruban mince et massive (fig. 12, $\mathrm{n}^{\circ}$ 2), à la différence de toutes les anses en ruban nettement plus larges que nous avons recensées, apparaît également conforme à celles des tessons du Néolithique ancien proche où l'on peut observer ce type d'anses.

\section{Réflexions méthodologiques}

Le manque de caractères affirmés des formes céramiques de La Roquette se pose comme une contrainte lorsqu'il s'agit d'établir un classement et nécessite une réflexion sur la fonctionnalité d'une tỵpologie.

Les différents sustèmes de classement ont pour finalité soit de reconnaître, dans une zone d'étude particulière, des composantes culturelles issues de diverses régions géographiques, soit de discerner, au sein d'une même culture, des phases chronologiques et des faciès culturels, soit de démêler, dans une seule série, ce qui peut appartenir à une culture de ce qui est redevable à une autre. Pour nous, il s'agit simplement de permettre d'établir des comparaisons qui ne peuvent être que limitées. En effet les ensembles céramiques du Néolithique final méridional ne sont guère plus caractérisés que ceux de La Roquette, du moins en ce qui concerne les formes. Seules des analyses quantitatives de ces séries à travers la publication exhaustive des mobiliers pourraient autoriser des comparaisons précises, mais cette approche méthodologique a rarement été mise en cuure. Elles seules permettent pourtant d'avancer de manière significative dans les approches chronotypologiques.

Nous arons tenté d'éviter le "manque de précision [qui] tient en partie au fait que les chercheurs oscillent
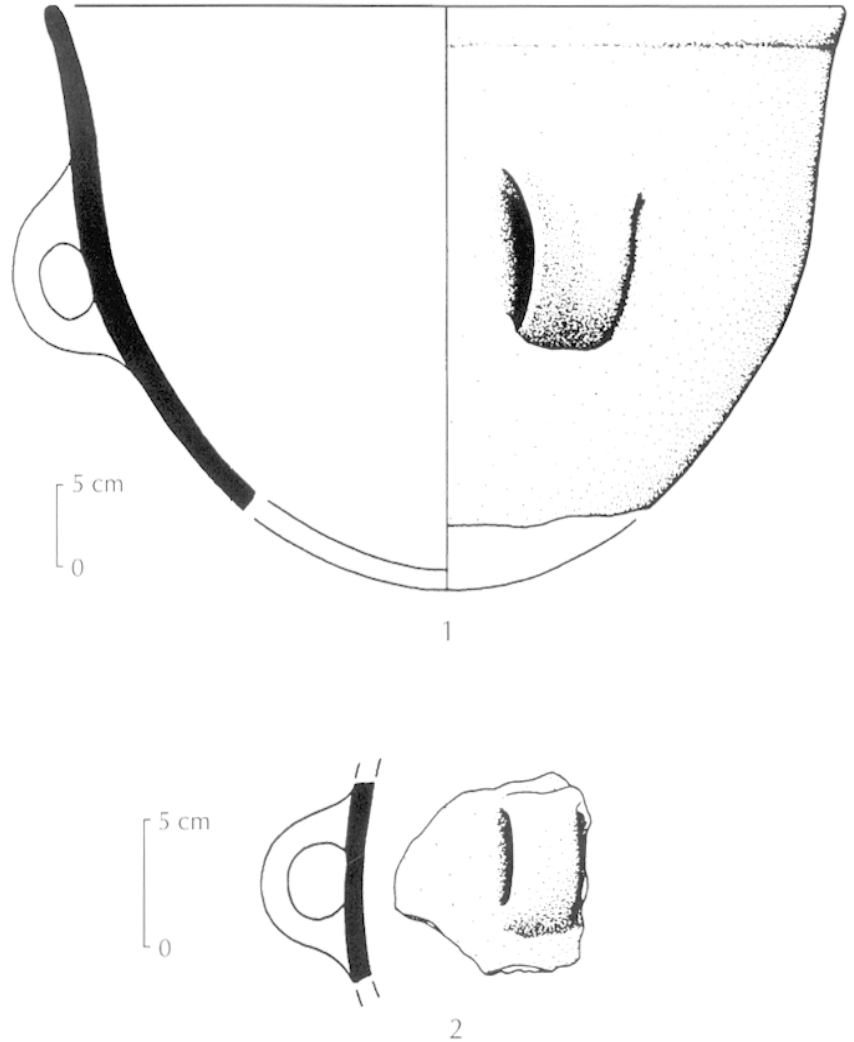

Fig. 12 - Céramique probablement intrusive, récoltée dans la couche 2 a (Néolithique ancien?) : 1, grand vase hémisphérique à anse en ruban; 2, tesson portant une anse en ruban.

entre des descriptions "fonctionnelles" et des descriptions purement "formelles" "(Gallay, 1977, p. 44). Le parti pris est celui d'une typologie fondée sur des critères morphologiques; les critères de proportions intervenant essentiellement pour distinguer les jarres profondes. D'autre part, nous avons préféré nous en tenir aux termes de "coupe " et " jarre " pour désigner les formes qui restent trop peu différenciées pour être distinguées sur le plan fonctionnel. Les deux termes quc nous avons choisis ont acquis, chez les néolithiciens, un sens beaucoup plus large que leur définition primaire.

La typologie établie par Xavier Gutherz (1984) pour le Ferrières nous a semblé suffisante, compte tenu de ce que nous avions à démontrer. Cet auteur distingue des formes géométriques simples dérivées de la sphère et de l'ellipse, et des formes issues de la combinaison de deux volumes (sphère/cylindre, ellipse/cône, etc.). Du point de vue fonctionnel, nous distinguons schématiquement, en accord avec cet auteur, les vases à boire et à manger 
(coupes), les récipients destinés au transfert (jarres fermées) et ceux réservés à la conservation, au stockage (jarres profondes). L'adoption de ce système de classement nous permet de comparer directement l'ensemble de La Roquette avec ceux du Ferrières avec lesquels les affinités sont les plus fortes, en employant le même langage descriptif.

\section{Répertoire des formes céramiques}

Groupe 1. Coupes en calotte de sphère ou d'ellipsoïde horizontale ou verticale (fig. 13) : les parois sont subrectilignes et nettement divergentes.

Groupe 2. Coupes à fond hémisphérique ou en demiellipsoïde et à paroi redressée formant un changement de direction plus ou moins prononcé avec le fond (fig. 14) : ce sont des vases combinant deux volumes géométriques simples. Les parois sont cylindriques, tronconiques ou tronconiques inverses. Cette catégorie montre une grande disparité dans le rapport hauteur/largeur. Elle comprend quelques pots dont le profil est proche des vases à fond bombé et paroi dégagée de Jean Vaquer (1975). Il s'agit essentiellement des coupes dont le diamètre est au moins égal au double de la hauteur estimée mais qui ne montrent pas de carène franche. La rupturc de pente de deux d'entre elles est marquée par un léger amincissement de l'épaisseur de la paroi.

Groupe 3. Coupes hémisphériques ou en demi-ellipsoïde (fig. 15) : ce sont des vases à paroi courbe ne dépassant pas la demi-sphère ou la demi-ellipse. Sur certains exemplaires, le bord est marqué par une contre-courbe. Dans cette catégorie, un vase $\left(n^{\circ} 16\right)$ se démarque nettement des autres par ses très grandes proportions $(38 \mathrm{~cm}$ de diamètre pour $29,5 \mathrm{~cm}$ de profondeur).

Groupe 4. Coupes sphéroïdales ou ellipsoïdales à grand axe horizontal (fig. 16) : la paroi dépasse la demi-sphère ou la demi-ellipse.

Groupe 5. Jarres sphéroïdales ou ovoïdes (fig. 17) : elles sont définies par une embouchure plus ferméc que les coupes sphéroïdales et/ou une plus grande profondeur. Pas plus que pour les coupes, la profondeur de ces vases ne dépasse le diamètre maximal. Bien que le volume principal soit toujours la sphère ou l'ove, on note des exemplaires à profil sinueux, à paroi légèrement biconique ou à paroi courte tronconique.

Groupe 6. Jarres profondes à fond hémisphérique ou $\mathrm{cn}$ demi-ellipsoïde et à paroi cylindrique, tronconique et tronconique inverse (fig. 18) : ce sont les seuls vases de notre série dont la profondeur estimée dépasse le diamètre maximal. Dans tous les cas, ils combinent deux volumes géométriques simples.

Le rapport entre le diamètre et la profondeur estimés des vases donne une concentration autour de $10-30 \mathrm{~cm}$ de diamètre maximal et autour de $5-15 \mathrm{~cm}$ de profondeur. Un pic net à $10-15 \mathrm{~cm}$ de diamètre et $5-10 \mathrm{~cm}$ de profondeur correspond essentiellement aux coupes hémisphériques. Lcs dimensions des jarres profondes, que l'on a rarement pu estimer, se situent autour de $35-50 \mathrm{~cm}$ de diamètre et $40-50 \mathrm{~cm}$ de profondeur. Rappelons ici la présence d'une grande coupe dont le diamètre $(38 \mathrm{~cm})$ est plus proche de celui des jarres profondes.

L'histogramme de la structure morphologique de la céramique (fig. 19), élaboré à partir de 87 formes identifiables, fait apparaître la nette prédominance des coupes en calotte de sphère ou d'ellipse et des coupes sphéroïdales ou ellipsoïdales (respectivement : $21,8 \%$ et $22,9 \%)$; les jarres profondes sont les moins nombreuses $(5,7 \%)$. Les formes basses en général (types 1 à 3$)$ représentent $55 \%$ du stock, et les formes hautes et fermées (types 5 et 6 ), $21,7 \%$. Le type 4 (coupes sphéroïdales, $22,9 \%$ ) peut être considéré comme un intermédiaire entre ces deux groupes.

Les lèvres se situent généralement dans le prolongement de la panse; cependant on note également la présence sporadique de lères infléchies vers l'extérieur des récipients (contre-courbes) sur des rases de petites et moyennes dimensions. Elles sont en majorité arrondies mais celles qui sont ogivales ou impressionnées sont également bien présentes. On trouve quelques types biscautés, aplatis et bourrelés. Signalons que la moitié des impressions qui orne les lèvres nous semble réalisée au doigt; certaines portent les marques caractéristiques de l'ongle (fig. 22). Nous n'avons identifié qu'une seule lèrre qui réponde à la définition de "crénelée " : application sur la lève d'un instrument tranchant ou mousse perpendiculairement à la paroi du vase (Jacobieski, 1988). Pour les autres, la technique utilisée n'a pas pu être identifiée. 

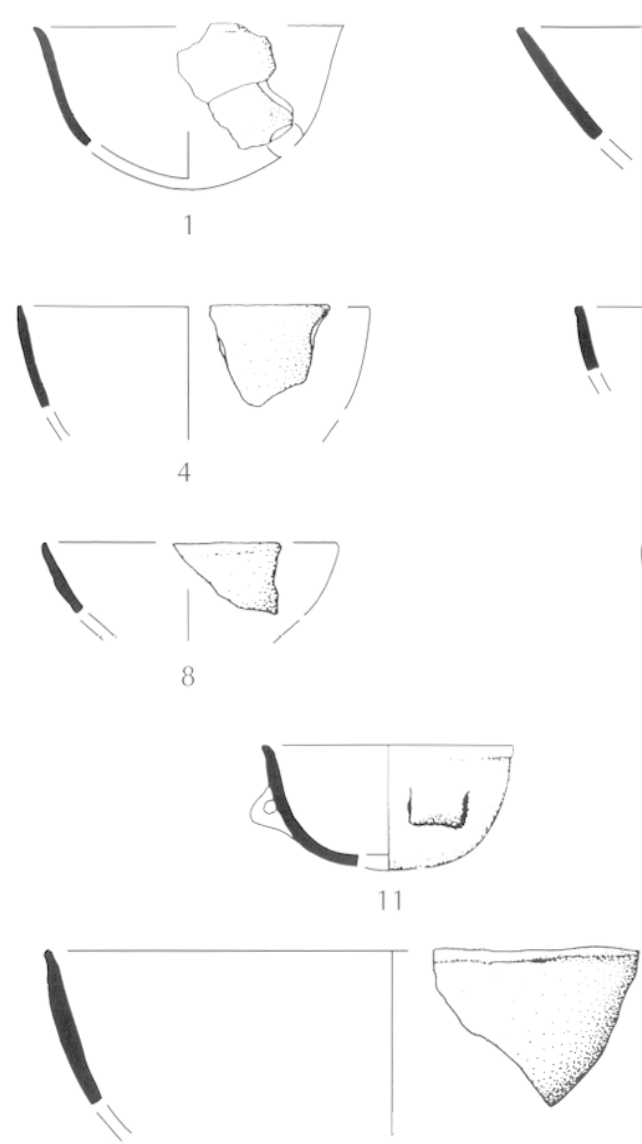

13

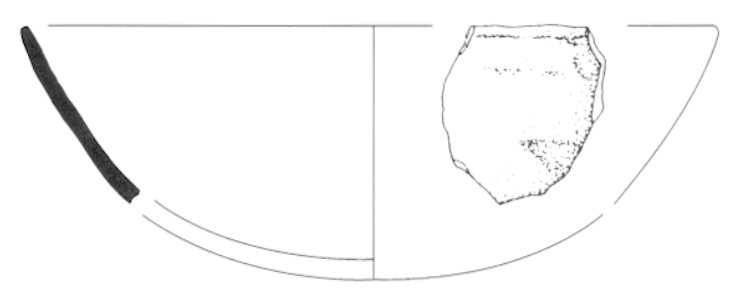

15
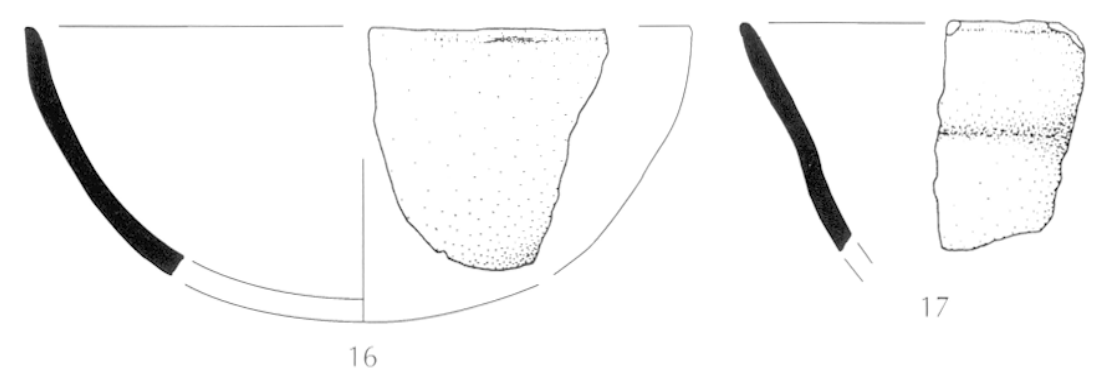

17

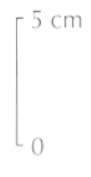

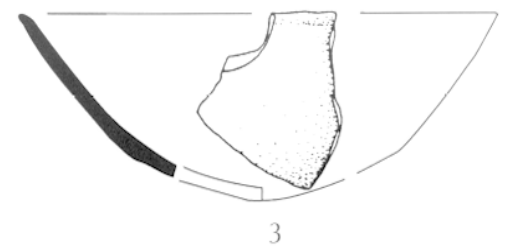
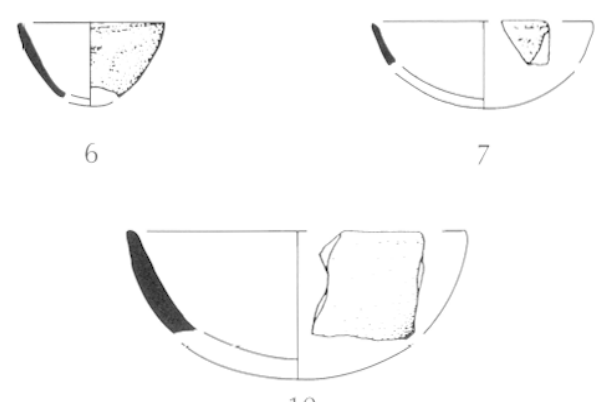

10

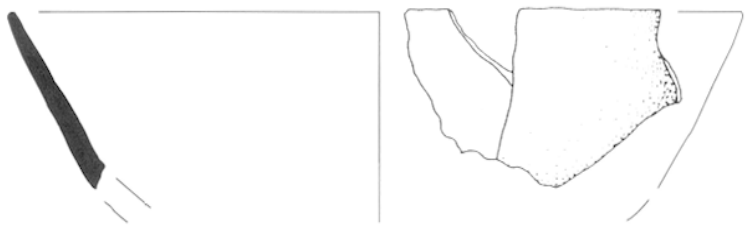

12
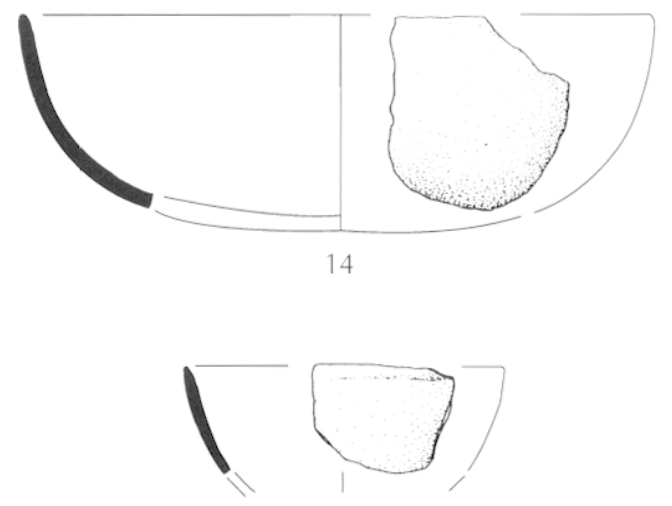

18

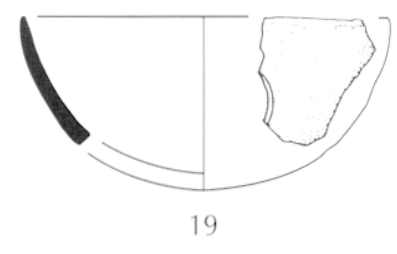

Fig. 13 - Céramique du. Véolithique final, groupe 1 (fosses, couches 2a et 2b) : coupes en calotte de sphère ou d'ellipsoïle. 

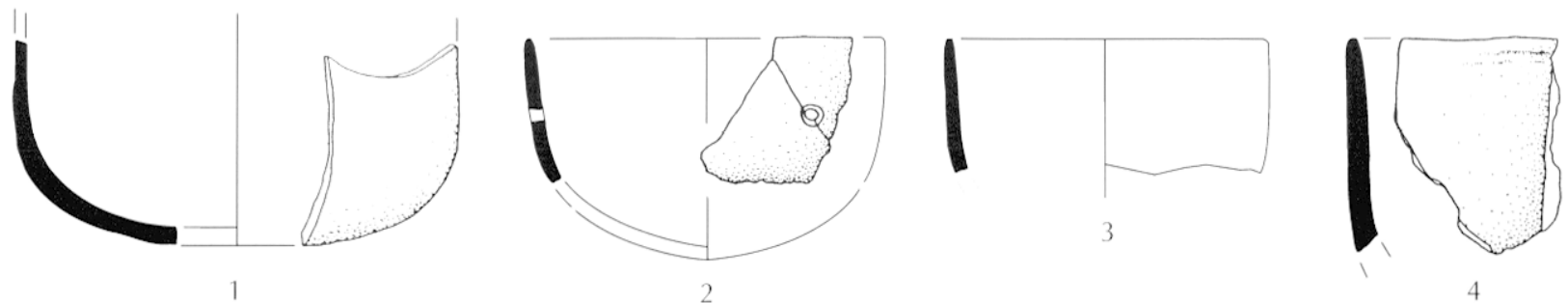

4
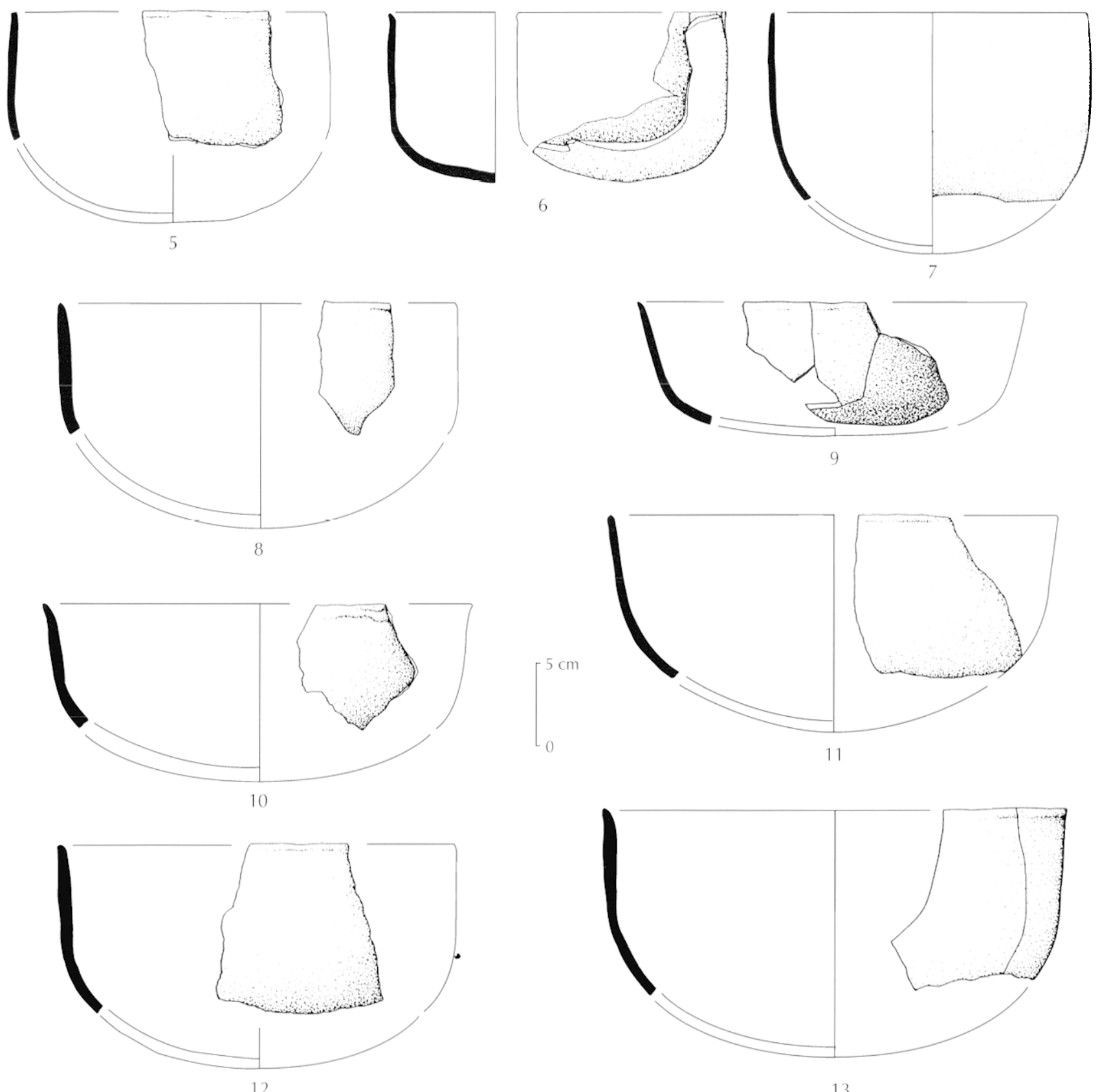

13

Fig. 14 - Céramique du Néolithique final, groupe 2 (fosses, couches $2 a$ et 2b) : coupes à fond hémisphérique ou en demi-ellipsoüde et à paroi redressée. 

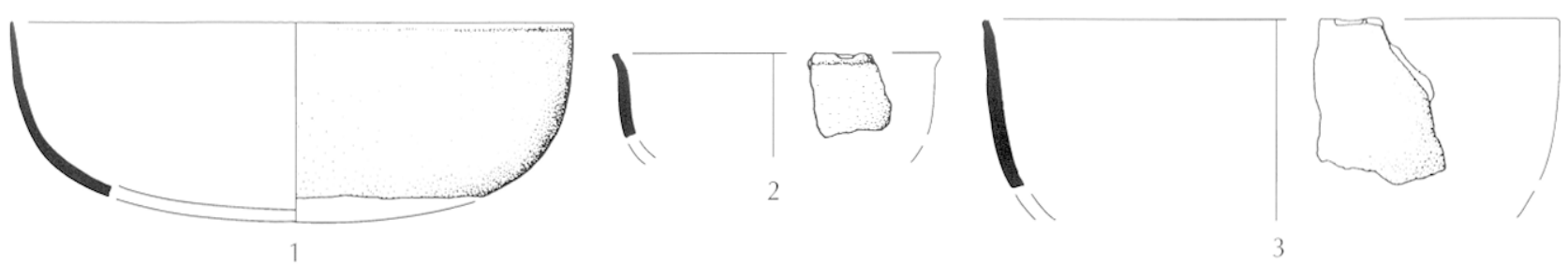

।
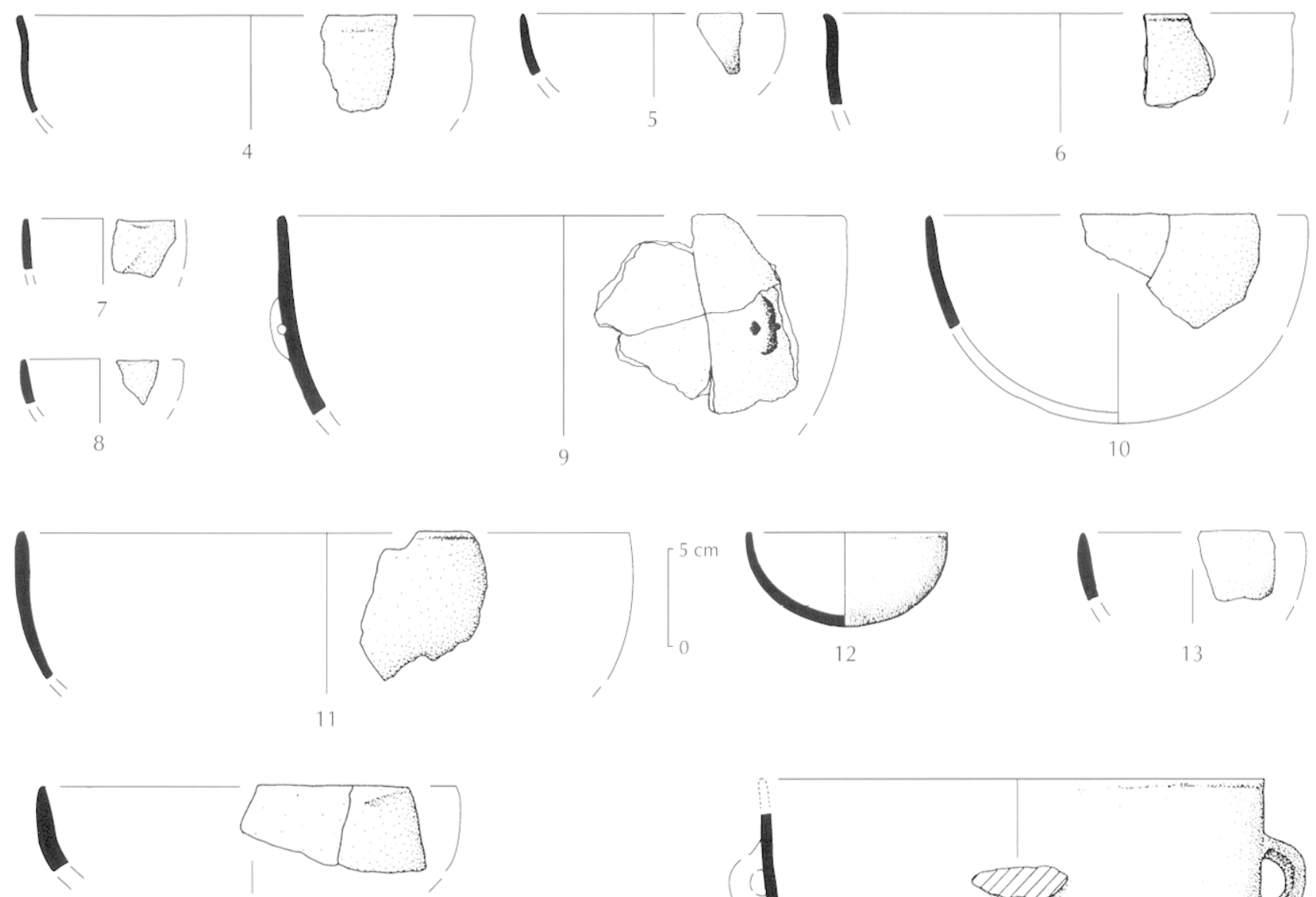

14
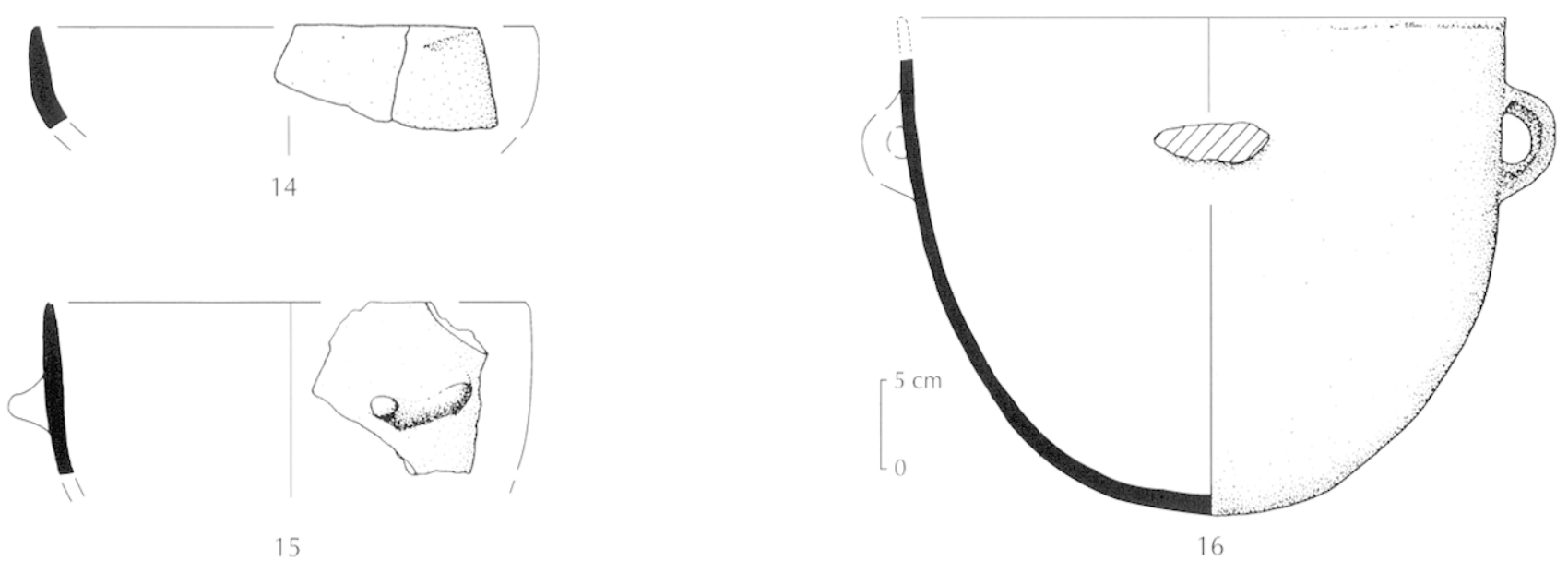

15

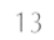

Fig. 15 - Céramique du Néolithique final, groupe 3 (fosses, couches $2 a$ et 2b) : coupes hémisphériques ou en demi-ellipsoïde. 

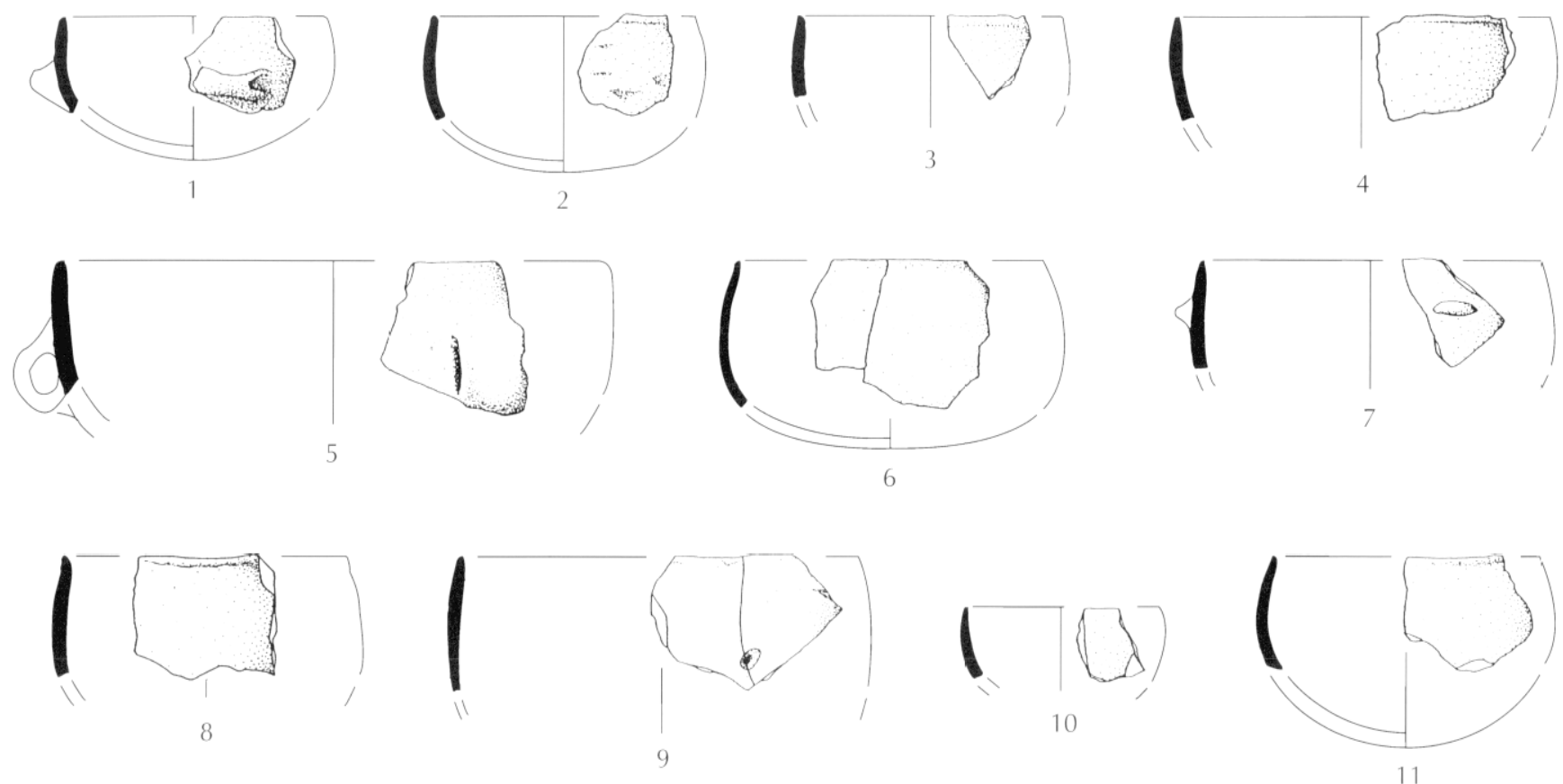

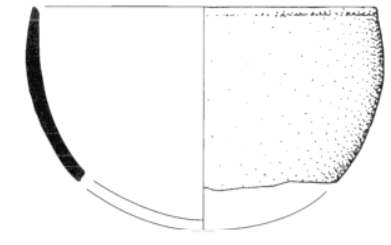

12

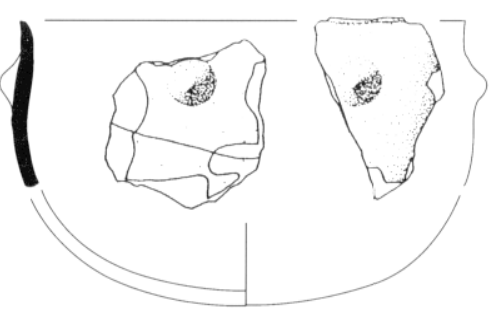

16

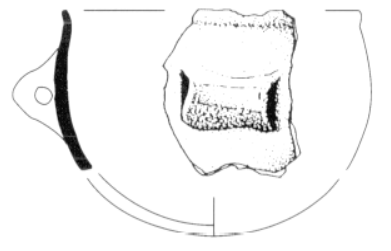

13
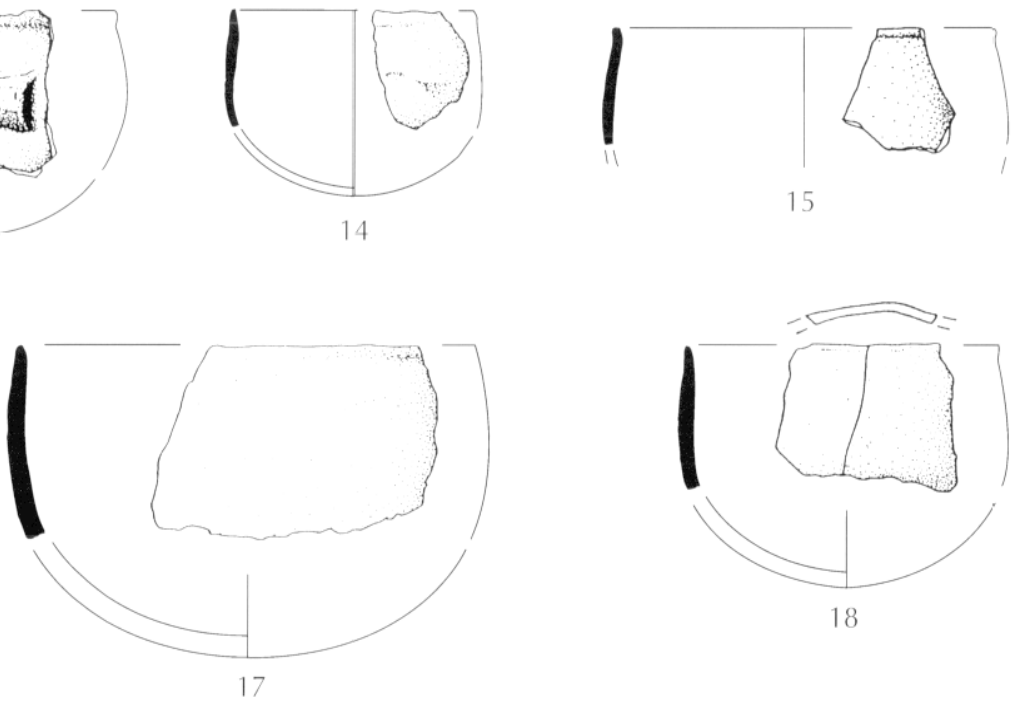

15

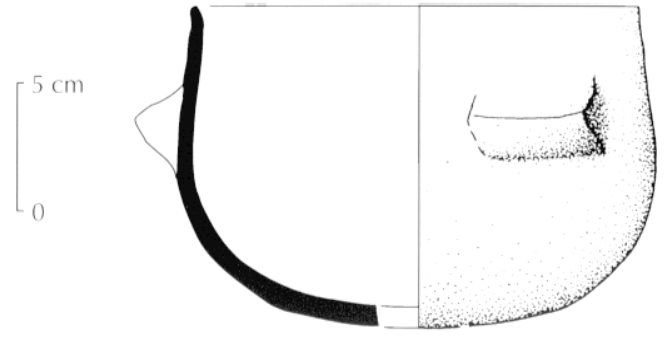

20

Fig. 16 - Céramique du Néolithique final, groupe 4 (fosses, couches $2 a$ et 2b) : coupes sphéroïdales ou ellipsoïdales. 

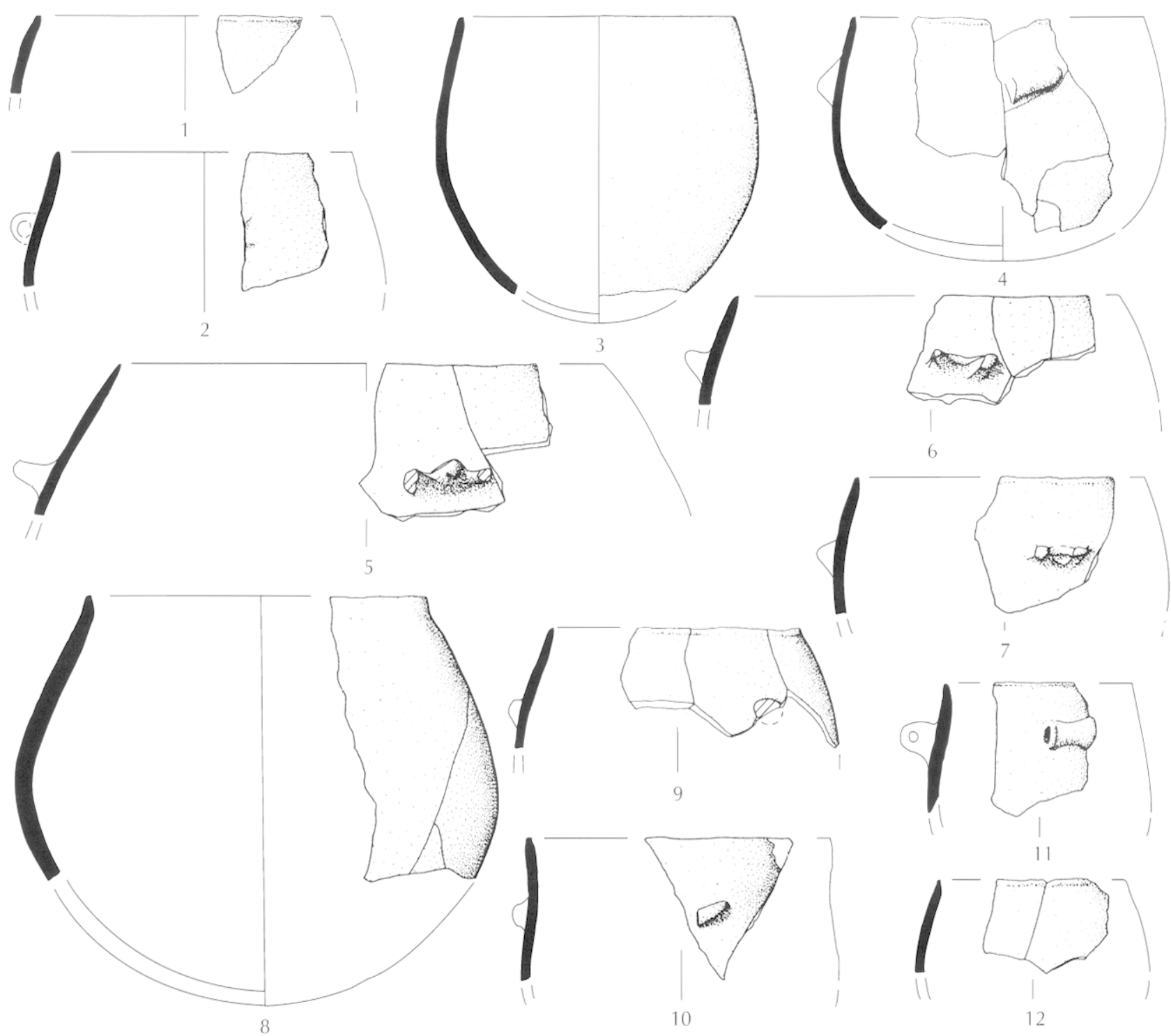

10

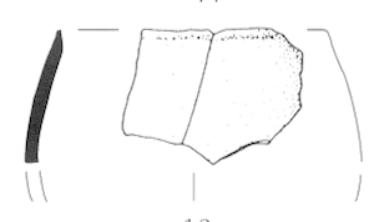

12
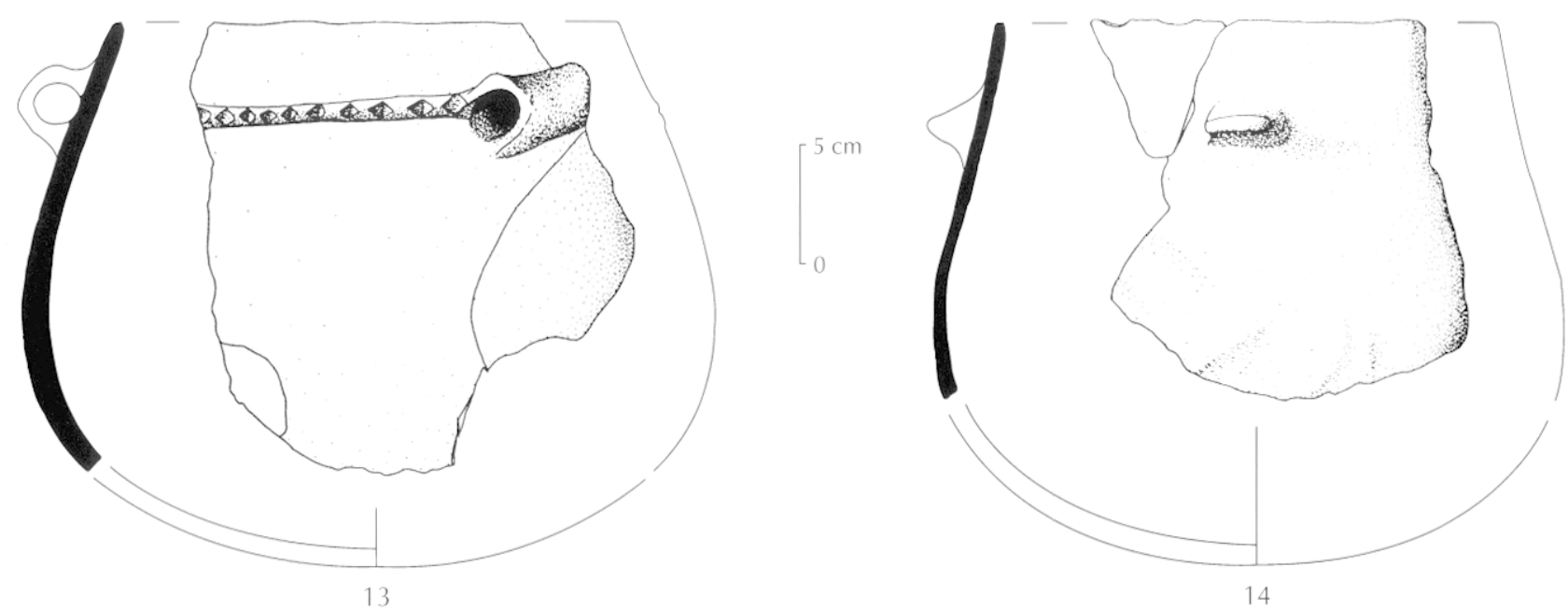

Fig. 17 - Céramique du Néolithique final, groupe 5 (fosses, couches $2 a$ el 2b) : jarres sphéroüdales ou ovoüdes. 
276

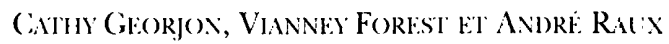
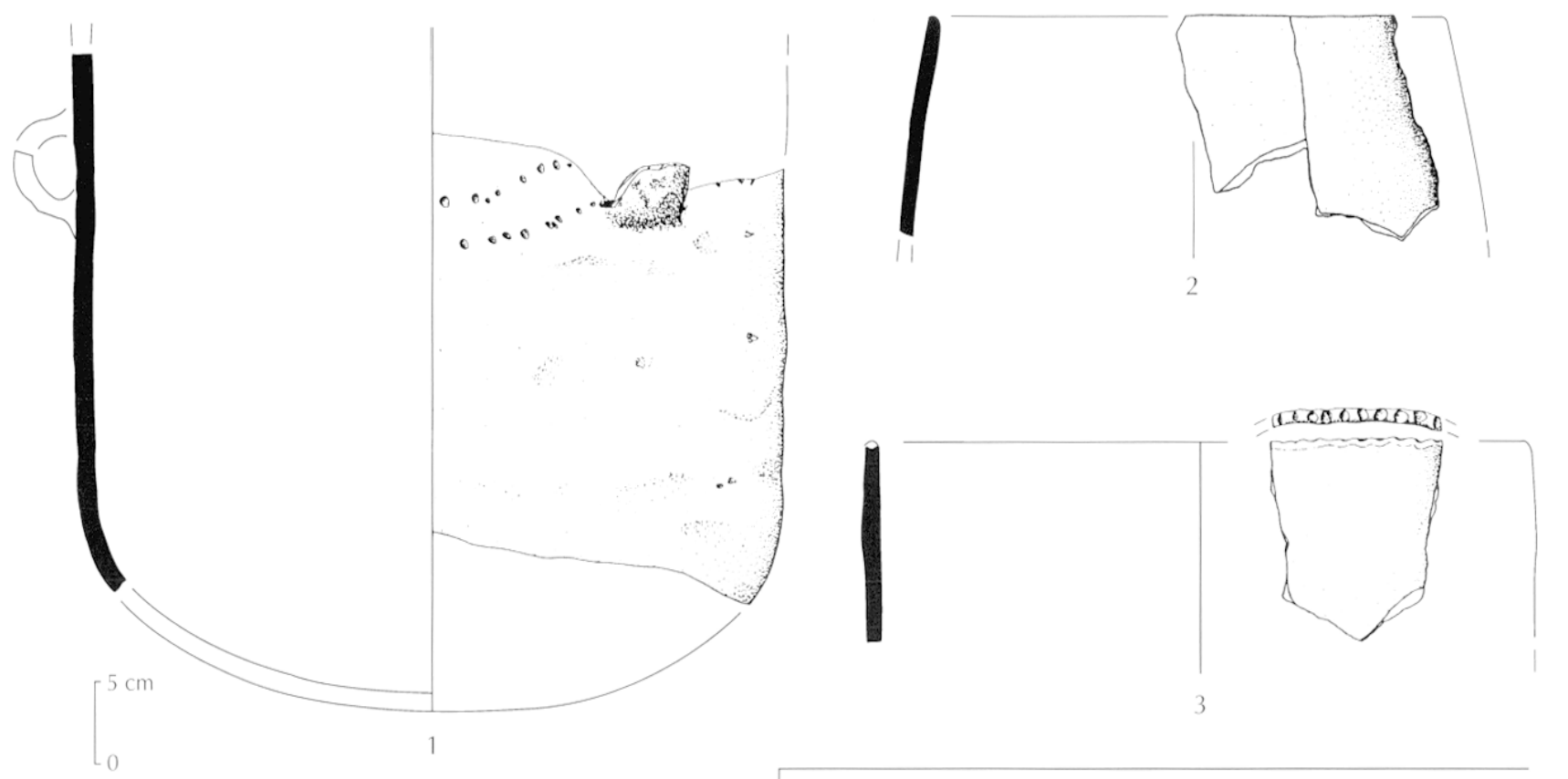

3

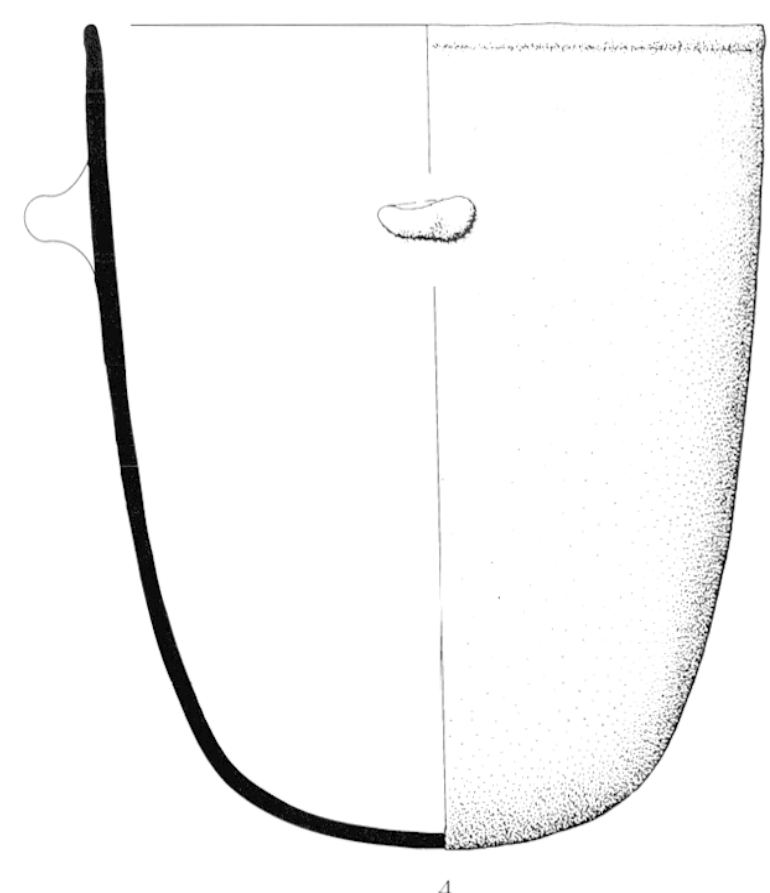

4

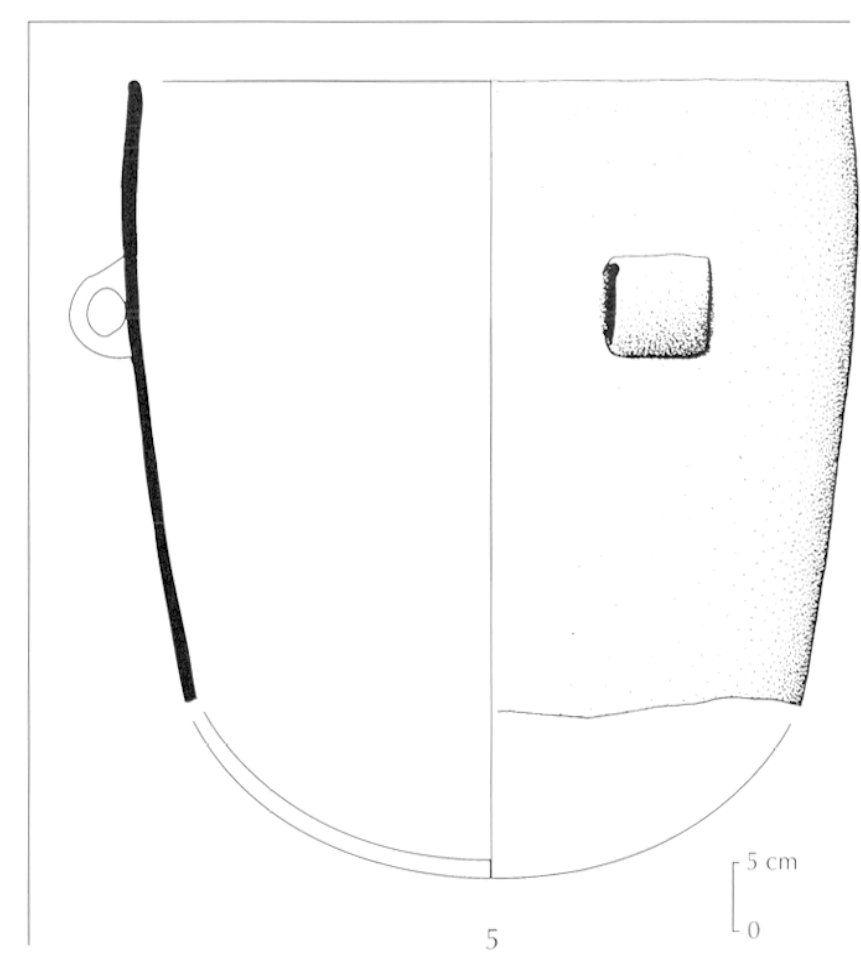

5

Fig. 18 - Céramique du Néolithique final, groupe 6 (fosses, couches 2a et 2b) : jarres profondes à fond hémisphérique ou en demi-ellipsoïde et à paroi cylindrique, tronconique ou tronconique inverse.

Gallia Prathistoire, 41, 19999, p. 253-297

(1) CNRS F́DITIONS. Paris 2000 


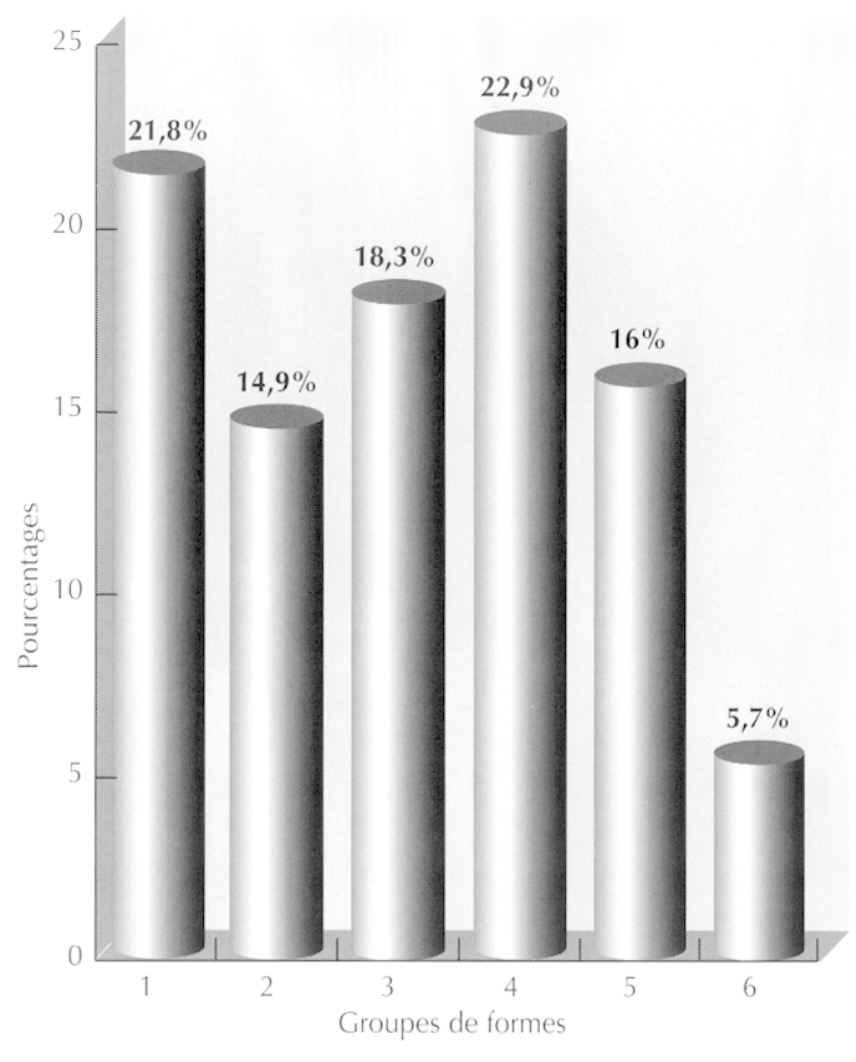

Fig. 19 - Histogramme des groupes morphologiques de la céramique du Néolithique final de La Roquette.

\section{Répertoire des éléments de préhension}

Les éléments de préhension sont nombreux et variés à La Roquette. La typologie utilisée est fondée sur différentes publications concernant des ensembles aussi bien ferrériens (Gutherz, 1984 ; Jacobieski, 1988) que couronniens (Montjardin, 1984).

Les préhensions ont rarement été mises en évidence sur les formes basses de types 1 à 4 ; la majorité est donc issue des formes hautes et fermées. Elles sont toujours situées dans la partie supérieure des vases, souvent sur lc haut de la panse, excepté pour de très petits vases dont l'élément de préhension monopolise toute la hauteur de la paroi. Leur disposition est toujours horizontale, excepté dans deux cas : une prise semi-cylindrique oblique et une oreille (fig. $15, \mathrm{n}^{\circ} 9$ et fig. $17, \mathrm{n}^{\circ} 10$ ).

Tous les éléments de préhension sont appliqués sur la paroi des rases préalablement creusée.

1. Boutons ronds (fig. 20, $\mathrm{n}^{\circ 1} 1-3$ ).

2. Languettes : elles peuvent être simples (fig. $20, \mathrm{n}^{\text {"N }} 7-9$, $10,14,17,18,21-23$ ), ensellées (fig. 20, $n^{\circ} 4$ ), bilobées (fig. 20, $\mathrm{n}^{\circ} 27,30,33,36,39$ ), trilobées (fig. 20, $\mathrm{n}^{\circ} 26$, 37) ou à double impression (fig. $23, \mathrm{n}^{\circ} 12$ ). Dans ce dernier cas, elles présentent trois protubérances qui les assimilent aux languettes trilobées.

3. Oreille perforée (fig. 15, $n^{\circ} 9$ ).

4. Anses en ruban : elles sont simples (fig. 20, $\mathrm{n}^{\circ \mathrm{N}} 19,40$ ) ou ensellées (fig. $17, \mathrm{n}^{\circ} 13 \mathrm{et} \mathrm{fig.} 20, \mathrm{n}^{\circ} 35$ ) et présentent la particularité d'être la plupart du temps aussi larges que hautes.

5. Prises semi-cylindriques : on rencontre des types non perforés (fig. 17, $\mathrm{n}^{\circ} 4$ ), perforés (fig. 20, $\mathrm{n}^{\mathrm{N}} 11,15,20,24$, 38), pseudo-funiculaires (enfoncement des deux côtés de la prise pratiqué au doigt ou début de perforation, fig. 20, $n^{\circ} 25$ ) ou en «pseudo-H» (prolongement de la partie supérieure de la prisc par deux cordons verticaux, fig. 20, $n^{\circ} 32$ ).

6. Bobines : elles se distinguent des prises semi-cylindriques par une dépression centrale du fût. Elles sont perforées (fig. 20, $\mathrm{n}^{\mathrm{N}} 5,6,12,28,29,34$ ), pseudo-funiculaires (fig. $16, \mathrm{n}^{\circ} 1$ et fig. 20, $\mathrm{n}^{\circ} 31$ ) ou à renflement médian vertical (en "nœud papillon ", fig. 20, n 16).

7. Prises prismatiques de section triangulaire (fig. 16, $\left.\mathrm{n}^{\circ} 20\right)$.

L'histogramme de la structure morphologique des préhensions a été réalisé à partir de 69 éléments (fig. 21). Il montrc quc les languettes sont les éléments de préhension les plus courants (41\%), suivies des anses en ruban $(27 \%)$ et des bobines $(17 \%)$. Dans les soustypes, on remarque la bonne représentation des anses en ruban simples $(24 \%)$, des languettes simples $(20 \%)$, des languettes bilobées (11\%) et des bobines simples perforées $(11 \%)$. Les anses en ruban ensellées et les languettes trilobées ne représentent que $3 \%$ chacune. Les autres sous-types ne sont présents qu'à un ou deux exemplaires.

\section{Répertoire décoratif}

Les décors sont très rares. Sur plus de 3000 tessons, nous n'en avons recensé que 47 . La typologie que nous utilisons est celle de la thèse de Xavier Gutherz (1984).

1. Incisions : 7 tessons (fig. $22, \mathrm{n}^{\circ \mathrm{s}} 17,19-23,25$ ) ; elles sont soit profondes en portée longue verticale ou horizontale ou en portée verticale courte, soit très légères en tirets obliques et portée longue. Dans un cas, elles se développent autour d'une anse en ruban et, dans un autre cas, elles sont près d'un bouton rond. 

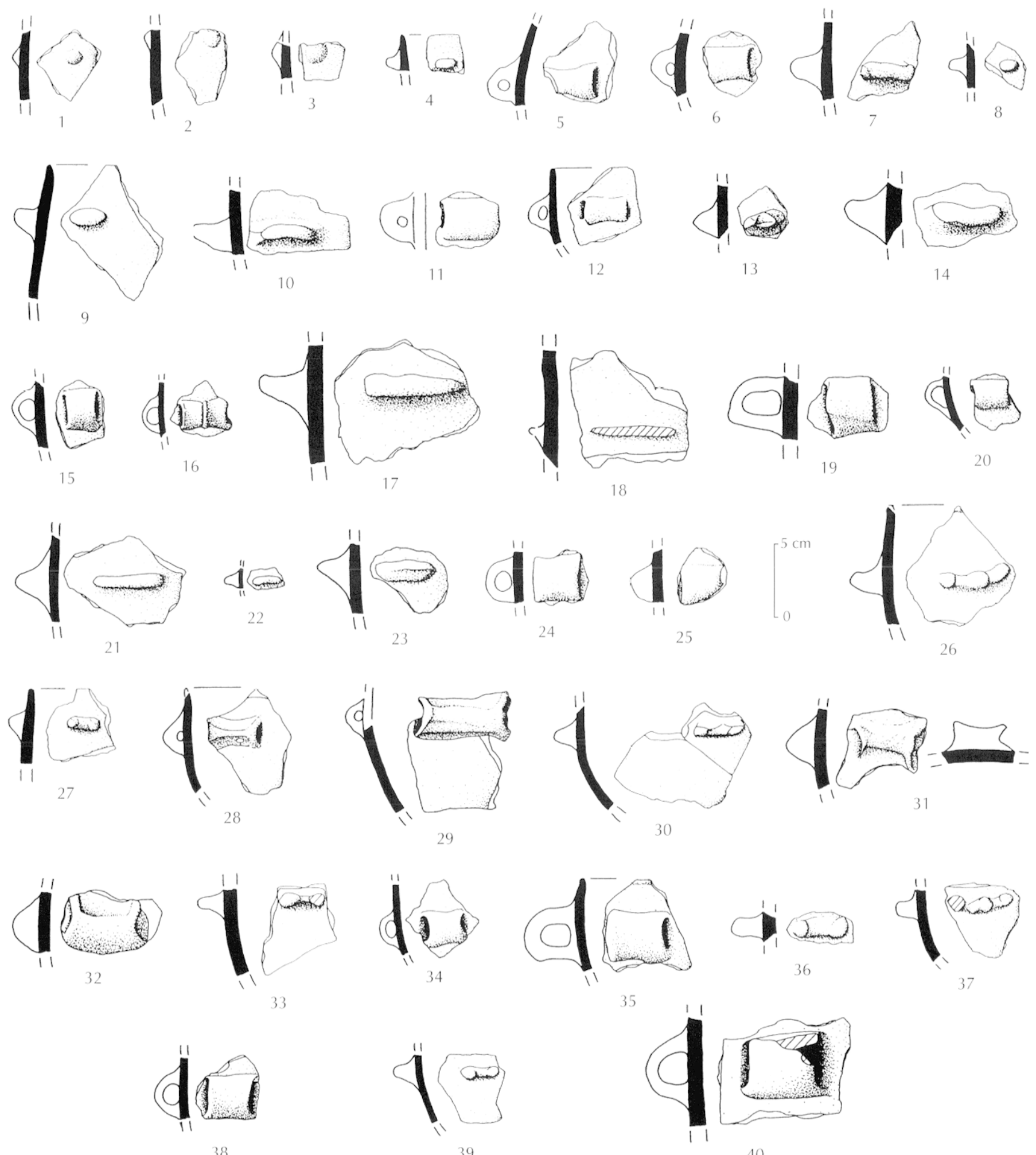

Fig. 20 - Céramique du Néolithique final, éléments de préhension (fosses, couches 2 a et 2b) : 1-3, boutons ronds ; 4, 7-9, 10, 14, 17, 18, 21-23, languettes simples; 5, 6, 12, 28, 29, 34, bobines perforees ; 11, 15, 20, 24, 38, prises semi-clindriques ferforées; 13, 26, 27, 30, 33, 36, 37, 39, languetles bilobies ou trilobées; 16, prise à renflement médian; 19, 35, 40, anses en ruban simples ou ensellées; 25, 31, prise pseudofuniculaire; 32, prise en "pseudo-H". 


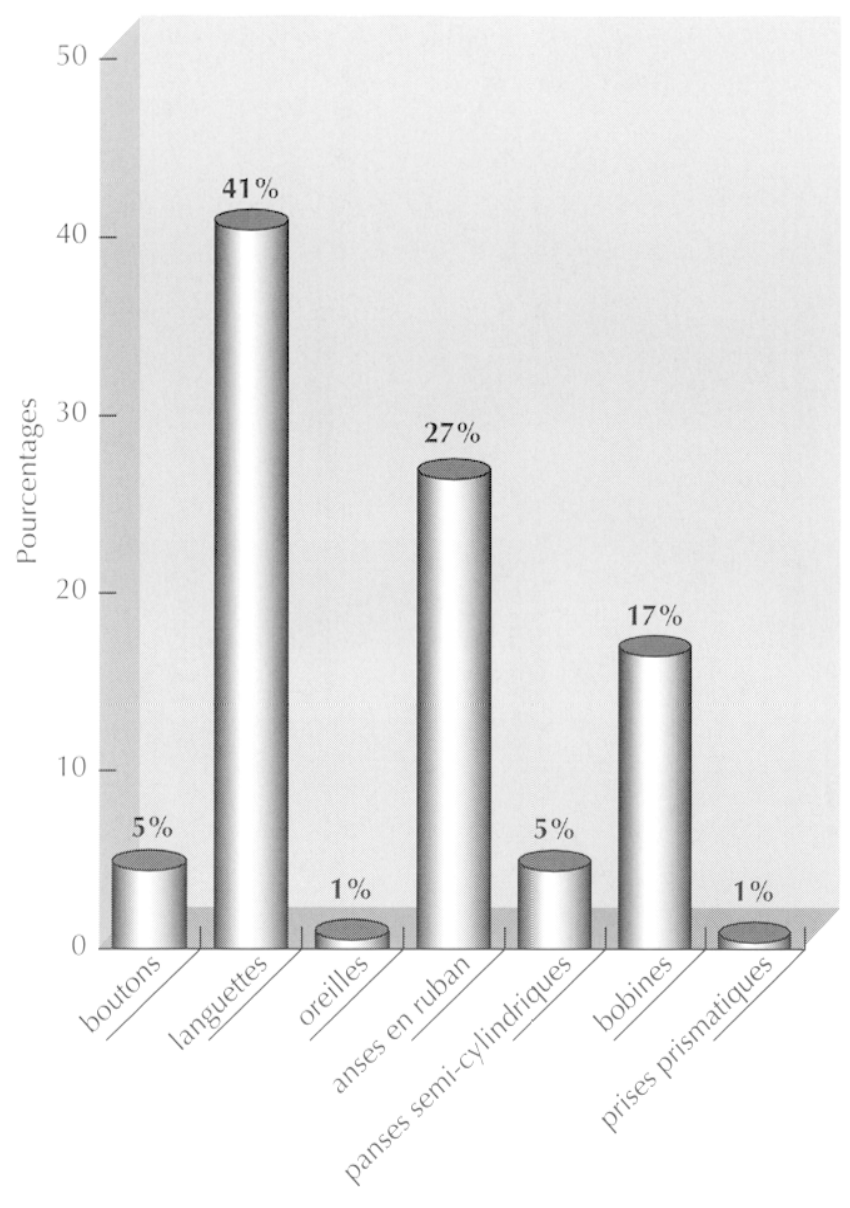

Fig. 21 - Histogramme des éléments de préhension du Néolithique final de La Roquette.

2. Cannelures : 1 tesson (fig. 22, $n^{\circ} 23$ ) ; elles sont larges et très légères en chevrons jointifs simples.

3. Impressions : 17 sur panse et 18 sur lèvre (fig. $22, n^{\circ s} 1$ $15,26,27)$; elles sont subcirculaires irrégulières, ovales, en " graines de melon ", subcarrées ou triangulaires, organisées en portée longue horizontale ou en guirlande. Certaines semblent digitées.

4. Cordons impressionnés : 2 tessons (fig. $17, \mathrm{n}^{\circ} 13$ et fig. 22, $\mathrm{n}^{\circ} 28$ ).

5. Pastilles appliquées : 2 tessons (fig. $16, n^{\circ} 16$ et fig. 22 , $\left.n^{\circ} 16\right)$.

Les décors à La Roquette n'atteignent donc pas 1,5\% du total des tessons. Les impressions y sont très largement dominantes. Tous les décors sont simples; aucun n'est composé (associant au moins deux thèmes exécutés avec la même technique) ou complexe (associant au moins deux techniques différentes).

\section{Les caractéristiques techniques}

Les observations techniques ont été réalisées à l'œil nu; elles ne sont donc que très générales. F́tant donné que seules les études à la loupe binoculaire semblent autoriser l'emploi de termes spécifiques servant à décrire les états de surfaces, tels que " raclage ", "lissage " ou "brunissage ", etc. (Binder et al., 1994), nous ne parlerons ici que de "surfaces mates " et de "surfaces brillantes".

L'ensemble des tessons montre une grande homogénéité sur le plan technique. Excepté pour les jarres profondes, on ne discerne pas de différence de pâte et de traitement selon les catégories de vases.

La cuisson est bonne la plupart du temps, car la céramique est dure et sonore. Sur cassure fraîche, la structure de la pâte apparaît presque toujours la même, elle est assez compacte et grenue, plus rarement feuilletée. La couleur est généralement uniforme sur cassure. Les grains de dégraissant, bien répartis, apparaissent en grand nombre sur une cassure fraîche. Ils se composent essentiellement de calcite broyée et de calcaire. En général, leur taille est voisine du millimètre avec des inclusions d'éléments plus gros ne dépassant que rarement $3 \mathrm{~mm}$, sauf pour les grands vases (diamètre supérieur à $30 \mathrm{~cm}$ ). La coulcur des surfaces varic du beige (trc̀s rarc) au rose et de l'orangé au noir en passant par le brun moyen et le gris foncé. Les teintes foncées sont les plus fréquentes (gris foncé surtout). Pour les grands vases (diamètre supérieur à $30 \mathrm{~cm}$ ), les teintes orangées et gris foncé sont les plus répandues. L'épaisseur des parois est en général en accord avec le volume des vases. L'aspect des surfaces est assez souvent bosselé et rugueux au toucher (dégraissants) sauf pour plusieurs jarres profondes dont la surface est plutôt mate et poudreuse, ce qui peut indiquer une cuisson moins poussée. Des traces d'estèque sont parfois visibles, notamment sur les grandes jarres profondes. Notons des traces grossières d'estèque sur la paroi intérieure de quelques coupes et sur une grande jarre profonde dont la surface est très irrégulière et semble présenter des coups d'ongle. La lèvre est, dans de nombreux cas, marquée par un léger sillon d'outil discontinu et sinueux. De petites zones de brillance sont présentes sur de nombreux tessons, aux parois irrégulières ou régulières, que les dégraissants soient apparents ou non. Quelques fragments montrent des surfaces très rugueuses dues à une altération. 
Quelques vases, essentiellement des coupes, ont une paroi très mince $(4-5 \mathrm{~mm})$ par rapport à leur volume. Leur paroi, uniformément gris foncé ou noire, présente une brillance uniforme à travers laquelle on devine les traces laissées par l'outil. Quelques dégraissants sont cependant visibles en surface et la paroi n’a pas une régularité parfaite.
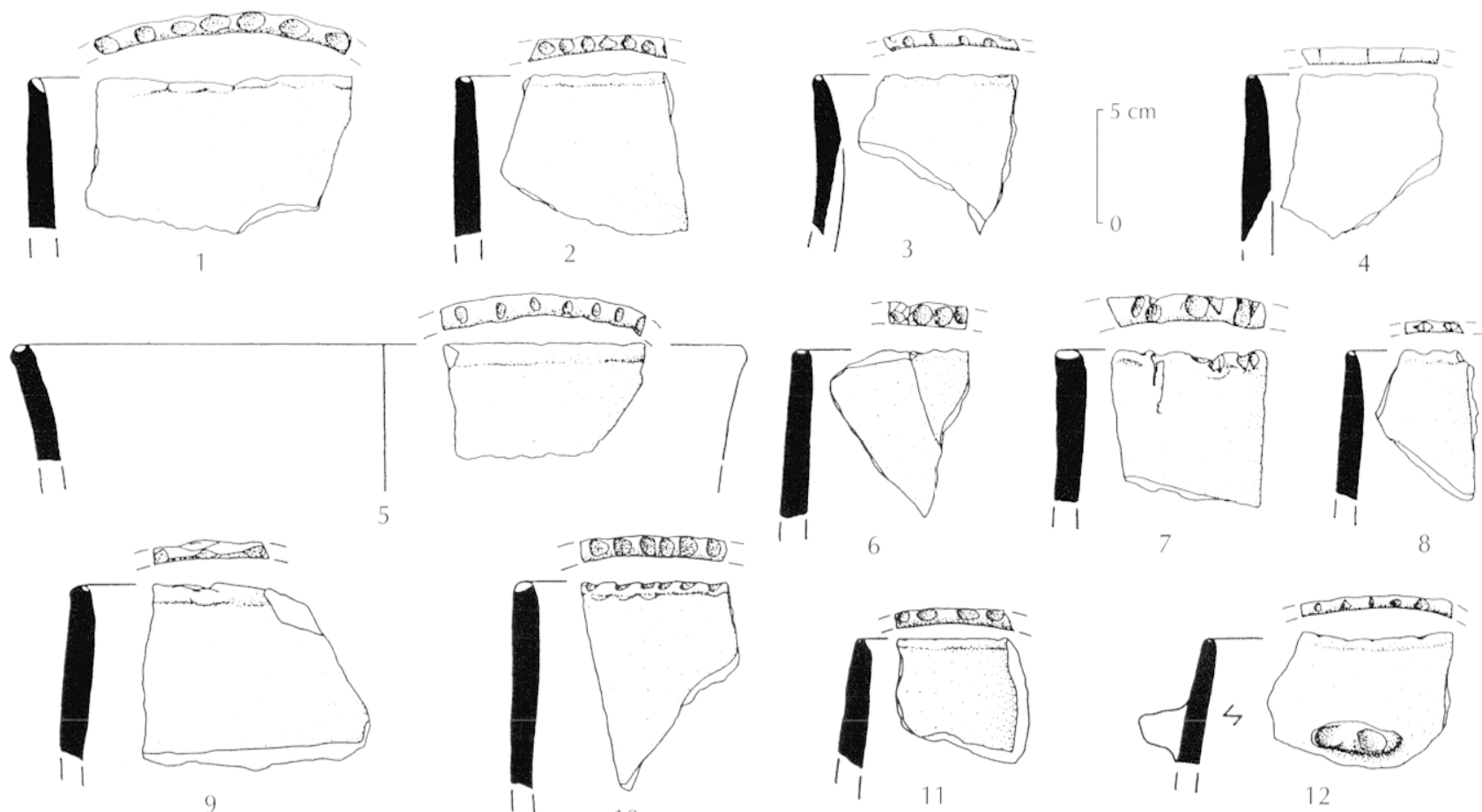

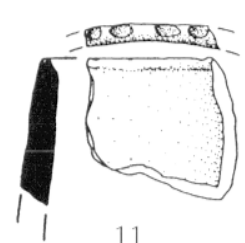

11

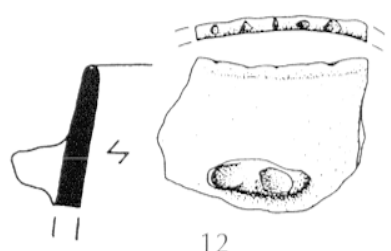

12
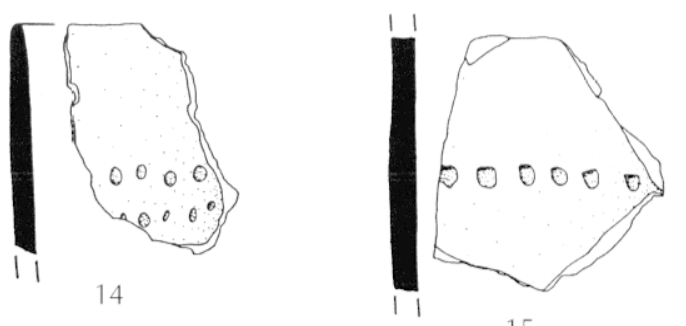

15
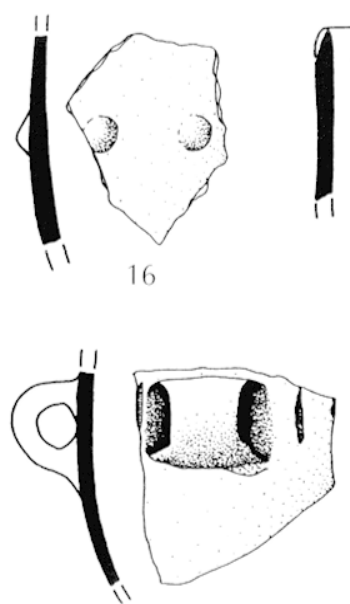

19
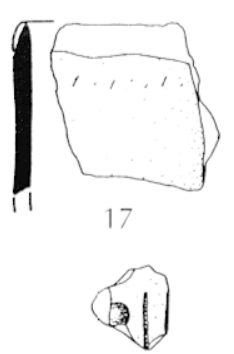

20

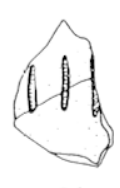

21
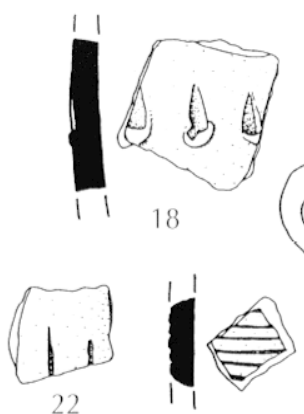

24

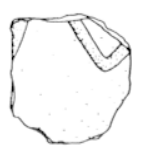

(II)

25

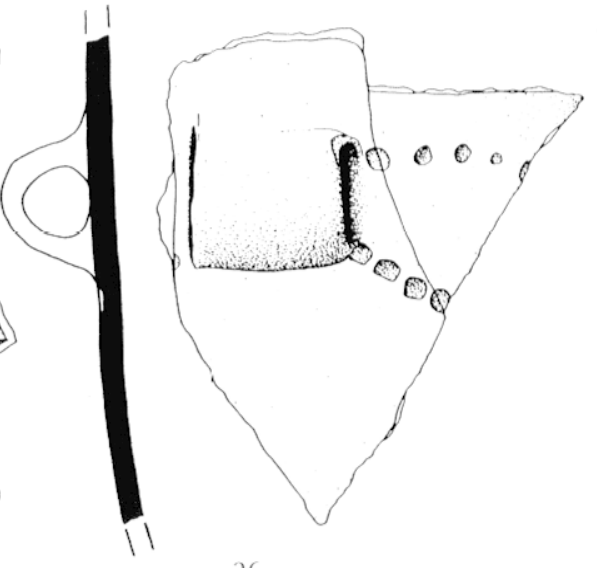

26

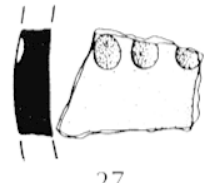

27

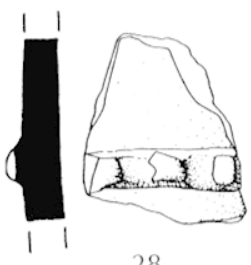

28

23

Fig. 22 - Céramique du Néolithique final, décors (fosses, couches 2a et 2b) : 1-13, impressions sur lìre; ;4, 15, 18, 26, 27, impressions sur panse ; 16, pastilles appliquées ; 17, 19-22, 24, 25, incisions; 23, cannelures en " $V$ ";28, cordon impressionné. 


\section{LES DATATIONS ABSOLUES}

Trois datations radiocarbone ont été effectuées sur des charbons en petite quantitć provenant du comblement de fosses au mobilier abondant. La position de ces échantillons n'ayant pas été relevée lors de la fouille, nous ignorons leur degré de fiabilité.

- Fs 3 : (Ly-8945) $3875 \pm 55$ BP ; 2457 (2390, 2389, 2333)

à 2207 avant J.C.C.

- Fs 4 : (Ly-8946) $3835 \pm 55$ BP ; 2397 (2283) 2148 avant J.-C.

- Fs 5 : (Ly-8947) 4200 $\pm 50 \mathrm{BP}$; $2884(2873,2798,2780$, 2711, 2709) 2669 avant J.-C.

Calibration à 1 sigma d'après Stuiver et Pearson (1993).

Par rapport à la chronologie languedocienne (Gasco, 1991), les deux premières datations se situent dans une plage de temps correspondant à l'extrême fin du Chalcolithique et au début de l'Âge du Bronze, tandis que la dernière indique une phase récente du Néolithique final ou ancienne du Chalcolithique. La bipolarité de ces résultats n'a pas été perçue lors de l'étude du mobilier qui semblait parfaitement homogène. Cette disparité, dans un contexte que l'on a quelques difficultés à délimiter culturellement, accroît lcs problc̀mes plutôt qu'elle ne les simplifie. Le créneau chronologique proposé par les deux premières mesures se révèle difficile à retenir à l'examen du mobilier, de la céramique surtout. En effet, l'absence de tout profil segmenté sur plus de 3000 tessons reste pour nous le meilleur indicateur chronoculturel, auquel on peut ajouter l'inexistence de décors typiques du Fontbouisse, pourtant bien présents par ailleurs dans la vallée de la Tave. Cependant, on notera que des caractéristiques assez proches se retrouvent dans l'un de nos ensembles de comparaison - l'abri de La Vessignée à Saint-Marceld'Ardèche - dont la datation est située entre 2460 et 2281 avant J.-C. ${ }^{3}$.

La datation de la fosse 5 de La Roquette nous paraît plus en adéquation avec les observations typologiques. Toutefois, l'ambivalence de ces résultats nous incite à ne pas en tenir compte dans les hypothèses d'attribution chronoculturelle.

3. ARC $1438: 3882 \pm 50 \mathrm{BP}$, soit $2458-2281$ arant J.C. (calibration à 1 sigma daprès Stuiver, Pearson. 1993).

\section{LA ROQUETTE ET LE NÉOLITHIQUE FINAL BAS RHODANIEN}

\section{LE MATÉRIEL NON CÉRAMIQUE}

Les industries lithiques et osseuses, ainsi que la parure, sont compatibles avec celles du Ferrières et du Fontbouisse.

L'étude des industries lithiques du Néolithique final/Chalcolithique en Languedoc oriental étant pour l'instant peu affinée, excepté pour quelques types d'outils particuliers, les remarques qui suivent sont donc d'ordre général. La bonne représentation des lamelles se retrouve dans certains ensembles ardéchois comme celui de la grotte de Peyroche II à Auriolles (Roudil, Saumade, 1968), la grotte des Huguenots à Vallon-Pont-d'Arc (Roudil, 1965) ou la grotte de Saint-Marcel-d'Ardèche (Gilles, 1988) en contexte ferrérien. Ces éléments sont, à La Roquette, plus frustes que dans les séries chasséennes et la présence de rares lamelles en silex "blond " est peut-être due à une remobilisation. Pour le reste, il s'agit d'un outillage n'ayant mobilisé que peu d'énergie et peu de moyens, si l'on en juge par la généralisation de l'adoption de matériaux locaux, par le nombre de pièces corticales ou subcorticales et enfin par la présence d'artéfacts inclassables typologiquement, peut-être dans certains cas des chutes réutilisées.

L'industrie osseuse est bien caractérisée. La présence marquée du bœuf, des pointes entières et, surtout, la prédominance des pièces tirées d'esquilles évoquant un outillage à l'économie s'accordent avec ce qui a été décrit pour le Néolithique final languedocien et provençal (Sénépart, 1995).

Enfin la parure est constituée d'objets qui n'ont que peu de valeur chronoculturelle.

\section{LA CÉRAMIQUE}

Les formes céramiques de La Roquette s'intègrent pleinement au Néolithique final de type Ferrières (fig. 23). Certes, nombre d'entre elles n'ont pas de réelle valeur chronoculturelle, mais certains éléments peuvent étayer cette impression. L'absence totale de formes carénées nous semble éloquente, non seulement d'un point de vue chronologique, mais également par comparaison avec le Néolithique final de type Treilles, Vérazien et 

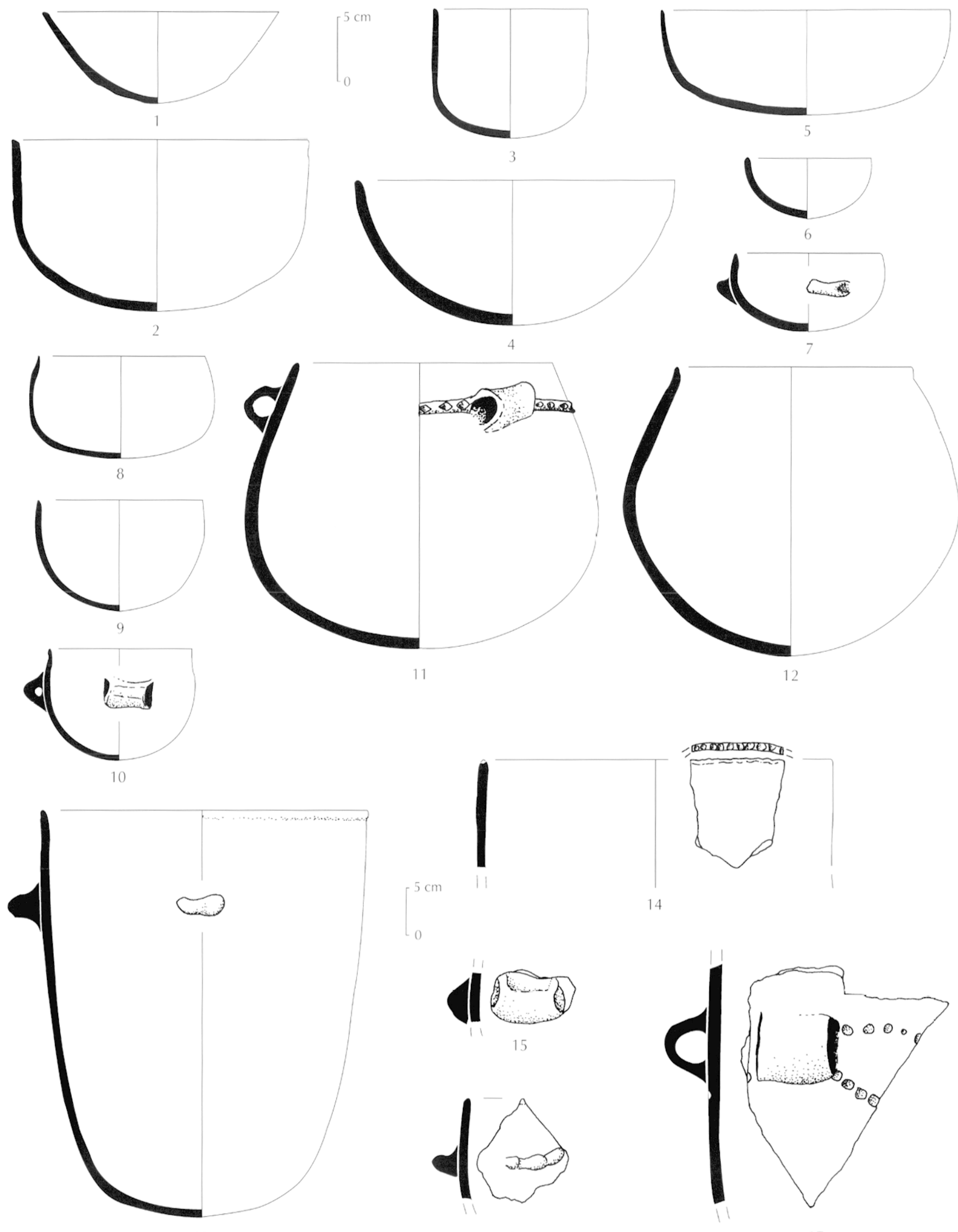

Fig. 23 - Synthèse des formes, des éléments de préhension et des décors céramiques du Néolithique final de La Roquette. 
Saint-Ponien, où ces éléments, quoique en nombre parfois restreint, sont bien mieux représentés que dans le Ferrières ${ }^{4}$. En même temps, il semble que les formes basses à base de demi-ellipsoïde horizontale soicul plus fréquentes dans la céramique du Néolithique final languedocien que dans celle de la Provence. Dans cette dernière région, en effet, le Couronnien semble bien moins concerné par ce type de forme; il en résulte un corpus encore plus paurre, excepté pour quelques types rares comme les tians ou les vases globuleux à col rétréci. Cetto remarque n'est pas pleinement valable pour des groupes tels que le Fraischamp et le Rhône-Ouvèze où l'influence languedocienne est manifeste au niveau des décors de certaines formes (Muller, D’Anna, 1986 ; Sauzade et al., 1990). De même, les lères infléchies (contre-courbes) présentes sur certains rases de petites et movennes dimensions à La Roquette et dans la plupart des ensembles ferrériens, quoique toujours peu nombreuses, sont quasiment absentes dans le Couronnien. Là encore, les groupes du Fraischamp et de Rhône-Ouvèze se distinguent.

C'est essentiellement l'indigence des décors qui distingue La Roquette du Ferrières. Dans cette culture, selon les ensembles, le taux de décors peut certes varier mais, d'après les données que nous possédons, il ne descend que très rarement au-dessous de $20 \%$ pour les séries suffisamment représentatives. De plus, les décors représentés à La Roquette sont rarement les plus typiques du Ferrières. La technique de l'impression est de loin la plus utilisée et, malgré les variations constatées entre les différents faciès géographiques du Ferrières "pur " ${ }^{5}$ sur le plan des techniques décoratives (Guther», 1984), aucune région ne privilégic l'impression. Cette technique reste au second plan, marquée par une extension chronologique qui dépasse le cadre du Ferrières puisqu'on la retrouve avec des thèmes identiques dans le Fontbouisse.

Trois séries de comparaison sont possibles à partir de cette caractérisation : tout d'abord, avec les ensembles

\footnotetext{
4. I a seule série ferrérienne qui, à notre comnaissance. possède des formes carénées est celle de la grotte des Pins à Blandas, (rard (Guther\%, 1984). La position géographique de ce gisement, en bordure des Causses. incite à évoquer de possibles contacts arec le groupe des Treilles pour expliquer cette presence.

5. C̈est-à-dire sans tenir compte des faciès périphériques du Ferrières (Guther\% 1984: Guther\%, Jallot, 1995).
}

de transition entre le (hasséen et le Néolithique final où les décors ferrériens sont encore très discrets; ensuite, avec quelques éléments attribués à la transition Ferrières/Fontbouisse; enfin, arec certains giscments situés en marge du Ferrières qui sont également peu décorés.

\section{Les ensembles de TRANSition enTRE le Chasséen ET LE NÉOLITHIQUE FINAL MÉRIDIONAL}

Cette période a été essentiellement reconnue dans un secteur géographique comprenant le Languedoc occidental, les Causses, le littoral et l'arrière-pays montpelliérain. Ce qui rapproche les ensembles tels que l'Avencas à Brissac, Hérault (Gutherz, Coste, 1974; Gutherz, 1984), la couche 4 b de La Boucle à Corconne, Gard (Coularou et al., 1980; Gutherz, 1984) ou la couche 7 de FontJurénal à Conques-sur-Orbiel, Aude (Guilaine ot al., 1990), du (Chasséen récent de Sargel à Saint-Rome-deCernon, Avevron (Costantini, 1984), c'est un polymorphisme dont le couple carènes/cordons semble être la constante. La série de l'Avencas, datée entre 3616 et 3339 avant J.-C. ", possède plusieurs vases dont la finition brillante est proche de certaines coupes de La Roquette. Mais cette caractéristique n'est pas totalement absente au Ferrières (au Serre de Bernon à Laudun dans le Gard, notamment), et elle est courante au Fontbouisse où elle s'applique souvent à des récipients dont l'extrême finesse de la paroi évoque certains des beaux vases de notre corpus (fig. 14, n"s 7,9 et fig. 15, $\mathrm{n}^{\circ} 1$ ).

Dans le Languedoc rhodanien et en Provence, cette période, mal connue jusqu'alors, a bénéficié des interventions conduites sur le tracé du TGV. Plusieurs sites sont à inclure dans une plage chronologique située entre 3700 et 2900 avant J.-C. ${ }^{7}$, notamment La Grange des

6. MC $1253: 4650 \pm 100 \mathrm{BP}$ (calibration à 1 sigma d'apres Suiver, Pearson, 1993).

7. Grange des Merveilles, foyer 1 : ARC $1393: 4777 \pm 50 \mathrm{BP}$, soit 36393388 avant J.-C. La liaison stratigraphique entre ce forer et le niveau ayant livé le mobilier est incertaine. Nécropole de Château-Blanc, tertre 1 : KTH 15735: $4155 \pm 75 \mathrm{BP}$, soit $2880-2586$ avant J.-C. et ETH $15260: 4385 \pm 60$ BP, soit $3090-2914$ avant J-C. ; tertre IVa : F.TH 15732 : $4390 \pm 85 \mathrm{BP}$, soit $3257-2909$ atant J-C.; ; tertre V': E.CH $15621: 4620 \pm$ 60 BP, soit 3499-3342 arant J.-C. I.es Ribauds, structure 11 : At 24901 : $4775 \pm 55$ BP, soit 3639-3387 avant J.-C. Le Duc, couche 8 : AA24900 : $4770 \pm 85 \mathrm{BP}$. soit $3646-3380$ arant J.-C. (calibration a 1 sigma d'après Stuiver, Pearson, 1993). 
Merveilles à Rochefort-du-Gard, Gard (Monnet et al., 1997), Les Juilléras, Le Duc et Les Ribauds à Mondragon, Vaucluse (Lemercier et al., 1997, 1998; Margarit, Renault à paraître; Margarit et al., à paraîtrc) ct Château-Blanc à Ventabren, Bouches-du-Rhône (Hasler et al., 1996). Si Les Ribauds et La Grange des Merveilles ont un lien avec la tradition chasséenne, il semble que les autres s'en dégagent. Les carènes sont chaque fois présentes et sont associées à des formes simples ayant des affinités avec le Néolithique final.

Les comparaisons avec les ensembles présumés de transition Chasséen/Néolithique final restent donc très superficielles et l'absence de carènes à La Roquette nous semble un argument typologique de poids contre une attribution à cette période.

\section{LES ÉLÉMENTS ATTRIBUÉS À LA TRANSITION ENTRE FERRIÈrES ET FONTBOUISSE}

Cette transition semble difficile à concevoir. Il y a plusieurs raisons à cela. D'abord, la continuité entre les deux cultures apparaît clairement (jacobieski, 1988 ; Gutherz, Jallot, 1995) et semble plus prononcée encore, nous semble-t-il, qu'entre le Chasséen et le Ferrières. Les formes simples ferrériennes se retrouvent dans le Fontbouisse, de même que certains thèmes clécoratifs, de sorte que l'on observe dans l'ensemble un enrichisscment du corpus plutôt qu'un renouvellement, durant la deuxième période. Ensuite, le morcellement en faciès géographiques - dont les limites sont d'ailleurs très proches pour les deux périodes - ne facilite pas la généralisation des observations locales sur l'évolution typologique. Enfin, le recouvrement partiel des plages chronologiques dévolues aux deux cultures (Gasco, 1991) constitue un obstacle à la recherche d'une telle transition.

Les ensembles proposés par X. Gutherz et L. Jallot (1995) pour illuster cette période sont les suivants : Forton à Lansargues et Plaine de Chrétien à Montpellier dans l'Hérault, la fosse 663 de Moulin-Villard à Caissargues, Campguivard à Collias, le niveau intermédiaire de la grotte des Meules à Rochegude et Les Bruyères à Saint-Julien-de-Peyrolas dans le Gard. L'originalité de ces ensembles n'a pas encore été clairement définic. Ces derniers semblent marqués par la présence de profils sinueux et celle, discrète, de formes plus ou moins segmentées, ainsi que par la relative rareté des décors parmi lesquels les thèmes de prédilection du Ferrières sont sous-représentés, voire absents dans le cas des Bruyères et de Moulin-Villard. La sous-représentation des décors typiques, observée sur d'autres sites comme celui du Bois de Mintau à Calvisson dans le Gard (Roger, Valette, 1983), se fonde sur des comparaisons avec les trop rares décomptes, essentiellement issus de la thèse de X. Gutherz (1984). Notons également que les gisements de Plaine de Chrétien et Forton se situent dans une zone géographique où les décors sont habituellement peu présents au Ferrières comme au Fontbouisse (faciès littoral des deux cultures).

La Roquette montre des affinités avec Les Bruyères et Moulin-Villard, malgré quelques carènes. Les arguments qui permettent ces rapprochements ne se limitent pas à la nature des décors et à leur indigence. On trouve dans ces trois ensembles quelques éléments ayant un rapport avec le domaine provençal, et dont la présence ne peut être expliquée par l'hypothèse chronologique que nous venons de développer. Dans le cas des Bruyères, la proposition d'un faciès rhodanien, plusieurs fois mentionnée dans les différentes publications, rend compte de ces affinités avec le Néolithique final d'outre-Rhône.

\section{LES FACIÈS RHODANIENS PÉRIPHÉRIQUES}

La notion de "faciès rhodanien apparenté au Ferrières" (Gutherı, Jallot, 1995, p. 240) a été employée pour désigner l'ensemble du Mas de Miraman à Nîmes (Roger, Guthery, 1986). Les formes céramiques de cette station s'intègrent bien dans le corpus ferrérien, mais les décors à base de cordons et d'impressions sont assez particuliers. On retrouve la prédominance des impressions parmi les rares décors présents au Jas des Chèvres à Allan dans la Drome (Beeching, 1980). Dans cette station, ce sont surtout les lères qui, comme à La Roquette, portent ce type de décor:

Les ensembles peu décorés semblent, pour l'instant, micux attestés dans un horizon chronologique contemporain du Fontbouisse, mais certains peuvent réunir plusicurs occupations difficiles à démêler. Ils se situent, pour l'essentiel, dans la basse et moyenne vallée de l'Ardèche : Les Bruyères à Saint-Julien-de-Peyrolas, Gard (Gilles, 1975), l'abri de La Vessignée à Saint-Marcel-d'Ardèche, Ardèche (Beaume, 1996), Le Puits du Fakir à Balazuc, Ardèche (Mouyon et al., à paraître), Le Serre de Boidon et Le Ranc d'Aven à Grospierres, Ardèche (Gros, 1971, 
1974) auxquels il nous semble opportun d'adjoindre la fosse 663 de Moulin-Villard à Caissargues, Gard (Échallier, Jallot, 1992). Ces ensembles ont en commun l'adoption de formes à profil segmenté issucs du Fontbouissc. Les décors sont essentiellement représentés par des pastilles appliquées et des impressions sur la lère et la panse. Quelques formes originales sont constituées par des jarres globuleuses et profondes ou étroites et profondes à ouverture rétrécie.

L'indigence des décors est attribuée à la proximité de cultures à céramique peu ornée présentes en rive gauche du Rhône. En effet, les influx possibles diffusés par une culture comme le Couronnien, où les formes céramiques sont très peu caractérisées et où les décors sont quasi absents, sont plus difficiles à percevoir que ccux d'unc culture intégrant des décors céramiques bien typés comme le Ferrières qui a largement diffusé du côté provençal : " couronno-Ferrières " et "tardi-Ferrières " (Montjardin, 1970, 1984), Fraischamp et Rhône-Ouvèze (Muller, D’Anna, 1986 ; Sauzade el al., 1990 ; D’Anna, 1995a), et en direction du nord-est : Néolithique final des lacs alpins et jurassiens, jusqu'en Suisse occidentale (Strahm, Thévenot, 1976 ; Pétrequin et al., 1988; Wolf, 1991 ; (Giligny et al., 1995).

Plusieurs éléments couronniens sont cependant assez spécifiques. Il s'agit, pour lcs formcs cćramiques, de vases très pansus à ouverture rétrécie et de quelques types spécifiques tels que les bassins ou tians, et pour les décors, de cordons courts en pendentif ou en faisceau partant des éléments de préhension et de cordons fins en portée longue simple, ondée ou en cherrons. Les éléments de préhension, peu variés dans le gisement éponyme, et dans la basse Provence en général, le sont bien davantage dans les sites plus septentrionaux comme Les Lauzières à Lourmarin, La Brémonde à Buoux dans le Vaucluse (D'Anna et al., 1989), La Citadelle à Vauvenargucs dans les Bouches-du-Rhône (D'Anna, 1989), où ils semblent véhiculer une charge identitaire assez importante, à l'image d'autres cultures où les décors sont rares. Les types les plus spécifiques sont les mamelons prismatiques, les languettes prolongées en cordon, les bobines, les prises semi-cylindriques pseudofuniculaires, les prises en « $H$ » variées. Les éléments de préhension et les décors semblent éroluer pendant toute la séquence couronnienne ; les premiers s'assimilant peu à peu aux seconds. L'ornementation de la céramique reste secondaire dans cette culture; il n'y a jamais de notion de registre décoratif comme c'est le cas dans le Ferrières, le Fontbouisse, le Fraischamp et le RhôneOuvèze, et on ne trouve pas, dans la phase récente du Couronnien, l'association étroite entre les formes céramiques, les éléments de préhension et les décors qui caractérise asse $z$ bien le Fontbouisse. L'industrie lithique couronnienne se distingue par la présence de lames étroites et épaisses à retouche marginale ou foliacée et d'armatures foliacées de forme amygdaloide, pistilliforme ou allongée.

Comme nous l'avons souligné, outre l'absence de cordons qui reste un argument négatif, certains éléments de préhension de Ia Roquette trouvent des comparaisons dans la sphère provençale. Il s'agit des prises pseudofuniculaires et en "pseudo-II ", des mamclons prismatiques et des bobines. Aux Bruyères, une prise tunnelliforme associée à deux cordons obliques courts évoque le Couronnien. Une anse en bobine est présente à MoulinVillard. À Allan, certains éléments comme les coupes en calotte profondes, les lames étroites et épaisses très retouchées, les pointes de type pistilliforme, amygdaloïdes ou allongées semblent redevables à l'aire provençale (Beeching, 1980). Enfin, aux Bruyères, à La Vessignée, au Puits du Fakir et au Ranc d'Aven, plusieurs jarres pansues à ouverture rétrécie peuvent dériver de certaines formes couronniennes (Beeching, 1980 ; Gutherz, 1984).

André D'Anna (1995b) a proposé d'adjoindre à ce contexte culturel les ensembles provençaux sépulcraux du nord du Vaucluse. Ce sont essentiellement la couche 5 de l'hypogée des Crottes à Roaix et la couche 2 de l'hypogée du Capitaine à Grillon (Sauzade, 1983). Les formes céramiques sont très simples et très rarement décorées.

Il est difficile de ne pas évoquer la question des contacts avec la moyenne vallée du Rhône, en retour de ceux identifiés dans les stations des lacs alpins et jurassiens, pour appréhender ces ensembles. Là encore, le manque de caractère du Néolithique final dans ces régions fait obstacle aux comparaisons. Toutefois, en Provence, la forme générale des récipients des couches 4 et 5 de Roaix (jarres profondes à paroi droite ou légèrement tronconique, gobelets tulipiformes) et des éléments de préhension (fréquents boutons et mamelons coniques, parfois disposés par quatre près du bord) peut évoquer l'environnement culturel de la moyenne vallée du Rhône et de Suisse occidentale (Lücherz, groupes de Clairvaux et de (halain). On retrouve des éléments de 
préhension semblables sur le site de La Clairière (Fraischamp).

S'il est difficile de comparer chronologiquement tous les sitcs appartcnant aux faciès bas rhodaniens, il reste que sur le plan culturel un même contexte pourrait les unir. La position de La Roquette non loin du Rhône est un argument en faveur de cette hypothèse culturelle. Il n'est pas impossible que les contacts avec la rive gauche du Rhône aient des antécédents au début du Néolithique final. Les thèmes décoratifs tels que les impressions, particulièrement récurrentes, et l'extrême rareté des cordons simples permettent des rapprochements convaincants avec La Roquette.

$$
*
$$

Les groupes de Ferrières et de Fontbouisse sont sans doute les mieux définies pour le Néolithique final méridional, en particulier grâce à leurs styles céramiques décorés. Bien qu'elles soient environnées de cultures où l'ornement est rare, elles conservent cette caractéristique d'un bout à l'autre de leur aire d'extension et les faciès définis par Xavier Gutherz (1984) restent indiscutables. Cependant il existe quelques ensembles représentant des "faciès "exotiques" " (Jallot, 1988, p. 82), soit parce que leur structure décorative ne correspond pas à un faciès déjà défini, comme la station du Bois de Mintau à Calvisson, Gard (Roger, Valette, 1983) ou celle de Campguivard à Collias, Gard (Jallot, 1988), soit parce que leurs éléments décoratifs sont déficitaires. La valeur chronologique ou culturelle de ces divergences est difficile à démontrer. Seuls des décomptes systématiques sur les ensembles ferrériens permettraient d'évaluer l'importance, et, peut-être, d'appréhender la nature de ce type d'observations qui, pour l'instant, entretient une certaine confusion entre ce qui touche au domaine des contacts culturels et ce qui renvoie à une problématique chronologique (reconnaissance de "transitions ", entre autres), comme le montre le cas des Bruyères.

Nous avons effleuré ici le problème des contacts périrhodaniens au Néolithique final-Chalcolithique. Les relations entre le Languedoc, la Provence et la moyenne vallée du Rhône sont essentiellement attestées par la diffusion des cultures du Languedoc oriental qui semblent être, dans le Jura, mais surtout en Provence, une composante importante de la genèse de différents styles ou groupes : style de Clairvaux, groupes du Fraischamp et de Rhône-Ouvèze. Certains sites, comme ceux d'Avignon, présentent une situation complexe, puisqu'il semble possible d'y reconnaître, en plus des étapes se rattachant au Fraischamp et au Rhône-Ouvèze, des éléments du Ferrières et du Fontbouisse vrais (D'Anna, 1995a et b). Cela s'applique également à Escanin où il faut ajouter le Couronnien (id. ibid.). En rive droite du Rhône, ces contacts sont plus difficiles à percevoir. Ils seraient matérialisés par quelques indices : la rareté des décors où les incisions, les cannelures et les cordons sont moins présents que certains motifs généralement secondaires dans le Ferrières et le Fontbouisse (impressions, cordons impressionnés, pastilles) et la présence de bobines, de prises pseudo-funiculaires, en " $\mathrm{H}$ ", ou prolongées par des cordons verticaux ou obliques courts. Pour avoir une signification culturelle, plusieurs indices doivent être associés dans un même ensemble. À Allan et dans d'autres ensembles drômois (Beeching, 1980), les industries lithiques montrent une double influence, provençale et languedocienne.

Il reste à présent à mieux cerner la nature de ces relations qui pourraient s'éclaircir s'il était possible de reprendre l'étude des ensembles provençaux et drômois dans cette optique. 


\section{ANNEXE}

\section{ÉTUDE ARCHÉOZOOLOGIQUE DE LA FAUNE DU NÉOLITHIQLE FINAL}

\author{
Vianney FoRkSI
}

L'étude archéozoologique du site de La Roquette a été menée rapidement dans le but d'obtenir un aperçu de la composition du mobilier faunique sur lequel puisse s'appuyer une comparaison avec le lot d'outils issus de l'industrie osseuse ${ }^{8}$. L'interprétation archéozoologique débouchant sur l'alimentation et l'élevage ne pouvait $a$ priori être menée précisément, mais la qualité générale des données de fouilles et du lot osseux et dentaire nous permet de considérer que la majeure partie de l'information a été récupérée au cours de cette étude simplifiée.

Le matériel provient de huit fosses (Fs 3 à Fs 10), de trois sondages (S4, S5 et S7) et d'un ensemble de couches regroupées sous le vocable de " décapage " (décap.), le tout attribué au Néolithique final. Lors de la fouille, le ramassage des restes s'est fait manuellement le plus exhaustivement possible, mais aucun tamisage n'a été effectué.

\section{MÉTHOdeS D'ÉTUDE}

L'identification spécifique des ossements s'appuie sur des ouvrages généraux d'ostéologie animale (Schmid, 1972 ; Barone, 1976), sur notre collection personnelle de comparaison et sur des travaux plus précis que nous mentionnerons lors de leurs utilisations.

Les comptages des ossements sont effectués en nombre de restes (NR).

L'analyse des âges des animaux repose sur :

- trois grandes classes, infantile, juvénile et adulte, fondées sur la taille et l'aspect des ossements, et quantifiées par le nombre minimum d'individus (NMI) de comparaison ;

- les éruptions et usures dentaires des mâchoires (d'après Silver, 1969) ;

- la plupart des soudures épiphysaires osseuses (d'après Curgy, 1965).

8. Nous remercions, pour leur aide, J. Peters de l'Institut für Palacoanatomic, Domestikationsforschung und Geschichte der Tiermedexin der Ciniversität München et I. Carrere du Centre d'Anthropologic, EHF.SS de Toulouse.

\section{RÉSULTATS}

Le tableau I présente les nombres de restes, le tableau II les nombres minimums d'individus, et le tableau III, les nombres de restes par organe pour les taxons les plus abondants.

À l'exception des pièces d'un squelette de chien en connexion lors de la fouille, 1656 restes ont été dénombrés dont seulement 486 ont pu être déterminés, soit $29,3 \%$.

\section{État de la matière osseuse}

La matière osseuse est bien conservée. L'action physico-chimique du sol est faible. De plus, les surfaces osseuses ne sont pas altérées, notamment par les radicelles végétales.

\section{Les espèces}

\section{Les ovicaprins}

Tous les organes squelettiques sont présents (tabl. III). En valeurs absolues, les restes de la tête, des principaux os longs des membres, humérus, radius, fémur, tibia et métatarse, et surtout des côtes, sont abondants. Cependant les côtes - et à un degré moindre la tête - sont beaucoup moins fréquentes, si leur quantité est rapportée à la composition squelettique théorique. Ainsi, 14 humérus correspondent à $14 \times 13$ = 182 côtes, soit quatre fois plus que ce qui a été identifié. Ainsi ce sont principalement les os longs des membres qui sont le plus conservés par rapport aux squelettes initiaux. Le rachis - dont un sacrum et trois vertèbres lombaires probablement en connexion sont présents dans les décapages - et les phalanges sont peu fréquents.

Les ovins sont majoritaires en nombre de restes mais les caprins sont bien présents avec $37 \%$ des restes (Boessneck, 1969 ; Payne, 1985) (tabl. IV). Un peu moins du tiers des animaux sont morts à l'âge adulte (tabl. II), les juvéniles étant presque deux fois plus nombreux que les infantiles. Les âges dentaires montrent que les jeunes adultes, entre 2 et 4 ans, sont absents (tabl. V), mais certaines épiphysations osseuses (fémur proximal, humérus proximal) plaident pour leur présence (tabl. VI), à moins 
Tabl. I - Nombre de restes délerminés par structure : $F$, fosse; $S$, sondage;

* les éléments d'un squelette entier de chien n'ont pas été intégrés dans ce décompte.

\begin{tabular}{|c|c|c|c|c|c|c|c|c|c|c|c|c|c|c|}
\hline & Fs3 & Fs4 & Fs5 & Fs6 & Fs7 & Fs8 & $\mathrm{Fs}^{*}$ & Fs10 & décap. & 54 & S5 & 57 & Total & $\%$ \\
\hline Ovicaprins & 7 & 24 & 29 & 31 & 28 & 3 & 11 & 5 & 22 & 4 & 38 & 19 & 221 & 56,8 \\
\hline Porcins & & 4 & 1 & & & & & & & & 3 & & 8 & 2,1 \\
\hline Bovins & 2 & 9 & 16 & 5 & 7 & 1 & 6 & & 47 & 3 & 32 & 8 & 136 & 35,0 \\
\hline Chien & 1 & & & & & & 5 & 1 & 2 & & & & 9 & 2,3 \\
\hline Lapin & & & & & & & & & 2 & & 1 & & 3 & 0,8 \\
\hline Micromammifères & & & & & & & 1 & & & & & & 1 & 0,3 \\
\hline Batraciens & 8 & & 1 & & & & 1 & & 1 & & & & 11 & 2,8 \\
\hline Total NR & 18 & 37 & 47 & 36 & 35 & 4 & 24 & 6 & 74 & 7 & 74 & 27 & 389 & 23,5 \\
\hline Verlèbres & & 1 & 1 & 2 & & & 3 & & 11 & & 2 & 2 & 22 & 1,3 \\
\hline Ovicaprins & & & 1 & 2 & & & 1 & & 5 & & 2 & 1 & 12 & \\
\hline Bovins & & 1 & & & & & 2 & & 6 & & & 1 & 10 & \\
\hline Côtes & 2 & 6 & 3 & 13 & 9 & & 2 & & 18 & 1 & 16 & 5 & 75 & 4,5 \\
\hline Ovicaprins & 1 & 4 & 2 & 10 & 7 & & & & 11 & & 6 & 4 & 45 & \\
\hline Porcins & & & & & & & 1 & & & & & & 1 & \\
\hline Bovins & 1 & 2 & 1 & 3 & 2 & & 1 & & 7 & 1 & 10 & 1 & 29 & \\
\hline Inctélermincés & 25 & 42 & 249 & 117 & 231 & 7 & 43 & 9 & 199 & 36 & 1.38 & 74 & 1170 & 70,7 \\
\hline Total restes & 45 & 86 & 300 & 168 & 275 & 11 & 72 & 15 & 302 & 44 & 230 & 108 & 1656 & 100 \\
\hline
\end{tabular}

Tabl. II - Nombre minimum d'individus par structure et parts globales de viande par taxon (Columeau, 1991) : Fs, fosse; $S$, sondage.

\begin{tabular}{|c|c|c|c|c|c|c|c|c|c|c|c|c|c|c|c|c|}
\hline & & 58 & Hs & Fs 4 & Fs3 & Fs6 & Fs? & Fs & Fs10 & decap. & S4 & S5 & S7 & Total & $\%$ & $\%$ viande \\
\hline Ovicaprins & infantiles & & 1 & 1 & & 1 & & & 1 & 1 & & 1 & 1 & 7 & 13,7 & \multirow[t]{3}{*}{13,4} \\
\hline & juvéniles & 1 & 2 & 1 & 1 & 1 & 1 & & 1 & 2 & & 1 & 1 & 12 & 23,5 & \\
\hline & adultes & 2 & 5 & 2 & 1 & 2 & 2 & 2 & 1 & 9 & 2 & 2 & 2 & 32 & 62,7 & \\
\hline Porcins & infantiles & 1 & & 1 & & & & & & & & & & 2 & & \multirow[t]{2}{*}{3,2} \\
\hline & adultes & & 1 & I & & & & & & & & 1 & & 3 & & \\
\hline Bovins & infantiles & & 1 & & & 1 & & & & & & & & 2 & 7,1 & \multirow[t]{3}{*}{83,5} \\
\hline & juvéniles & 1 & & & & & 1 & & & & & 1 & 1 & 4 & 14,3 & \\
\hline & adultes & 1 & 2 & 1 & 1 & 1 & 1 & 1 & 1 & 9 & 1 & 2 & 1 & 22 & 78,6 & \\
\hline
\end{tabular}

qu'il ne s'agisse d'animaux dont une castration a retardé les épiphysations. Un os coxal (Fs 4) est conformé comme celui d'une femelle pluripare.
Morphologiquement, les cornillons de chèvre sont droits mais en se recourbant progressivement vers l'arrière. Une diaphyse de métacarpe montre une morpho- 
Tabl. III - Nombre de restes par organe: *, squelette entier exclu.

\begin{tabular}{|c|c|c|c|c|}
\hline & Ovicaprins & Bovins & Porcins & Chien* \\
\hline os crâniens & 19 & 32 & & \\
\hline maxillaire & 2 & 4 & & \\
\hline dents supérieures & 12 & 4 & & \\
\hline mandibules & 18 & 11 & 2 & 1 \\
\hline dents iniérieures & 11 & 7 & & \\
\hline scapula & 6 & 2 & 2 & \\
\hline humérus & 14 & 9 & 3 & \\
\hline radius & 19 & 7 & & \\
\hline ulna & 9 & 2 & & 1 \\
\hline carpe & & 5 & & \\
\hline coxal & 7 & 3 & & \\
\hline iémur & 20 & 4 & 1 & \\
\hline tibia & 27 & 6 & 1 & \\
\hline fibula & & & & 1 \\
\hline talus & 3 & 3 & & \\
\hline calcanéus & 4 & 5 & & 1 \\
\hline tarse & 2 & 5 & & \\
\hline métacarpe & 5 & 4 & & \\
\hline métatarse & 17 & 7 & & 1 \\
\hline métapode & 7 & 2 & & 1 \\
\hline phalange 1 & 9 & 5 & & \\
\hline phalange 2 & 1 & 5 & & \\
\hline phalange 3 & 2 & 2 & & \\
\hline divers & & 2 & & \\
\hline atlas & 2 & & & \\
\hline axis & 3 & & & \\
\hline vertèbres cervicales & 2 & & & \\
\hline vertèbres thoraciques & 3 & 8 & & \\
\hline côtes & 45 & 29 & 1 & 2 \\
\hline vertèbres lombaires & 6 & 2 & & 1 \\
\hline sacrum & 2 & & & \\
\hline vertèbres caudales & 1 & & & \\
\hline Total NR & 278 & 175 & 10 & 9 \\
\hline Traces de brûlure & 61 & 12 & 1 & 2 \\
\hline
\end{tabular}

logie proche de celle de métacarpes de mouton trouvés sur deux sites du Languedoc oriental, un du Néolithique et un du Chalcolithique (Forest, 1997) : os long très gracile dont la face crâniale diaphysaire est très cintrée et dont la moitié médiale de la face caudale diaphysaire est pourvue sur toute sa longueur d'une gouttière longitudinale saillante. Cette morphologie très particulière a été rapprochée de celles de moutons ibériques des mêmes périodes par notre confrère J. Peters auquel nous avons montré ces pièces (Driesch, 1972).

Certaines pièces, peu nombreuses mais non comptées, portent des stries et des entailles qui témoignent de désarticulations/désossages de la carcasse et des quartiers. Des impacts de coups sont perceptibles sur les diaphyses d'os longs, mais leur identification n'est pas toujours aisée. Par ailleurs, $22 \%$ des restes d'ovicaprins sont brûlés à des degrés variables, du coup de chaud à la calcination.

\section{Les porcins}

Les quelques restes retrouvés (huit) correspondent à deux infantiles et trois adultes. Une molaire 3 en début d'usure permet d'attribuer un âge de 18-24 mois à un mâle adulte (Fs 4). Les dimensions des ossements sont movennes par rapport à celles connues du porc domestique contemporain.

\section{Les bovins}

Les vestiges proviennent de la plupart des organes squelettiques (tabl. III). Comme pour les ovicaprins, les valeurs absolues de nombre de restes accordent une forte proportion aux côtes, et la comparaison arec un squelette entier fait chuter fortement la fréquence de ces organes. Globalement, aucun organe ne semble véritablement privilégié et il manque les supports osseux du cou, les vertèbres cervicales. Notons que dans une zone de décapage devaient être en connexion premièrement un radius et la rangée proximale des os du carpe, deuxièmement un naviculo-cuboïde, un grand cunéiforme et le métatarse, tous droits, et troisièmement un métatarse, deux phalanges 1 , deux phalanges 2 , deux phalanges 3 , tous gauches. Les deux derniers assemblages appartiennent très vraisemblablement au même animal.

Presque $80 \%$ des 26 individus sont des adultes (tabl. II), mais juvéniles et infantiles sont présents. Les adultes sont jeunes ou matures (tabl. VII et VIII), mais les épiphysations osseuses montrent que les jeunes sont plus nombreux (tabl. VII). Les dimensions des os sont petites à moyennes, similaires aux animaux de cette époque, hauts d'environ $1,10 \mathrm{~m}$ au garrot. Toutefois, il faut noter la présence d'os plus grands, un fragment de métatarse (Fs 4), une extrémité distale d'humérus (décap.), correspondant à des individus voisins de $1,30 \mathrm{~m}$. Régionalement, ces animaux, remarqués dès 1957 par T. Josien, sont régulièrement présents sur les sites du Chalcolithique et du Bronze ancien (Forest, 1997), mais leur statut reste à ce jour indéterminé : petit aurochs, morphotype différent de bœuf domestique, ou forme extrême d'un même morphotype de bovin domestique à variabilité large. 
Tabl. IV - Nombre de restes de mouton et de chèrre par organe el par structure :

Fs, fosse; $S$, sondage; $c$, chèure; ov, mouton.

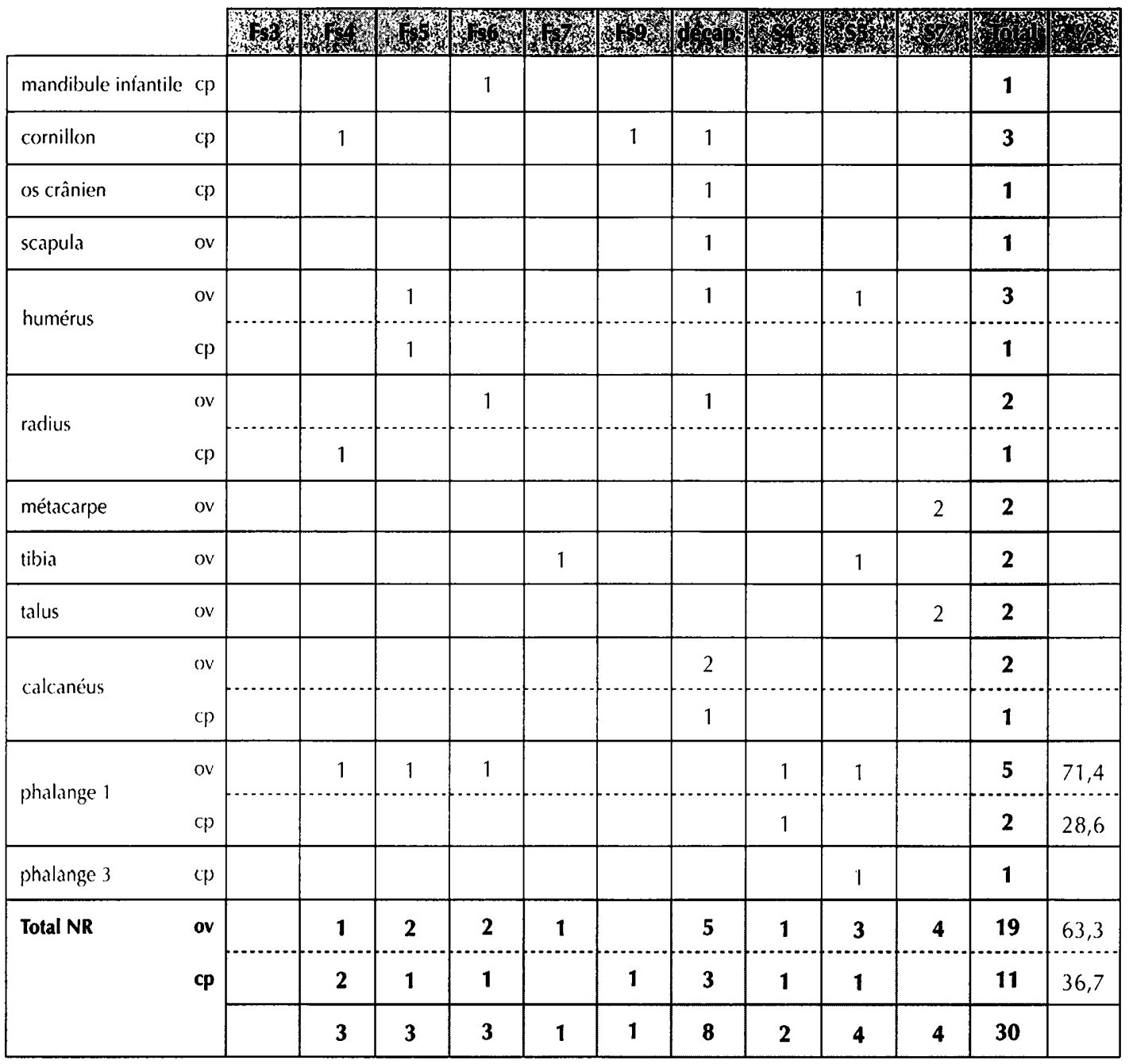

Tabl. V - Morlalité des ovicaprins d'après les mâchoires : Fs, fosse; $S$, sondage; $P$, prémolaire; $M$, molaire ; \pm , en éruption; +, ++, +++, stades d'usure croissante; T, arasée.

\begin{tabular}{|c|c|c|c|c|c|c|c|c|c|c|c|c|c|}
\hline & & & & $\sin x \cos$ & $=0$ & & 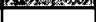 & 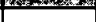 & & 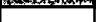 & & & \\
\hline & & $\mathrm{M}_{1+}, \mathrm{M}_{2}-$ & $3<9$ mois & & & & & & & 1 & & 1 & \\
\hline juvénil & & $\mathrm{M} 2+1-$ & \pm 9 mois & & & & 1 & & & & 1 & 2 & \\
\hline & & $\mathrm{M} 2+, \mathrm{M} 3-$ & $9<18$ mois & & 1 & & & & 1 & & & 2 & \\
\hline & & $\mathrm{P} 4++, \mathrm{M} 3++$ & $4<5$ ans & & & & & & 1 & & 1 & 2 & \\
\hline adulte & mature & $\mathrm{P} 4+++, \mathrm{M} 3+++^{+}$ & $6<7$ ans & 1 & & & & 2 & & & & 3 & \\
\hline & vieux & $\mathrm{P} 4 \mathrm{~T}, \mathrm{M3}^{-}$ & $>7$ ans & & & & & 1 & & & & 2 & \\
\hline Total NI & & & & 1 & 1 & & 1 & 3 & 2 & 1 & 2 & 12 & \\
\hline
\end{tabular}


Tabl. VI - Mortalité des ovicaprins adultes d'après les épiphy:sations osseuses : Fs, fosse ; S, sondage.

\begin{tabular}{|c|c|c|c|c|c|c|c|c|c|}
\hline & & \multirow{2}{*}{\multicolumn{2}{|c|}{\begin{tabular}{l|l} 
H54 & Fo
\end{tabular}}} & \multirow[b]{2}{*}{ décap. } & \multirow[b]{2}{*}{ s5 } & \multirow[b]{2}{*}{57} & \multirow[b]{2}{*}{ rotal AR } & \multirow[b]{2}{*}{ rotal } \\
\hline & & & & & & & & & \\
\hline \multirow{5}{*}{ jeune } & épiphysations & tibia distal & & & & & & 1 & \\
\hline & précoces & métacarpe distal & & & & & 1 & 1 & \\
\hline & $\pm 18 \mathrm{~m}$ & métapode distal & 1 & & 1 & & & 2 & \\
\hline & $\pm 2,5$ ans & calcanéus & & & 3 & & & 3 & \\
\hline & $2,5 \leq 3,5$ ans & fémur proximal & 1 & & & & 1 & 3 & \\
\hline \multirow{4}{*}{ mature } & épiphysations & humérus proximal & & & 1 & & & 1 & \\
\hline & tardives & radius distal & & & 1 & & & 1 & \\
\hline & $\pm 3,5$ ans & fémur distal & & & & & 1 & 1 & \\
\hline & & tibia proximal & & 1 & & & & 1 & \\
\hline \multicolumn{3}{|c|}{ Total NR } & 2 & 1 & 6 & & 3 & 14 & \\
\hline
\end{tabular}

Les marques de découpe sont similaires à celles décrites chez les ovicaprins, mais les impacts de coups ou de percussions sont beaucoup plus nets. Le pourcentage d'os brûlés est plutôt faible : $6 \%$.

\section{Le chien}

Les rares vestiges appartiennent à des animaux dc taille moyenne à petite. La fosse Fs 9 contenait le squelette entier d'un animal qui avait autour de 1 an lors de sa mort (épiphysation de l'extrémité proximale de l'humérus en cours).

\section{Les autres taxons}

Les vestiges de lapin, Oryctolagus cuniculus (Callou, 1997), un métatarse, un humérus et un fémur, peuvent aussi bien provenir d'animaux consommés qu'intrusifs.

Cette seconde hypothèse est à retenir pour le micromammifère et les batraciens.

\section{Les indéterminés}

Ils composent $70 \%$ du lot. Une étude plus lente et approfondie aurait pu réduire ce nombre (10\% tout au plus), sans apporter de grandes modifications aux conclusions générales, car ce sont des débris et des esquilles qui témoignent d'une destruction probablement antérieure au dépôt et qui est anthropique. Ils proviennent d'animaux de format moyen comme les ovica- prins ou grand comme les bovins. Le tiers de ces indéterminés, 363 sur 1 170, sont brûlés, de simplement carbonisés à totalement calcinés.

\section{INTERPRÉTATIONS}

\section{Nature des restes}

Les stries et entailles de découpe signent l'origine alimentaire des restes d'ovicaprins et de bovins. Ceux de chien sont plus difficilement interprétables, puisque le squelette entier montre que les cadavres de cette espèce ne sont pas obligatoirement exploités, bien qu'un outil façonné dans un humérus montre qu'elle ne jouit pas d'un statut particulier.

Les restes alimentaires sont apparemment issus de toutes les étapes, de la préparation de la carcasse, comme les bas dc pattcs dc bovins et la région lombo-sacralc d'un ovicaprin, aux " déchets d'assiette ". Les éléments brûlés ne correspondent pas à des traces de cuisson mais à un échauffement des ossements après rejets dans des zones de combustion.

\section{Alimentation}

Elle repose presque uniquement sur les espèces domestiques, mouton, chèvre, porc et bœuf. La part de viande des bovins, évaluée à partir des NMI 
Tabl. VII - Mortalité des bovins d'après les mâchoires : Fs, fosse; S, sondage; P, prémolaire; $M$, molaire; o/-, en éruption; +, ++, +++, slades d'usure croissante ; T, arasée.

\begin{tabular}{|c|c|c|c|c|c|c|c|}
\hline & \multirow{3}{*}{ Fs6 } & \multirow{3}{*}{ décap. } & \multirow{3}{*}{$\frac{\mathbf{S 5}}{1}$} & \multirow{3}{*}{$\frac{\text { Total }}{1}$} \\
\hline & & & & & & & \\
\hline juvénil & & $\mathrm{M} 2 \mathrm{O} /-$ & \pm 18 mois & & & & \\
\hline \multirow{4}{*}{ adulte } & jeune & $\mathrm{P} 4+, \mathrm{M} 3+$ & $2,5<4$ ans & & 1 & & 1 \\
\hline & \multirow{2}{*}{ mature } & $P_{4++}, M 3++$ & $4<8$ ans & & & 1 & 1 \\
\hline & & $\mathrm{P} 4+++, \mathrm{M} 3+++$ & $>8$ ans & 1 & 1 & & 2 \\
\hline & vieux & $\mathrm{P} 4 \mathrm{~T}, \mathrm{M} 3 \mathrm{~T}$ & $\gg 8$ ans & & & & $\mathbf{0}$ \\
\hline \multicolumn{2}{|c|}{ Total NR } & & & 1 & 2 & 2 & 5 \\
\hline
\end{tabular}

Tabl. VIII - Mortalité des borins udultes d'après les épiphysations osseuses : Fs, fosse; $S$, sondage.

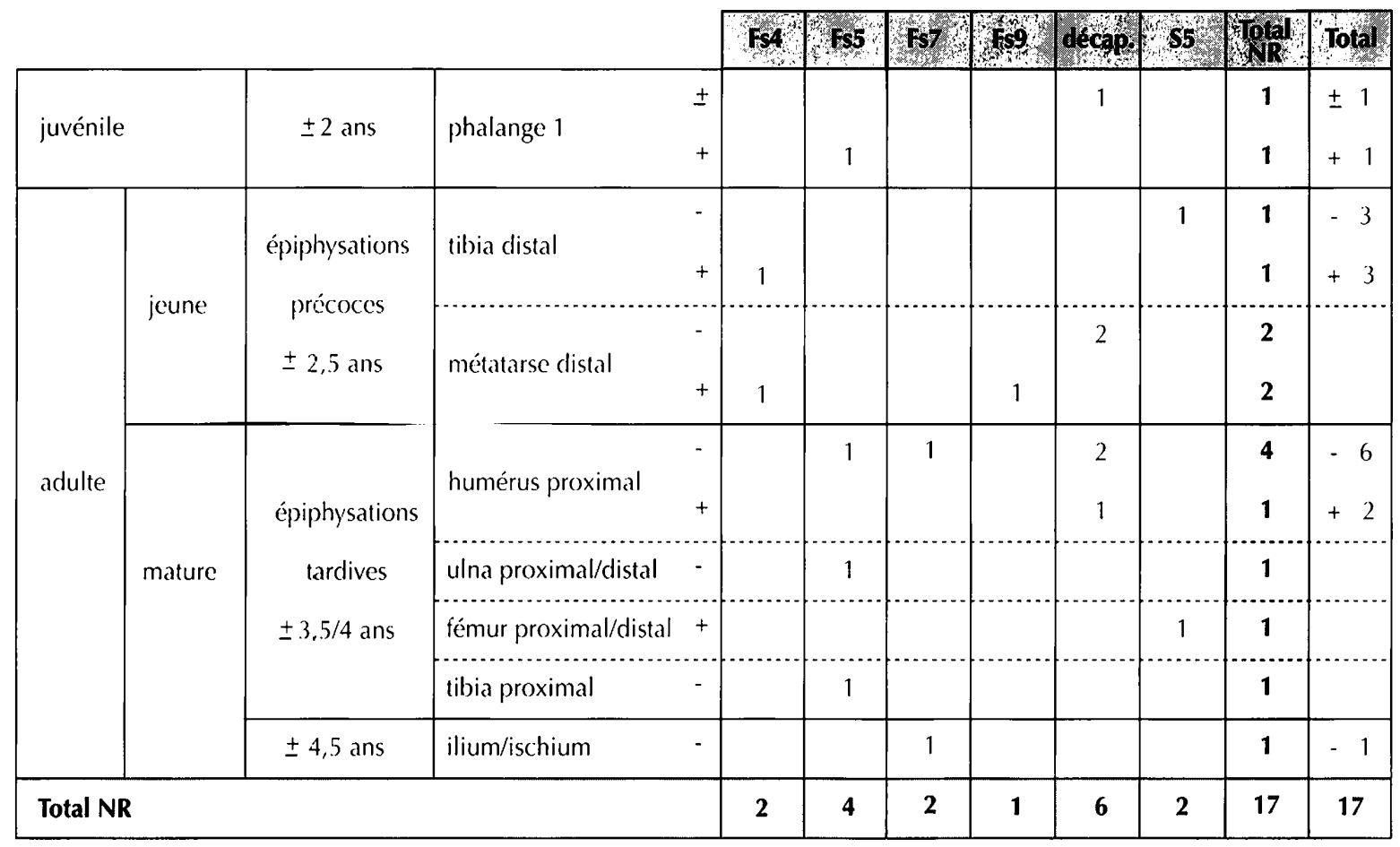

(Columeau, 1991), est très importante, près de $80 \%$, tandis que celle des ovicaprins est faible, quelque $13 \%$ et celle des porcins anecdotique (tabl. II). Aucun âge de consommation ni aucune pièce consommée n'est privilégié.

Ajoutons enfin que les animaux recensés fournissent théoriquement quelque $5400 \mathrm{~kg}$ de viande. Ainsi, par exemple, si une personne mange $200 \mathrm{~g}$ par jour, cette masse a nourri 27000 personnes en un repas ou 74 personnes en une année. Ces valeurs, certes très imprécises, montrent cependant que le lot osseux étudié correspond à une grande quantité de viande consommée.

\section{Élevage}

L'absence de restes sauvages de grand gibier, si l'on considère que les grands bovins sont domestiques ou apparentés, indique que l'approvisionnement carné repose sur les seules espèces domestiques. La consommation d'animaux de tous âges chez les ruminants 
semble signifier que l'élevage devait être pratiqué par les consommateurs eux-mêmes. Aucune gestion spécialisée ne peut être déduite de la mortalité des animaux. Une totale polyvalence potentielle est à retenir pour les ovicaprins, mais, pour les bovins, la relative priorité accordée aux jeunes adultes témoigne d'une consommation d'animaux hors réforme dont la viande offre de bonnes qualités organoleptiques suivant nos critères actuels. Cependant, il n'est pas possible d'affirmer qu'une partie du troupeau est réservée à la production de viande. Les animaux peuvent avoir été choisis parce qu'ils sont estimés inaptes aux services que rend habituellement le troupeau avec des bêtes plus âgées.

La présence marquée d'ovicaprins infantiles et juvéniles aux côtés d'adultes de tous âges, dans la mesure où l'on accepte que leur naissance est saisonnière, signifie que l'occupation du site a duré probablement au moins une année pleine.

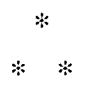

Les lots osseux mis au jour sur le site de La Roquette se caractérisent par un spectre faunique très resserré, dans lequel le lapin érentuellement intrusif est le seul représentant de la faune saurage. Les restes des espèces domestiques se répartissent entre oricaprins (mouton et chèrre) et bovins, ces derniers étant un peu moins nombrcux. Les restes porcins sont rares.

Traduits en données sur l'alimentation, ces dénombrements accordent une part écrasante aux bovins dans l'approvisionnement carné et montrent que l'élevage en est la quasi seule source. Ce dernier se caractérise par une polyvalence des ovicaprins et des bovins.

Ce tableau succinct rejoint ceux dressés à partir des études archéozoologiques sur le Néolithique final (I. Carrère, comm. orale) et sur le Chalcolithique (Forest, 1997) en zone de garrigue du Languedoc oriental. Il s'en rapproche aussi par des particularismes taxinomiques, comme certains orins d'un morphotype probablement différent de ceux des périodes ultérieures et des bovins de grand format au statut encore mal défini.

Le site de Ia Roquette fournit ainsi une nouvelle preuve de l'homogénéité des mobiliers d'origine faunique dans cette région pour cette période, homogénéité qui traduit très probablement une identité de comportement des groupes humains vis-à-vis des animaux et de leur cadavre. 


\section{BIBLIOGRAPHIE}

AMBERT P.

1994 : L'évolution du Languedoc central depuis le Néogène (Crands Causses méridionaux, Piémont languedocien), Documents du Bureau des recherches gcologiques et minières, 231, 210 p., 3 cartes.

BARGE H.

1982 : Les parures du Néolithique ancien au début de l'âge des Métaux en Languedoc, Paris, éd. du CNRS, 396 p., 134 fig., $6 \mathrm{pl}$.

BARONE R.

1976 : Anatomie comparée des mammiferes domestiques, 1-2, 296 et 428 p., Paris, Vigot.

BeAlMe C.

1996 : Le site néolithique final de l'abri de la Vessignée, Saint-Marceld'Ardèche, Ardèche Archéologie, 13, p. 36-44.

BEECHING A.

1980: Introduction ì l'étude des stades néolithique et chalcolithique dans le bassin du Rhône moyen. Quatre fouilles récentes dans leur contexte régional, thèse de Doctorat de $3^{c}$ cycle, univ. LumièresLyon Il, 3 vol.

1995 : Nouveau regard sur le Néolithique ancien et moyen du Bassin rhodanien, in : Voruz J.-L. (éd.), Chronologies néolithiques. De 6000 à 2000 avamt notre ère dans le Bassin thodanien, Actes du colloque d'Ambérieu-en-Bugey, 19-20 sept. 1992, Documents du département d'anthropologie de l'université de Genève, 20, Ambéricu-en-Bugey, éd. Société préhistorique rhodanienne, p. 93-111.

BINDER D., Gilssix B., SÉNFPART I.

1994 : Éléments pour la caractérisation des productions céramiques néolithiques dans le sud de la France, in:
Terre cuite et société. La céramique, document technique, économique, culturel, XIV" Rencontres internationales d'archéologie et d'histoire d'Antibes, Juan-les-Pins, éd. API)CA, p. 255-267.

BOESSNECK].

1969 : Osteological difference between sheep (Ovis aries L.) and goat (Capra hircus L.), in : Brothwell I)., Higgs E. S. (eds), Science in archeology, a survey of progress and research, London, Thames and Hudson, p. 331-358.

BORIRELII. M.

1998 : A propos des faciès du Néolithique final en languedoc oriental : l'hypothèse du groupe des Bruyères, in : D'Anna A., Binder D. (éds), Production et identité culturelle, Actes des rencontres méridionales de préhistoire récente, $2 "$ session, Arles, 8-9 nov. 1996, Juan-les-Pins, éd. APDCA, p. 329-341.

Bol'RciloIs M., BOL'RceOIS R.

1989 : Une grotte sépulcrale à la Bruguière, Bulletin de la Société bagnolaise des sciences historiques et naturelles, 17 , p. $7-15$.

Bitsson-Catil. J.

1995 : Crillon-le-Brave. I a Blaoute, in : Bilan scientifique de la région ProvenceAlpes-Côte d'Azur 1995, Direction régionale des Affaires culturelles, Service régional de l'archéologie, p. 280-282.

Callot: C.

1997 : Diagnose différentielle des principaux éléments squelettiques du lapin igenre (Oryctolagus) et du liezre (genre Lepus) en Europe ocridentale, Fiches d'ostéologie animale pour l'archéologie, série B : Mammifères, $n^{\circ} 3$, Juan-les-Pins, éd. APDCA, 24 p.
Colomer a.

1979 : Les grottes sépulcrales artificielles en I.anguedoc oriental, Toulouse, École. des hautes études en sciences sociales, Archives d'écologie préhistorique, 4,117 p., 48 fig., 30 photos.

Collimbat P.

1991 : L'animal pour l'homme, recherches sur l'alimentation carnée dans le sud do la France du Néolithique au Moyen Âge d'après les vestiges osseux -I- Le monde rural, Travaux du Centre Camille Jullian, 9, univ. AixMarseille 1, Aix-en-Provence, 186 p.

Costantini (:

1984 : Le Néolithique et le Chalcolithique des Grands Causses -IÉtude archéologique, Gallia Préhistoire, 27, 1, p. 121-210.

Collarol J., colks S., Gltherz X., Colomer A.

1980 : La céramique néolithique de l'aven de la Boucle (Corconne, Gard), in: Guilaine J. (éd.), Le groupe de Véraza et la fin des temps néolithiques dans le sud de la France et la Catalogne, Actes du colloque du CNRS, Narbonne, 3-4 juin 1977, Toulouse, éd. du CNRS, p. 200-203.

CuRgiJ.-J.

1965 : Apparition et soudure des points d'ossification des membres chez les mammifêres, Mémoire du Muséum d'histoire naturelle de Paris-Zoologie, 22, 3, p. 17.3-307.

D'AnNa A.

1989 : L'habitat perché néolithique final de la Citadelle (Vauvenargues, Bouches-du-Rhône), in : D'Anna A., Guther\% X. (éds), Enceintes, habitats ceinturés et sites perchés. Du Néolithique au Bronze ancien dans le sud de la France. et les régions voisines, Table ronde de 
Iattes et Aix-en-Provence, 15-18 avr. 1987, Mémoires de la Société languedocienne de préhistoire, 2, Montpellier, éd. de la Société languedocienne de préhistoire, p. 195-208.

1995a : La fin du Néolithique dans le sud-est de la France, in : L'homme méditerranéen, mélanges offerts à Gabriel Camps, Publications de l'univ. d'AixMarseille 1, Laboratoire d'anthropologie et de préhistoire des pays de la Méditerranée occidentale, Aix-enProvence, p. 299-333.

1995b : Le Néolithique final en Provence, in : Voruz. J.-L.. (éd.), Chronologies néolithiques. De 6000 ì 2000 atuant notre ère dans le Bassin rhodanien, Actes du colloque d'Ambérieu-en-Bugey, 19-20 sept. 1992, Documents du département d'anthropologie de l'université de Genève, 20, Ambérieu-en-Bugey, éd. Société préhistorique rhodanienne, p. $265-286$.

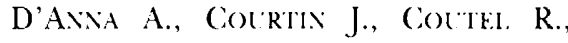
Mulitir A.

1989 : Habitats perchés et enceintes du Véolithique final dans le Lubéron central (Vaucluse), in : D'Anna A., Gutherz X. (éds), Enceintes, habitats ceinturés et sites perchés. Du Néolithique au Bronze ancien dans le sud de la France et les régions voisines, Table ronde de Lattes et Aix-en-Provence, 15-18 avr. 1987, Mémoires de la Société languedocienne de préhistoire, 2, Montpellier, éd. de la Société languedocienne de préhistoire, p. 165-193.

\section{DRAPIER A.}

1995 : Les milieux palustres holocènes de la région de laudun, (sard, mém. de Maîtrise, univ. Paul-Valéry Montpellier III, 36 p., 15 fig., 17 photos.

\section{DRIESCH A. VON DE.N}

1972 : Studien über frühe Tïerknochenfunde von der Iberischen Halbinsel -3Osteoarchäologische Untersuchungen auf der Iberischen Halbinsel, München, Institut für Palaeoanatomie, Domestikationsforschung und Geschichte der Tiermedezin der Universität München.
Écinalimir J-C., JAI.I.OT L.

1992 : Le matériel céramique de MoulinVillard (Caissargues, Gard). Remarques sur la formalisation et l'analyse des données provenant d'observations microscopiques et résultats, Revue d'Archéométrie, 16, p. 71-87.

\section{FOREST V}

1997 : Archéozoologie du Néolithique final au Bronze ancien en Languedoc oriental. Nouvelle synthèse suite aux fouilles préliminaires à linstallation du GazoducArtere du Midi, Rapport interne AFAN-Gazoduc "Artère du midi ", $35 \mathrm{p}$.

\section{Gagitire S., Granitr J.}

1972: Douze ans de recherches archéologiques dans les quartiers nord-ouest d'Avignon, Avignon, publication de la ville, $11 \mathrm{p}$.

\section{GALLAY A.}

1977 : Le Néolithique moyen du Jura et des plaines de la Saône. Contribution à l'étude des relations Chassey-CortaillodMichelsberg, Antiqua, 6, Frauenfelg, éd. Huber, 344 p., 43 fig., 86 pl.

\section{GiscoJ.}

1991 : La chronologie du Néolithique final et du Chalcolithique en Languedoc méditerranéen, in : Le Chalcolithique en Languedoc. Ses relations extra-régionales, Actes du colloque international en hommage au $\mathrm{D}^{\mathrm{r}}$ Jean Arnal, Saint-Matthieu-deTréviers, 20-22 sept. 1990, Archéologie en Languedoc 19901991, p. 217-225.

\section{GEORJON C.}

1997 : Contribution à l'étude du Néolithique final en Languedoc oriental : la Roquette (Tresques, Gard), mém. de DEA, univ. Aix-Marseille 1, Aix-en-Provence, 2 vol., 77 p., 93 fig.

Giligny F., Maréchal D., PÉtrequin P., PÉtreqlix A.-M., SaINTot $S$.

1995 : La séquence néolithique final des lacs de Clairvaux et de Chalain (Jura). Essai sur l'évolution culturelle, in : Voruz J.-L.. (éd.),
Chronologies néolithiques. De 6000 à 2000 arsant notre ère dans le Bassin rhodanien, Actes du colloque d'Ambéricu-en-Bugey, 19-20 sept. 1992, Documents du département d'anthropologie de l'université de Genève, 20, Ambérieu-en-Bugev, éd. Société préhistorique rhodanienne, p. 313-346.

\section{Gillites R.}

1962 : Note sur la découverte d'un vase campaniforme dans le Gard (grotte Canabié-Saint-Victor-la-Coste), Cahiers ligures de préhistoire et d'archéologie, 12, p. 121-165.

1975 : L'habitat du Néolithique final des Bruyères (basse vallée de l'Ardèche), Éludes préhistoriques, 12, p. 1-13.

1988: Le Néolithique el l'Âge du Bronze à la grotte de Saint-Marcel (Ardèche), Ardèche Archéologie, 5, 57 p., 38 fig., 7 annexes, IV photos.

Gros A.-C.

1971 : Compte rendu sommaire sur les fouilles préhistoriques de Serre de Boidon à Grospierres (Ardèche). Campagne de 1970, Les Cahiers $d u$ Grospierrois, Grou Peïro, 4, p. 20-25.

1974 : La grotte du Ranc d'Aven à Grospierres (Ardèche). Campagne de 1970, Les Cahiers du Grospierrois, Grou Peïro, 6, p. 18-27.

Guilaine J., Amifl C., Barthìs P., Collarol J., Vigler J.

1990 : Le Chasséen de l'abri de FontJuvénal, in : Guilaine J., Gutherz. X. (éds), Autour de Jean Arnal, Montpellier, éd. de l'association "Recherches sur les premières communautés paysannes en Méditerranée occidentale ", p. 163-175.

Guil.aine J., Collarou J.

1987 : L'habitat de Roquemengarde à Saint-Pons-de-Mauchiens (Hérault), Études sur l'Hérault, nouv. série, 2, 1986 et 3,1987 , p. 1-10.

GLTherz X.

1975: La culture de Fontbouisse, recherches sur le Chalcolithique en Languedoc orien$t a l$, Publication de l'association pour 
la recherche archéologique en Languedoc oriental, 120 p., 48 fig. 1984 : Les cultures du Néolithique récent et final en Languedoc oriental, thèse de Doctorat de $3^{r}$ cycle, univ. AixMarseille 1, Aix-en-Provence, 4 vol., 345 p., 29 p. de biblio., 247 fig., 9 photos.

\section{GL'THERZ X., COSTE A.}

1974 : Les vases de la grotte de l'Avencas (Brissac, Hérault) et le Néolithique récent dans le Midi de la France, Bulletin de la Sociélé préhistorique fransaise, 71 , ét. et tr. 2, p. 535-542.

GLTHER\% X., JAII.OT L.

1995 : Le Néolithique final du Languedoc méditerranéen, in : Voruz J.-L. (éd.), Chronologies néolithiques. De 6000 à 2000 aveant notre ère dans le Bassin rhodanien, Actes du colloque d'Ambérieu-en-Bugey, 19-20 sept. 1992, Documents du département d'anthropologie de l'université de Geneve, 20, Ambérieu-en-Buger; éd. Société préhistorique rhodanienne, p. 231-263.

Hasler A., (Olllet h., Dirani) (., Chethi.ot P., Richler A., ReNhitit S., Périllato $F$.

1996 : Ventabren "Château-Blane", Rapport de diagnostic TGV ligne :5secteur II : Avignon-Marseille, Service régional de l'archéologie du Languedoc-Roussillon, Association pour les fouilies archéologiques nationales-Méditerranée, SNCF, 241 p., 105 fig.

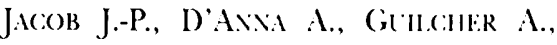
PIGNI M.

1990 : Informations archéologiques, Provence-Alpes-Côte d'Azur, Ciallia Informations, 1 et 2, p. 81-315.

JACOBIESKI $G$.

1988 : La grotte des Menles commune de Rochegude, Gard, mém. de Maîtrise, Paris, univ. Paris I-Sorbonne, 2 vol.

JALIOOI I..

1988 : La station néolithique de Campguivard (Collias, (aard), éléments pour l'étude de la céramique du groupe de Ferrières dans l'Uzège, Archéologie on Languedor, 4, p. 73-82.

\section{Josik: $T$.}

1957 : Le gisement chalcolithique d'Anis -2- Hortus à Valflaunès (Hérault), éude de la faune, Bulletin de la Société préhistorique française, I.IV, 1-2, p. $98-100$.

L.MHERcier O., Blekgik J.F., Dïh P., Loirit D., Melloony P., Pelli.issier M., SERIS D., TCHEREMISSINOFF Y.

1997 : Mondragon "les Juilléras ", Vaucluse, Rapport de fouille TGV ligne : o-secteur II : Valence-Avignon, Service régional de l'archéologie du Languedoc-Roussillon, Association pour les fouilles archéologiques nationales-Méditerranée, SNC.F, 2 rol.

LAMERCIFR O., DUH P., I.OIRAT D., Metiony P., Pellilssier M., Seris D., TCheremissinofF Y., BitgGiR J.-F.

1998 : Les Juilléras (Mondragon, Vaucluse). Site d'habitat et funéraire du Néolithique récentl, Néolithique final, Campaniforme-Bronze ancien et Bronze final 2b : premiers résultats, in : D’Anna A., Binder D. (éds), Production et identité culturelle, Actes des rencontres méridionales de Préhistoire récente, $2^{c}$ session, Arles, 8-9 nov: 1996, Juan-les-Pins, éd. APDCA, p. 359-368.

M.IRGiRIT X., I.OIRIT D., REAMITIT S., T(MH:RF:MISSINOFF Y.

À paraitre: Le Néolithique récent du site de Mondragon/les Ribauds (Vaucluse), in : Le Néolithique sur le TGV Méditerranée, Mémoires d'archéologie méridionale.

MARGiartT X., Rf.NAlitt S.

À paraître : L'établissement Néolithique récent de Mondragon/Le Duc (Vaucluse) : premiers résultats, in : Sociélés et espace, Actes des rencontres méridionales de Préhistoire récente, 3" session, Toulouse, nov. 1998.
Monifet (., L. Mrer N., Ril'x A., Bazil.E. F., DI PIETRo SIRTEN R., (inotong C:

1997 : Rochefort-du-Gard " la Cirange-desMerveilles ", Rapport de diagnostic TGV ligne 5-secteur III : AvignonMontpellier, Service régional de l'archéologie du Languedoc-Roussillon, Association pour les fouilles archéologiques nationales-Méditerranée, SNCF, 2 vol.

MONTJARIIN R.

1970 : Ie gisement néolithique d'Escanin aux Baux-de-Provence, Cahiers rhodaniens, XV, p. 3-152.

1984 : Transition entre le Néolithique final et le Bronze ancien dans trois régions géographiques du Midi de la France : Basse-Ardèche-AlpillesRégion côtière et lagunaire de Sète, in : Waldren W. H., Chapman R., Lewthwaite J., Kennard R. C. (éds) : The Deya Conference of Prehistory. Early setllement in the Western Mediterrariean Islands and their Peripheral Areas, British archaeological reports, series 229, IV, p. 1321-1367.

MOLYON P. HTAL.

A paraître : Le Puits du Fakir à la Chèvre d'Or (Balazuc, Ardèche), Archóologie en Languedoc.

Meliler A., D'ANiNA A.

1986 : Le gisement de plein air chalcolithique de la Plaine-des Blancs à Courthéron, Vaucluse, Bulletin do la Société préhistorique fransaise, 83, 11-12, p. $470-483$.

PIINF S.

$1985:$ Morphological distinctions between the mandibular teeth of young sheep, Oris, and goats, Capra, Journal of Archaeological Science, 12, p. 139-147.

\section{PeitTor H.}

1991 : Plan d'occupation du sol de la commune de Laudun (Gard) de l'epoque préhistorique à l'époque médiénale, mém. de DEA, Montpellier, univ: Montpellier III, $219 \mathrm{p}$.

1992 : Rapport de prospection inventaire programmée "vallée de la Tave", Service 
régional de l'archéologic du I anguedoc-Roussillon, non paginé.

1993 : Rapport de prospection inventaire programmée "vallée de la Tave ", Service régional de l'archéologie du Languedoc-Roussillon, non paginé.

1994 : Rapport de prospection inventaire programmée "vallée de la Tave ", Serrice régional de l'archéologie du Languedoc-Roussillon, non paginé.

A paraître : La Parra-Roquemaure, notice de site, Bulletin de la Société préhistorique francaise.

Pétreglis P., Chastel, J., Gilisily F., Pétrelol's A.-M., Siñtot S.

1988 : Réinterprétation de la civilisation Saône-Rhône : une approche des tendances culturelles du Néolithique final, Gallia Préhistoire, 30, 1987-1988, p. 1-89.

Rat: A., GEORJON C.

1997 : L.e gisement de la Roquette 30330Tresques, Rapport de sauvetage urgent, Service régional de l'archéologie du Languedoc-Roussillon, 27 p., 6 fig., 5 pl., 10 photos.

RAIMOND P.

1900 : L'arrondissement d'tzès avant l'histoire, Paris, Alcan, 263 p.

Roger J.-M., GiTherz X.

1986 : L'habitat néolithique du Mas de Miraman à Nîmes (Gard), Cahiers ligures de préhistoire et de protohistoire, nouv: sćric, 3, p. 209-225.

ROGF.R J.-M., VALETTE P.

1983 : Ia station néolithique final du Bois de Mintau-Calvisson (Gard) (étude préliminairc), Archéologie en Languedoc, 5, 1982-1983, p. 25-38.

ROI:DIL J.-L.

1965 : Le gisement énéolithique de la grotte des Huguenots, Vallon-Pontd'Arc (Ardèche), Gallia Préhistoire, VIII, p. 1-8.
Rotidi. J.-L., SAL MADE: H.

1968 : Ia grotte de Perroche II à Auriolles (Ardèche), Gallia Préhistoire, XI, 1, p. 147-203.

S.ILZ:ADE G.

1983 : Les sépultures du Vaurluse du Néolithique à l'âge du Bronze, ćd. du Laboratoire de paléontologie humaine et de préhistoire, Études quaternaires, mém. 6, 242 p.

Salzade G., Carry A., Chambert A. 1990 : Un nouveau faciès du Néolithique final provençal, le groupe du Fraischamp, l'habitat de La Clairière à La Roque-sur-Pernes (Vaucluse), Gallia Préhistoire, 32, p. 151-178.

S(HIII) E.

1972 : Atlas of Animal Bones, Amsterdam, London, New York, Elsevier Publishing Company, 158 p.

\section{SÉNÉPART I.}

1995 : Chronologie des industries osseuses néolithiques dans le sud-est de la France, in : Voruz J.-L. (éd.), Chronologies néolithiques. De 6000 à 2000 avant notre ère dans le Bassin modanien, Actes du colloque d'Ambérieu-en-Bugey, 19-20 sept. 1992, Documents du département d'anthropologie de l'université de Genève, 20, Ambérieu-en-Bugey, éd. Société préhistorique rhodanienne, p. 205-216.

SILIER I. A.

1969 : The Ageing of Domestic Animals, in : Brothwell D., Higgs E. S. (eds), Science in archeolog;, a survey of progress and research, London, Thames and Hudson, p. 283-302.

St:AREZ C.

1984 : La céramique néolithique de l'abri de Font-Juvénal (Conques, Aude), élude culturelle et spaciale des couches $C 5$ et $C 6$, diplôme de l'École des hautes études en sciences sociales, Toulouse, 151 p., 26 fig.
STRAIM (., THETENOT J.P. (E.DS)

1976 : La civilisation Saône-Rhône, Revue archeologique de l'Est et du cientre-lest, XXVII, 3-4, p. 331-420.

Stlitir M., Pejrson G. W.

1993 : High precision bidecadal calibration of the radiocarbon time scale, $\mathrm{AD} 1950-500 \mathrm{BC}$ and 2500-6000 BC, Radiocarbon, 35, p. 1-23.

VAQLER J.

1975 : La céramique chasséenne du Languedor, Carcassonne, Laboratoire de préhistoire et de palethnologic, 368 p., 78 fig.

1990 : L'évolution du Chasséen méridional, essai dans le bassin de l'Aude, in : Guilaine J., Guther\% X. (éds), Autour de Jean Arnal, Montpellier, éd. de l'association "Recherches sur les premières communautés paysannes en Méditerranée occidentale ", p. $177-189$.

1991 : Aspects du Chasséen en Languedoc occidental. Habitat et culture matériclle, in : Beeching $\mathrm{A}$., Binder D., Blanchet J.-C., Constantin C., Duboulo\% J., Martinez R., Mordant D., Thévenot J.-P., Vaquer J. (éds), Identité du Chasséen, Actes du colloque international de Nemours, 1989 , Nemours, Mémoires du musée de Préhistoire d'Ile-de-France, 4, éd. APRAIF, p. 27-37.

W'olf C.

1991 : Les relations entre les cultures du Midi de la France et de Suisse occidentale au Néolithique rérent et final, in : Le Chalcolithique en Languedoc. Ses relations extra-régionales, Colloque international en hommage au $D^{r}$ Jean Arnal, Saint-Matthieu-deTréviers (Hérault), 20-22 sept. 1990, Archéologie en Languedoc 19901991 , p. $337-346$. 